\title{
AERODYNAMIC DESIGN OF A SUPERCRITICAL CARBON DIOXIDE RADIAL INFLOW TURBINE USING MEANLINE AND COMPUTATIONAL METHODS
}

by

\section{Turner Strang}

A Thesis submitted to the Faculty of Graduate Studies and Research in partial fulfillment of the requirements for the degree of

\section{Master of Applied Science}

in

Aerospace Engineering

Ottawa-Carleton Institute for

Mechanical \& Aerospace Engineering

Department of Mechanical and Aerospace Engineering

Carleton University

Ottawa, Ontario

(c) 2018

Turner Strang 
The undersigned recommend to

the Faculty of Graduate Studies and Postdoctoral Affairs

acceptance of the thesis entitled

\title{
Aerodynamic Design of a Supercritical Carbon Dioxide Radial Inflow Turbine using Meanline and Computational Methods
}

\author{
submitted by \\ Turner Strang \\ in partial fulfillment of the requirements for the degree of \\ Master of Applied Science in Aerospace Engineering
}

Thesis Co-Supervisor:

Professor Henry Saari

Department of Mechanical \& Aerospace Engineering

Thesis Co-Supervisor:

Professor Steen A. Sjolander

Department of Mechanical \& Aerospace Engineering

Department Chair:

Professor Ronald E. Miller

Department of Mechanical \& Aerospace Engineering

Carleton University

2018 


\section{Abstract}

Supercritical Carbon Dioxide (S-CO2) power cycles have gained interest in recent years because they have theoretically been shown to provide higher efficiency than more conventional cycles for heat source temperatures above around $800 \mathrm{~K}$. The main benefit of a S-CO2 power cycles is the low compressor work when it is operated near the critical point, but this provides design challenges. Specifically, properties of S-CO2 vary rapidly, making the computational modelling challenging. The present work presents the aerodynamic design of a radial inflow turbine for a $10 \mathrm{MW}_{\mathrm{e}} \mathrm{S}-\mathrm{CO} 2$ power cycle. A radial in-flow turbine is designed using literature recommendations and then evaluated with computational fluid dynamics (CFD). The turbine off-design performance is characterized with off-design CFD simulations to create performance maps. Overall, the present work details the aerodynamic design of a radial turbine for operation in a $10 \mathrm{MW}_{\mathrm{e}} \mathrm{S}-\mathrm{CO} 2$ power cycle based on meanline and computational methods. 


\section{Acknowledgements}

First, I would like to thank my thesis supervisors, Dr. Henry Saari and Dr. Steen A. Sjolander, for the opportunity to pursue this research, for their guidance, and for their constant support. I am very thankful for the opportunity to work with the both of them.

I would also like to thank Doosan Heavy Industries for funding this work and for being great colleagues to work with.

Finally, I wish to thank my family and friends for their continued support through my many year's of education pursuing my goals. Specifically, I am so grateful for my parents, Jayne and Bruce, for giving me every opportunity I could ever ask for. Also, special thanks to McKenzie for the unconditional support while I chased my dreams. Finally, thanks to my peer and friend, Alberto, for going through this Master's degree journey with me. 


\section{Table of Contents}

$\begin{array}{ll}\text { Abstract } & \text { iii }\end{array}$

Acknowledgments $\quad$ iv

List of Tables viii

List of Figures $\quad x$

List of Symbols $\quad$ Xv

1 Introduction $\quad 1$

2 Literature Review $\quad 5$

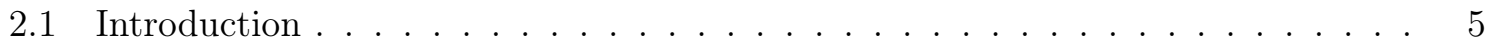

2.2 Supercritical $\mathrm{CO}_{2}$ Power Cycles . . . . . . . . . . . . . . . . . . 5

2.3 Fluid Properties for Supercritical Carbon Dioxide . . . . . . . . . . . . . 8

2.4 Modelling Supercritical Carbon Dioxide Properties . . . . . . . . . . . . . . . . 10

2.5 Turbomachinery Machine Type Selection . . . . . . . . . . . . . . . . . 11

2.6 Radial Inflow Turbine Aerodynamic Design . . . . . . . . . . . . . . . . . . 14

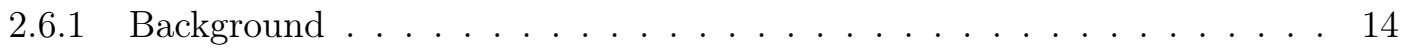

2.6 .2 CFD Analysis . . . . . . . . . . . . . . . . . . . . 24

2.6.3 Radial Turbine Performance Characteristics . . . . . . . . . . . . . . . 26

3 Radial Turbine One-Dimensional Development 28

3.1 Introduction . . . . . . . . . . . . . . . . . . . . 28 
3.2 One-Dimensional Design Methodology . . . . . . . . . . . . . . . . . . 29

3.2.1 Rotor One-Dimensional Design Methods . . . . . . . . . . . . 30

3.2.2 Nozzle One-Dimensional Design Methods . . . . . . . . . . . . . 38

3.2.3 Volute One-Dimensional Design Methods . . . . . . . . . . . . . 43

$3.3 \mathrm{~S}^{-\mathrm{CO}_{2}}$ Radial Turbine One-Dimensional Design . . . . . . . . . . . . . . 46

3.3 .1 Turbine Design Specifications . . . . . . . . . . . . . . 46

3.3.2 Rotor One-Dimensional Design . . . . . . . . . . . . . . . 47

3.3.3 Stator One-Dimensional Design _. . . . . . . . . . . . 51

4 Computational Turbine Aerodynamic Development 53

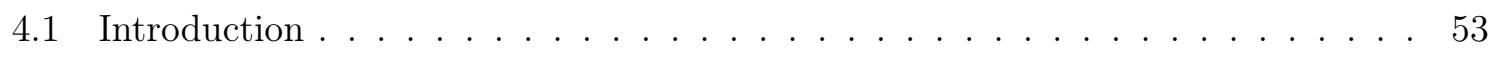

4.2 Development of Three-Dimensional Components . . . . . . . . . . . . 53

4.2.1 Rotor Three-Dimensional Implementation . . . . . . . . . . . . . 54

4.2 .2 Stator Three-Dimensional Implementation . . . . . . . . . . . . . 56

4.3 CFD Background . . . . . . . . . . . . . . . . . . 61 61

4.4 Computational Volute Development . . . . . . . . . . . . . 65

4.5 Computational Rotor Development . . . . . . . . . . . . . . . . 69

4.5 .1 Tip Clearance . . . . . . . . . . . . . . . 70

4.5 .2 Wrap Angle . . . . . . . . . . . . . . . . . . 71

4.5.3 Hub and Shroud Meridional Profile . . . . . . . . . . . . . 72

4.6 Computational Nozzle and Rotor Development . . . . . . . . . . . . 73

4.6 .1 Nozzle Metal Angle Adjustment . . . . . . . . . . . . . . . . . 74

4.6 .2 Rotor Metal Angle Adjustment . . . . . . . . . . . . . . . . 77

4.7 On-Design Results . . . . . . . . . . . . . . . . . . 79

4.8 Importance of Real Gas Properties Implementation . . . . . . . . . . . . . . 84

5 Off-Design Modelling $\quad 89$

5.1 Introduction . . . . . . . . . . . . . . . . . 89

5.2 Turbine Performance Parameters and Characteristics . . . . . . . . . . . . 89

5.3 Off-Design Modelling Methods . . . . . . . . . . . . . . . 91 
5.4 Results . . . . . . . . . . . . . . . . . . . . . . . 92

6 Summary, Conclusions, and Recommendations 99

6.1 Summary . . . . . . . . . . . . . . . . . . . . . . . 99

6.2 Conclusions and Recommendations . . . . . . . . . . . . . . . . . 100

$\begin{array}{ll}\text { List of References } & 103\end{array}$

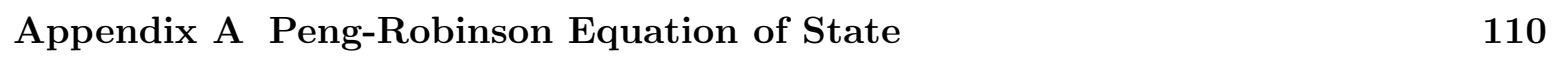

Appendix B Aungier's Rotor Meridional Profiles 114

$\begin{array}{lll}\text { Appendix C Computational Mesh Detail } & 117\end{array}$

Appendix D RGP Table Generation 122

D.1 Script from ANSYS . . . . . . . . . . . . . . . . . . 122

D.2 RGP Tables . . . . . . . . . . . . . . . . . . . . . . . 124

$\begin{array}{lll}\text { Appendix E } & \text { Performance Map Curve Fit Methods } & 128\end{array}$ 


\section{List of Tables}

2.1 S-CO2 turbine machine type corresponding to various proposed and existing plants with a range of output powers (Data obtained from Allison et al., 2017). 13

3.1 Turbine thermodynamic design inputs. . . . . . . . . . . . . . . . . 46

3.2 Rotor variable design inputs. . . . . . . . . . . . . . . . . . . 49

3.3 Rotor one-dimensional design output parameters. . . . . . . . . . . . . . . . 50

3.4 Nozzle one-dimensional design output parameters. . . . . . . . . . . . . . . . 52

4.1 Bezier curve control points for default radial turbine meridional profile in Blade-

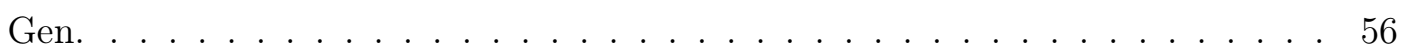

4.2 Volute, nozzle, and rotor wall $y^{+}$values. . . . . . . . . . . . . . 64

4.3 Percent change of volute pressure loss coefficient for different volute inlet turbulence cases. . . . . . . . . . . . . . . . . . . 67

4.4 Effect of volute Swirl Coefficient on nozzle inlet flow angle. . . . . . . . . . . . . 69

4.5 Rotor efficiency for BladeGen default and Aungier meridional profiles. . . . . . 73

4.6 Property evaluation locations offset from blade leading and trailing edges. . . . 74

4.7 Rotor inlet flow property comparison between the design case and the CFD results after the volute and nozzles with design and adjusted nozzle trailing edge metal angles. . . . . . . . . . . . . . . . . 76

4.8 Rotor outlet flow property comparison between the design case and the CFD results with design and adjusted rotor trailing edge metal angles. . . . . . . . 78

4.9 Comparison of final steady-state CFD results with design case in terms of thermodynamic state points and performance. . . . . . . . . . . 80 
4.10 Comparison of CFD results with ideal gas and real gas properties in terms of thermodynamic state points and performance. . . . . . . . . . . 87

5.1 Percent change of average total properties and change in flow angle at volute outlet (Station 2) for different mass flow rates with the design value $(170 \mathrm{~kg} / \mathrm{s})$ as the baseline. . . . . . . . . . . . . . . . . . . . . . . . 92

5.2 Property evaluation locations offset from blade leading and trailing edges. . . . 94

A.1 Zero-pressure specific heat coefficients for $\mathrm{CO}_{2}$ related to Equation A.6 (Obtained from Poling et al., 2001). . . . . . . . . . . . . . . . . . . . . . . . 112

A.2 Pressure and temperature values of the 81 state points used for comparison of Peng-Robinson and REFPROP property calculation. . . . . . . . . . . . . 113

A.3 Statistics for percentage deviation of calculated enthalpy, entropy, and density values using the presented Peng-Robinson implementation compared to REF-

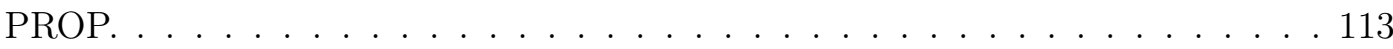

D.1 Dependent variable for the nine RGP tables. . . . . . . . . . . . . . . . . . . 124 


\section{List of Figures}

1.1 Cycle efficiency versus turbine inlet temperature (Dostal, 2004) . . . . . . . . . 2

1.2 Example regenerative Brayton cycle schematic and $T-s$ diagram. . . . . . . 2

1.3 Variation of density and specific heat capacity with temperature and pressure for $\mathrm{CO}_{2}$ (Friedman \& Anderson, 2017) . . . . . . . . . . . . 3

2.1 Phases of $\mathrm{CO}_{2}$ with respect to pressure and temperature (Adapted from Finney \& Jacobs, 2010). (Critical values obtained from Span \& Wagner (1996).) . . . . 6

2.2 CUBCL simple regenerative Brayton cycle (Adapted from MacLean, 2012, by Alberto J. Martel Matos, Carleton University). . . . . . . . . . . . . . . . 7

2.3 CUBCL simple regenerative Brayton cycle represented on a temperature versus

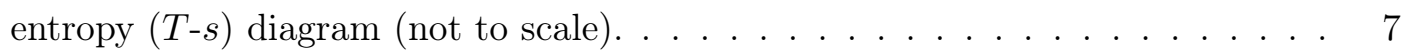

2.4 Compressibility factor versus reduced pressure and reduced temperature with example S-CO2 turbomachinery inlet and outlet points (Adapted from Sjolan-

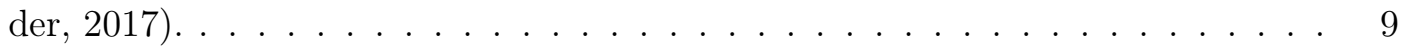

2.5 Specific speed chart for preliminary turbomachine type selection (Adapted from Csanady (1964)by Sjolander (2017)) . . . . . . . . . . . . . . . . . 12

2.6 Radial turbine components and station locations for sizing. . . . . . . . . . . 15

2.7 General $h-s$ diagram for the expansion process through a turbine with state points corresponding to Figure 2.6 (White, 2015) . . . . . . . . . . . . 16

2.8 Radial turbine rotor sizing dimensions. . . . . . . . . . . . . . . . . . . . . . . . 19

2.9 Radial turbine rotor example velocity diagrams. . . . . . . . . . . . . . . . . 20

2.10 Work Coefficient versus Flow Coefficient with lines of constant total-static efficiency (Japikse \& Baines, 1994). . . . . . . . . . . . . . . . . . . . . . . 21 
2.11 General radial turbine nozzle dimensions. . . . . . . . . . . . . . . . . . 23

2.12 General radial turbine scroll volute dimensions. . . . . . . . . . . . . . . . . 23

2.13 An example turbine map plotting the turbine's performance in terms of pressure ratio versus the mass flow parameter (Adapted from Sjolander, 2017). . . . . . 27

3.1 Radial turbine components and station locations for sizing. . . . . . . . . . . 30

3.2 Radial turbine rotor sizing dimensions. . . . . . . . . . . . . . . . . . . . . 31

3.3 Radial turbine rotor example velocity diagrams. . . . . . . . . . . . . . . . . 31

3.4 Rotor inlet velocity triangle. . . . . . . . . . . . . . . . . . . . . . 32

3.5 Rotor outlet velocity triangle. . . . . . . . . . . . . . . . . . . . 35

3.6 Illustration for nozzle throat dimensions (Adapted from Aungier, 2006). . . . . 41

3.7 Illustration of nozzle chord in relation to nozzle inlet radii, outlet radii, and outlet setting angle. . . . . . . . . . . . . . . . . . . . . 42

3.8 General radial turbine scroll volute dimensions. . . . . . . . . . . . . . . . . . . 44

3.9 Variation in $\xi$ with $\Psi$ and $\Phi$ at different $\beta_{4}$ values. . . . . . . . . . . . . 48

3.10 Variation in $\phi_{R}$ with $\Psi$ and $\Phi . \ldots \ldots \ldots$. . . . . . . . . . . . . 49

3.11 One-dimensional rotor meridional geometry (Dimensions in $\mathrm{mm}$ ). . . . . . . . 50

3.12 One-dimensional rotor velocity triangles (rotor geometry not to scale). . . . . . 51

3.13 One-dimensional volute and rotor geometry (Dimensions in $\mathrm{mm}$ ) . . . . . . . . . 51

4.1 Rotor blade thickness distribution. . . . . . . . . . . . . . . . . . . 54

4.2 Illustration of trailing edge wrap angle. . . . . . . . . . . . . . . . . 55

4.3 Rotor blade metal angle, $\beta^{\prime}$, and wrap angle, $\theta^{\prime}$, distribution for trailing edge wrap angles, $\theta_{t e}^{\prime}$, of $40^{\circ}, 60^{\circ}$, and $80^{\circ} \ldots \ldots \ldots \ldots \ldots \ldots$

4.4 Comparison of BladeGen default and Aungier meridional profiles. . . . . . . . . 59

4.5 Turbine rotor in three-dimensions. . . . . . . . . . . . . . . . . 59

4.6 Nozzle blade angle and thickness distributions. . . . . . . . . . . . . . . . . 60

4.7 Turbine with volute, nozzles, and rotor. . . . . . . . . . . . . . 60

4.8 Mesh independence studies for rotor. . . . . . . . . . . . . . . . . . . . 63

4.9 Mesh independence study for nozzles. . . . . . . . . . . . . . . . . . 63

4.10 Mesh independence study for volute. . . . . . . . . . . . . . . . . 63 
4.11 Rotor flat plate Reynolds number versus meridional distance to locate laminarturbulent transition. . . . . . . . . . . . . . . . . 65

4.12 Volute domain without and with radial extension at outlet. . . . . . . . . . 66

4.13 Turbulence eddy dissipation through a representative cross-section of the volute $(z=7.1 \mathrm{~mm})$ for the case where Medium Intensity is specified at the volute inlet. 67

4.14 Turbulence eddy dissipation through a representative cross-section of the volute $(z=7.1 \mathrm{~mm})$ for the case where $k=0.2 \mathrm{~m}^{2} / \mathrm{s}^{2}$ and $\epsilon=500 \mathrm{~m}^{2} / \mathrm{s}^{3}$ are specified at the volute inlet. . . . . . . . . . . . . . . . . . . 68

4.15 Example variation of flow around periphery of volute outlet (Adapted from Japikse \& Baines, 1994) . . . . . . . . . . . . . . . . . . . . 68

4.16 Variation of flow around periphery of volute outlet with $S_{c}=0.85$. (Tongue location is $40^{\circ} \mathrm{CCW}$ from the station 1 plane.) . . . . . . . . . . . . 69

4.17 Variation of flow around periphery of volute outlet with $S_{c}=0.95$. (Tongue location is $40^{\circ} \mathrm{CCW}$ from the station 1 plane.) . . . . . . . . . . . 69

4.18 Change in rotor efficiency versus tip clearance gap. . . . . . . . . . . . . . . 71

4.19 Change in rotor efficiency versus trailing edge wrap angle. . . . . . . . . . . . . 72

4.20 Comparison of Mach number distribution on meridional plane for BladeGen default and Aungier meridional profiles. . . . . . . . . . . . . . . . 73

4.21 Nozzle blade loading at $50 \%$ span. . . . . . . . . . . . . . . . . 75

4.22 Mach number and velocity vectors through nozzle row at $50 \%$ span. . . . . . . 75

4.23 Comparison of rotor inlet velocity triangle design case and CFD results with design and adjusted nozzle metal angles. . . . . . . . . . . . . . . . . 76

4.24 Comparison of rotor outlet velocity triangle design case and CFD results with design and adjusted rotor trailing edge metal angles. . . . . . . . . . . . 78

4.25 Mach number and velocity vectors through nozzles and rotor at $50 \%$ span. . . . 82

4.26 Rotor blade loading at $20 \%, 50 \%$, and $80 \%$ span. . . . . . . . . . 83

4.27 Percent error of $\rho$ for the ideal gas implementation versus REFPROP plotted against $P$ and T. ([REFPROP Value - Ideal Gas Value]/REFPROP Value.) . . 85 
4.28 Percent error of $c_{\mathrm{p}}$ for the ideal gas implementation versus REFPROP plotted against $P$ and T. ([REFPROP Value - Ideal Gas Value]/REFPROP Value.) . . 85

4.29 Comparison of rotor inlet velocity triangle CFD results with ideal gas and real gas properties. . . . . . . . . . . . . . . . . 86

4.30 Comparison of rotor outlet velocity triangle design CFD results with ideal gas and real gas properties. . . . . . . . . . . . . . . . 87

5.1 Flow Parameter versus Enthalpy Drop Parameter for Speed Parameters of $80 \%$, $100 \%$, and $120 \%$ of the design speed. . . . . . . . . . . . . . 93

5.2 Total-total Isentropic Efficiency versus Enthalpy Drop Parameter for Speed Parameters of $80 \%, 100 \%$, and $120 \%$ of the design speed. . . . . . . . . . 94

5.3 Average Mach number at critical locations versus Enthalpy Drop Parameter for Speed Parameters of $80 \%, 100 \%$, and $120 \%$ of the design speed. . . . . . . 95

5.4 Mach number and velocity vectors through nozzles and rotor at $50 \%$ span for choking Flow Parameters and Speed Parameters of $80 \%$ and $120 \%$ of the design

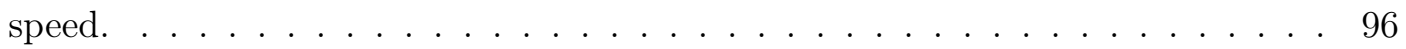

5.5 Rotor inlet relative flow angle versus Flow Parameter for Speed Parameters of $80 \%, 100 \%$, and $120 \%$ of the design speed. . . . . . . . . . . . 97

5.6 Mach number and velocity vectors through nozzles and rotor at $50 \%$ span for a Flow Parameter of $1.88 \times 10^{-2}$ and design Speed Parameter. . . . . . . . . 97

B.1 Circular-arc hub contour (Adapted from Aungier, 2006) . . . . . . . . . . . . 115

B.2 Power-law shroud contour (Adapted from Aungier, 2006) . . . . . . . . . . 116

B.3 Variation in shroud contour with $n$ values. . . . . . . . . . . . . . 116

C.1 Volute mesh detail. . . . . . . . . . . . . . . . . . . . . . . 118

C.2 Nozzle mesh detail. . . . . . . . . . . . . . . . . . . . . . . . . . . . . 119

C.3 Rotor mesh detail. . . . . . . . . . . . . . . . . . . . . . . . . 121

D.1 Pressure and temperature grids of Table 1 of RGP file used for the present work.125

D.2 Pressure and temperature grids of Table 2 of RGP file used for the present work.125

D.3 Pressure and temperature grids of Table 3 of RGP file used for the present work.125

D.4 Pressure and temperature grids of Table 4 of RGP file used for the present work.126 
D.5 Pressure and temperature grids of Table 5 of RGP file used for the present work.126

D.6 Pressure and temperature grids of Table 6 of RGP file used for the present work.126

D.7 Pressure and temperature grids of Table 7 of RGP file used for the present work.127

D.8 Pressure and temperature grids of Table 8 of RGP file used for the present work.127

D.9 Pressure and temperature grids of Table 9 of RGP file used for the present work.127

E.1 Flow Parameter versus Enthalpy Drop Parameter for Speed Parameters of 80\%, $100 \%$, and $120 \%$ of the design speed with curve fit relationship from White (2015).129

E.2 Flow Parameter versus Enthalpy Drop Parameter for Speed Parameters of 80\%, $100 \%$, and $120 \%$ of the design speed with improved curve fit relationship. . . . 129

E.3 Total-total Isentropic Efficiency versus Enthalpy Drop Parameter for Speed Parameters of $80 \%, 100 \%$, and $120 \%$ of the design speed. . . . . . . . 130 


\section{List of Symbols}

\begin{tabular}{ll}
\hline Symbols & Definition \\
\hline$A$ & Area $\left[\mathrm{m}^{2}\right]$ \\
$A^{\prime}$ & Volute cross section ellipse axial dimension \\
$R$ & Aspect ratio \\
$a$ & Speed of sound $[\mathrm{m} / \mathrm{s}]$ \\
$B^{\prime}$ & Volute cross section ellipse radial dimension \\
$b$ & Blade height $[\mathrm{m}]$ \\
$C_{N}$ & Velocity $[\mathrm{m} / \mathrm{s}]$ \\
$C_{s}$ & Spouting velocity $[\mathrm{m} / \mathrm{s}]$ \\
$c_{\mathrm{p}}$ & Specific heat capacity at constant pressure $[\mathrm{kJ} / \mathrm{kgK}]$ \\
$c_{\mathrm{v}}$ & Specific heat capacity at constant temperature $[\mathrm{kJ} / \mathrm{kgK}]$ \\
& Nozzle chord $[\mathrm{m}]$ \\
& Diameter $[\mathrm{m}]$ \\
&
\end{tabular}




\begin{tabular}{|c|c|}
\hline$\Delta H$ & Head rise \\
\hline$i$ & Incidence $\left[{ }^{\circ}\right]$ \\
\hline$K_{N}$ & Nozzle total pressure loss coefficient \\
\hline$K_{V}$ & Volute total pressure loss coefficient \\
\hline$k$ & Turbulent kinetic energy $\left[\mathrm{m}^{2} / \mathrm{s}^{2}\right]$ \\
\hline$L_{R}$ & Axial length of rotor \\
\hline Ma & Mach number \\
\hline$\dot{m}$ & Mass flow rate $[\mathrm{kg} / \mathrm{s}]$ \\
\hline$N$ & Rotational speed [rpm] \\
\hline$N_{N}$ & Nozzle blade count \\
\hline$N_{R}$ & Rotor blade count \\
\hline$N_{s}$ & Specific speed \\
\hline$n$ & Isentropic exponent \\
\hline$o_{t h}$ & Throat opening [mm] \\
\hline$P$ & Pressure $[\mathrm{Pa}]$ \\
\hline$P_{R}$ & Reduced pressure \\
\hline$Q$ & Volumetric flow rate $\left[\mathrm{m}^{3} / \mathrm{s}\right]$ \\
\hline$R$ & Specific gas constant $[\mathrm{J} / \mathrm{kgK}]$ \\
\hline$R$ & Stage reaction \\
\hline$R e$ & Reynolds Number \\
\hline
\end{tabular}




\begin{tabular}{|c|c|}
\hline $\operatorname{Re}_{M}$ & Machine Reynolds number \\
\hline$R e_{F P}$ & Flat plate Reynolds number \\
\hline$R P M$ & Rotational velocity [rpm] \\
\hline$r$ & Radius $[\mathrm{m}]$ \\
\hline$S_{c}$ & Swirl Coefficient \\
\hline$s$ & Entropy $[\mathrm{kJ} / \mathrm{K}]$ \\
\hline$s$ & Blade pitch $[\mathrm{m}]$ \\
\hline$T$ & Temperature $\left[\mathrm{K}\right.$ or $\left.{ }^{\circ} \mathrm{C}\right]$ \\
\hline$T$ & Torque $[\mathrm{kJ}]$ \\
\hline$T_{R}$ & Reduced temperature \\
\hline$t_{b}$ & Blade thickness $[\mathrm{m}]$ \\
\hline$U$ & Blade speed $[\mathrm{m} / \mathrm{s}]$ \\
\hline$W$ & Relative velocity $[\mathrm{m} / \mathrm{s}]$ \\
\hline$\dot{W}$ & Power $[\mathrm{W}]$ \\
\hline$y^{+}$ & Non-dimensional distance from the wall \\
\hline$Z$ & Compressibility factor \\
\hline$z$ & Axial distance $[\mathrm{m}]$ \\
\hline
\end{tabular}

\section{Greek}

$\begin{array}{ll}\alpha & \text { Absolute flow angle }\left[^{\circ}\right] \\ \beta & \text { Relative flow angle }\left[{ }^{\circ}\right]\end{array}$




\begin{tabular}{|c|c|}
\hline$\beta^{\prime}$ & Blade metal angle $\left[^{\circ}\right]$ \\
\hline$\eta$ & Isentropic efficiency \\
\hline$\epsilon$ & Rotor inlet-outlet radius ratio \\
\hline$\epsilon$ & Turbulent eddy dissipation $\left[\mathrm{m}^{2} / \mathrm{s}^{2}\right]$ \\
\hline$\gamma$ & Specific heat ratio \\
\hline$\Omega$ & Specific speed \\
\hline$\omega$ & Angular velocity $[\mathrm{rad} / \mathrm{s}]$ \\
\hline$\Phi$ & Flow Coefficient \\
\hline$\phi_{R}$ & Rotor Relative Velocity Coefficient \\
\hline$\Psi$ & Work Coefficient \\
\hline$\mu_{4}$ & Dynamic viscosity [Pas] \\
\hline$\nu$ & Kinematic viscosity $\left[\mathrm{m}^{2} / \mathrm{s}\right]$ \\
\hline$\nu_{t s}$ & Isentropic velocity ratio \\
\hline$\tau_{\text {wall }}$ & Wall shear stress \\
\hline$\theta^{\prime}$ & Camberline (wrap) angle \\
\hline$\xi$ & Meridional Velocity Ratio \\
\hline
\end{tabular}

\section{Subscript}

Total (stagnation) conditions

Turbine inlet (volute inlet) state point

Nozzle inlet state point 
3

4

5

$c$

e

$m$

$\max$

$N$

$R$

ref

rel

shaft

$s$

ss

$t$

$t$

te

th

ts

$t t$
Nozzle outlet state point

Rotor inlet state point

Rotor outlet state point

Critical condition

Electric power output

Meridional component of velocity

Maximum value

Nozzle

Rotor

Reference state

Relative to rotating frame

Shaft output power

Shroud location

Isentropic condition

Tip location

Turbine output power

Trailing edge

Throat location

Total-static

Total-total 
V

$\theta$

Acronyms

AT

CFD

$\mathrm{CO}_{2}$

DAT

EoS

RT

RGP

$\mathrm{S}-\mathrm{CO} 2$
Volute

Tangential component of velocity

Axial turbine

Computational fluid dynamics

Carbon dioxide

Double-flow axial turbine

Equation of state

Radial turbine

Real gas properties

Supercritical $\mathrm{CO}_{2}$ 


\section{Chapter 1}

\section{Introduction}

Alternative power generation methods have been gaining interest due to the economic and environmental cost of current methods. Supercritical $\mathrm{CO}_{2}(\mathrm{~S}-\mathrm{CO} 2)$ power cycles provide an alternative to more conventional power generation techniques such as steam power cycles. S$\mathrm{CO} 2$ power cycles are favourable in some applications because they have been shown to have higher theoretical efficiency for certain heat source temperatures as shown in Figure 1.1. This higher efficiency power generation would that for the same power requirements, less fuel has to be consumed. Using less fuel would reduce the economic and environmental cost of power generation. Also, at usual operating conditions, S-CO2 has a higher density than steam, allowing for much more smaller turbomachinery (Dostal, 2004). These benefits have been the basis for research into the applicability of S-CO2 power cycles for various applications. As discussed by Musgrove \& Wright (2017), these applications include waste heat recovery, concentrated solar power, nuclear, and others.

The variety in applications of S-CO2 power cycles has led to a wide variety of cycle configurations. Different applications have different source temperatures and different optimization strategies as discussed by Crespi et al. (2017). Some cycles are optimized for maximum output power whereas others are optimized for maximum cycle efficiency, but they all operate on the

basic thermodynamics of converting input heat into output mechanical power. S-CO2 power cycles may be provided heat directly through combustion or indirectly through heat exchangers. The simplest example of an indirect $\mathrm{S}-\mathrm{CO} 2$ power cycle is the recuperated Brayton cycle as shown in Figure 1.2. Heat is added to the $\mathrm{CO}_{2}$ through the main heat exchanger, then it 


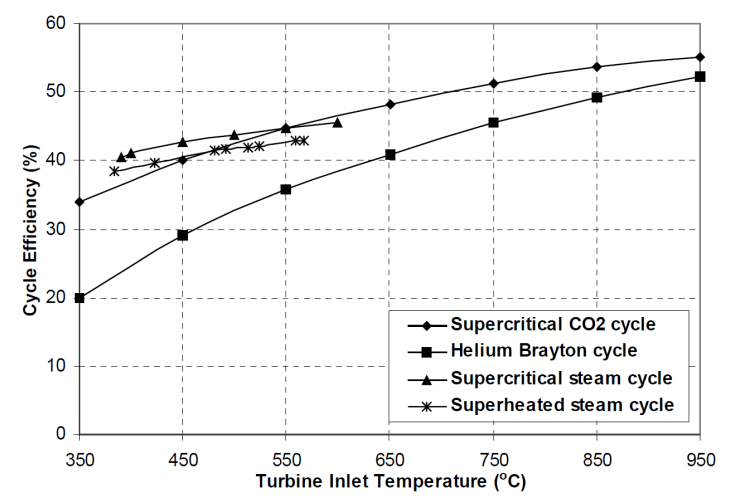

Figure 1.1: Cycle efficiency versus turbine inlet temperature (Dostal, 2004).

enters the turbine where the rotor converts the energy in the fluid into mechanical energy in an output shaft connected to a generator to generate electricity. The fluid leaving the turbine goes through a recuperator to transfer heat to the fluid leaving the compressor. After the recuperator, at point 3, a precooler cools the fluid to the desired compressor inlet temperature which is typically close to the fluid's critical point. Then, the compressor does work on the fluid to reach the required turbine inlet pressure and the recuperator and main heat exchanger raise the fluid temperature to the specified turbine inlet temperature.

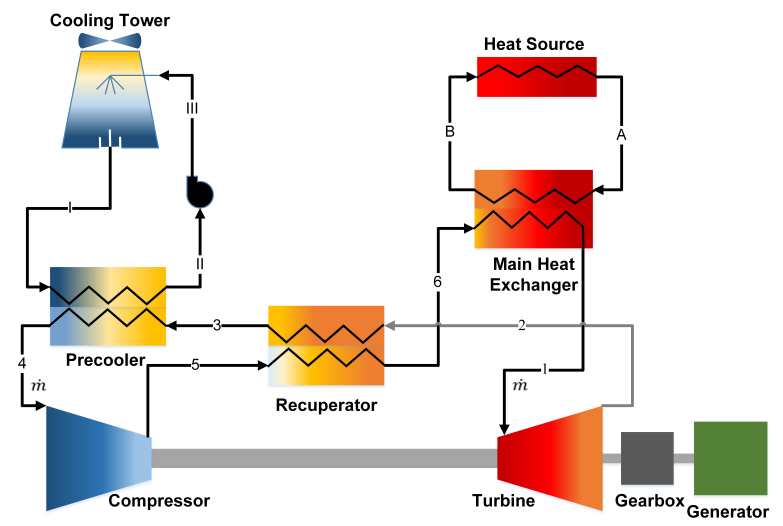

(a) Cycle schematic (Adapted from MacLean, 2012, by Alberto J. Martel Matos, Carleton University).

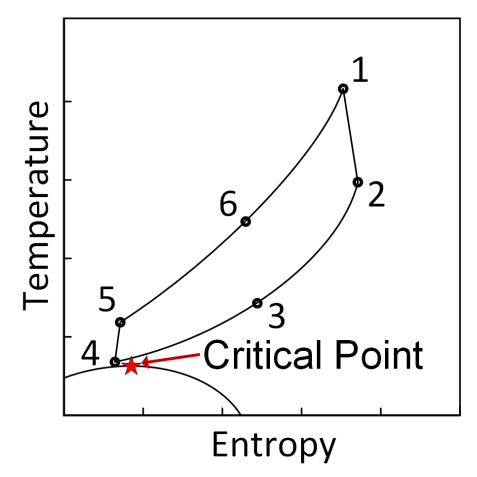

(b) Temperature-entropy diagram.

Figure 1.2: Example regenerative Brayton cycle schematic and $T-s$ diagram.

S-CO2 exhibits real gas properties with large variations in properties such as density and specific heat capacity with small changes in pressure and temperature near the critical point. These variations are shown in Figure 1.3 where the critical point of $\mathrm{CO}_{2}$ is at $7.377 \mathrm{MPa}$ and 
$304.13 \mathrm{~K}\left(30.98^{\circ} \mathrm{C}\right)$ (Span \& Wagner, 1996). The real gas properties of S-CO2 subject the compressor to a large variation in fluid properties through the compression process because it is beneficial to operate it close to the critical point of the fluid to minimize the required compression work. The turbine is affected by real gas properties to a lesser extent because its operating point is usually further from the critical point, but it is just as important to take real gas properties into consideration in the design phase.

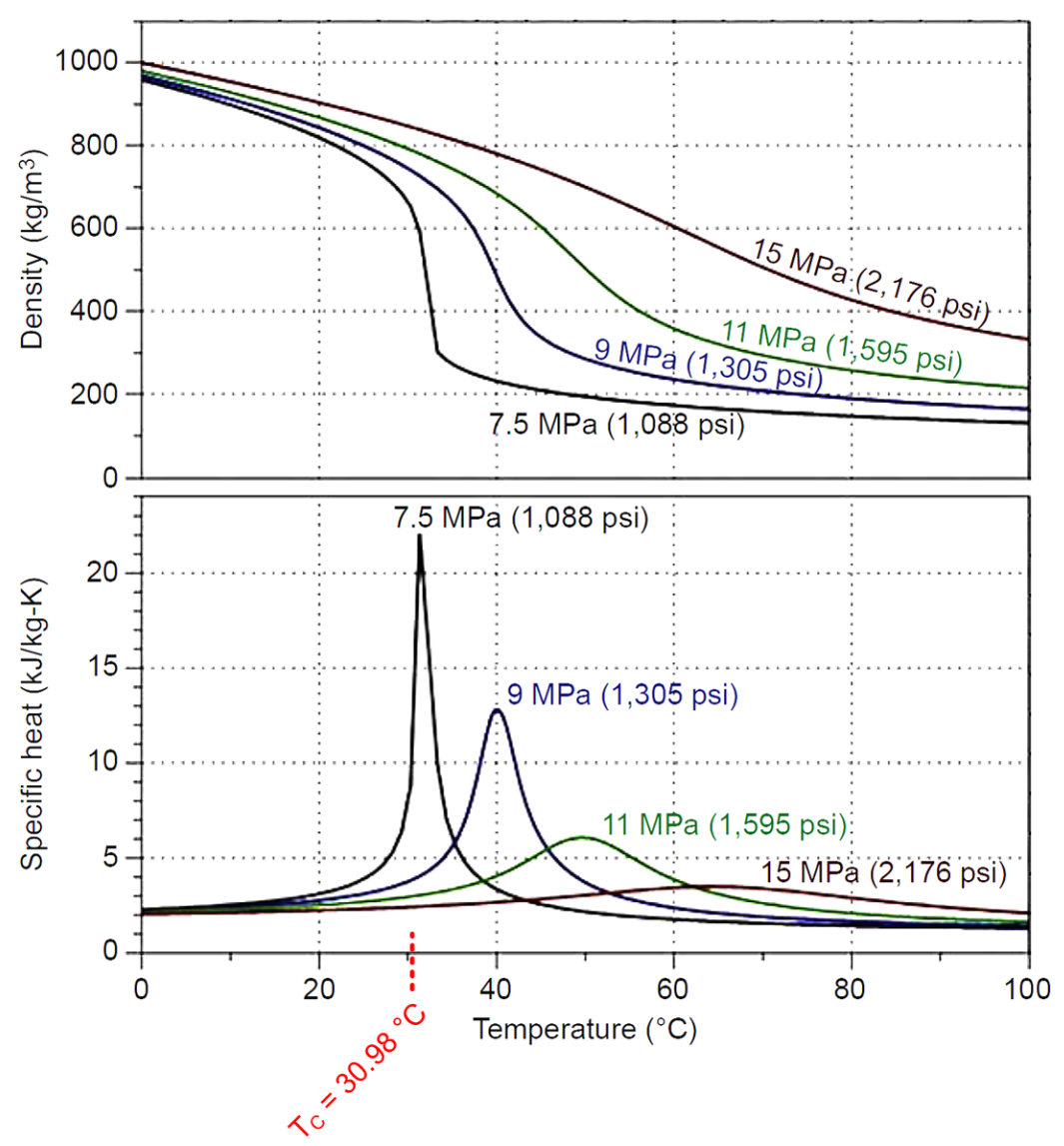

Figure 1.3: Variation of density and specific heat capacity with temperature and pressure for $\mathrm{CO}_{2}$ (Friedman \& Anderson, 2017).

The aim of this project was to design the turbine for a $10 \mathrm{MW}_{\mathrm{e}} \mathrm{S}-\mathrm{CO} 2$ power cycle as part of a team investigating the feasibility of a $10 \mathrm{MW}_{\mathrm{e}} \mathrm{S}-\mathrm{CO} 2$ power cycle for a waste heat recovery application. The research was funded by Doosan Heavy Industries (DHI). Carleton University's Brayton Cycle Loop (CUBCL) 4th year undergraduate Capstone Project has provided a solid knowledge base for S-CO2 power cycles at the university. DHI performed 
the cycle optimization, determined the operating point for the turbine, and specified that the turbine would be a radial inflow type based on their preliminary analysis. The turbine was to be designed and evaluated to meet the required output power at an acceptable efficiency and then the off-design performance was to be characterized for use in the dynamic modelling of the project for control system definition.

The scope of this thesis is the aerodynamic design of a radial inflow turbine as the power generating component of an S-CO2 power cycle. A review of literature for this topic has been provided in Chapter 2. Radial inflow turbine design literature is generally based on ideal gas assumptions, so the design of a real gas radial turbine is not trivial. The preliminary design using literature recommendations with adaptations to account for real gas properties is covered in Chapter 3. Chapter 4 covers the evaluation and improvement of the turbine design at the design operating point using Computational Fluid Dynamics (CFD). Finally, Chapter 5 covers the development of the turbine performance maps to characterize the offdesign performance of the designed machine and for use in system models. A summary, conclusions, and recommendations based on the present work are discussed in Chapter 6 . 


\section{Chapter 2}

\section{Literature Review}

\subsection{Introduction}

This project involved the development of a radial inflow turbine for an S-CO2 power cycle. This section will examine common S-CO2 power cycles to establish an understanding of the operating point of the turbine. To further the understanding of the power cycles, the real gas properties of the working fluid, S-CO2, will be briefly presented. This leads into a review of the purpose of the turbines in power cycles, and the selection between axial and radial turbines. This literature review will end with a review of radial turbine aerodynamic design, including design recommendations from literature.

\subsection{Supercritical $\mathrm{CO}_{2}$ Power Cycles}

The focus of this thesis is the design of the turbine for an S-CO2 cycle and the specifics of the corresponding cycle need not be addressed. However it is useful to know what S-CO2 cycles are and how they came to be. The history of S-CO2 power cycles up to the time of the writing of this thesis has been thoroughly reviewed by Crespi et al. (2017). Angelino $(1968,1969)$ and Feher $(1968 \mathrm{a}, \mathrm{b})$ are widely recognized for simultaneously being the first to recognize the advantages of supercritical power cycles. Both concluded that the supercritical cycle with carbon dioxide as the working fluid offered higher cycle thermal efficiencies at higher source temperatures $\left(>700^{\circ} \mathrm{C} / 973.15 \mathrm{~K}\right)$ than conventional cycles (e.g. steam Rankine 
cycles). Although the proposed cycles were theoretically promising, it was not until Dostal (2004) presented his research that the engineering community began to put more focus on this technology.

The supercritical phase can be defined as the state of a material when its pressure and temperature are both above the critical pressure and temperature. The phases of carbon dioxide with respect to its pressure and temperature are plotted in Figure 2.1. A supercritical cycle is one where the compression work is done above the critical point and the working fluid remains in the supercritical phase throughout the cycle. $\mathrm{CO}_{2}$ has a high density, almost half that of water, near the critical point allowing for lower compression work in this operating range. This has the result that the required power for compression is lower and the cycle efficiency may be higher.

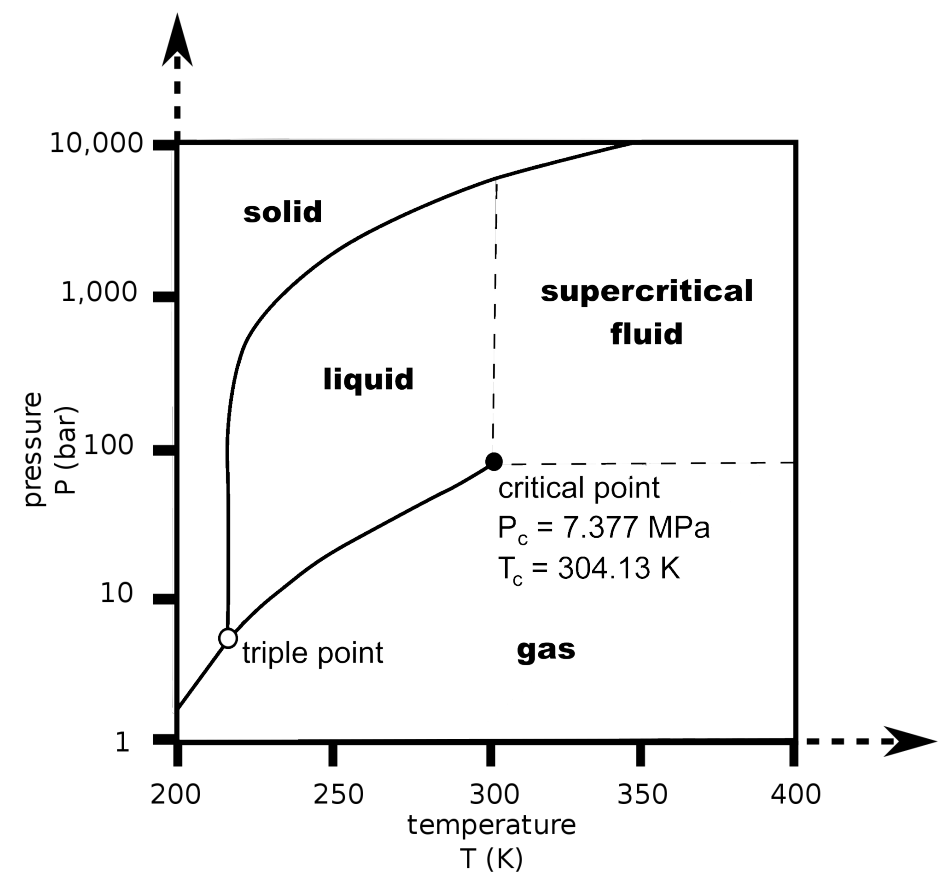

Figure 2.1: Phases of $\mathrm{CO}_{2}$ with respect to pressure and temperature (Adapted from Finney \& Jacobs, 2010). (Critical values obtained from Span \& Wagner (1996).)

The simplest form of an S-CO2 power cycle is the Brayton cycle. Feher (1968a) used a closed Brayton cycle to develop his original supercritical cycle. As Crespi et al. (2017) have shown, many cycle configurations have been based on Feher's supercritical cycle. For example, the simple regenerative Brayton cycle has been adopted by Carleton University for one of its 
fourth year undergraduate capstone projects. The Carleton University Brayton Cycle Loop (CUBCL) is shown schematically in Figure 2.2 and can be represented on an enthalpy-entropy diagram as shown in Figure 2.3 (both reproduced from Figure 1.2). The specifics of the cycle for the present project are not important because the focus is only the turbine aerodynamic design.

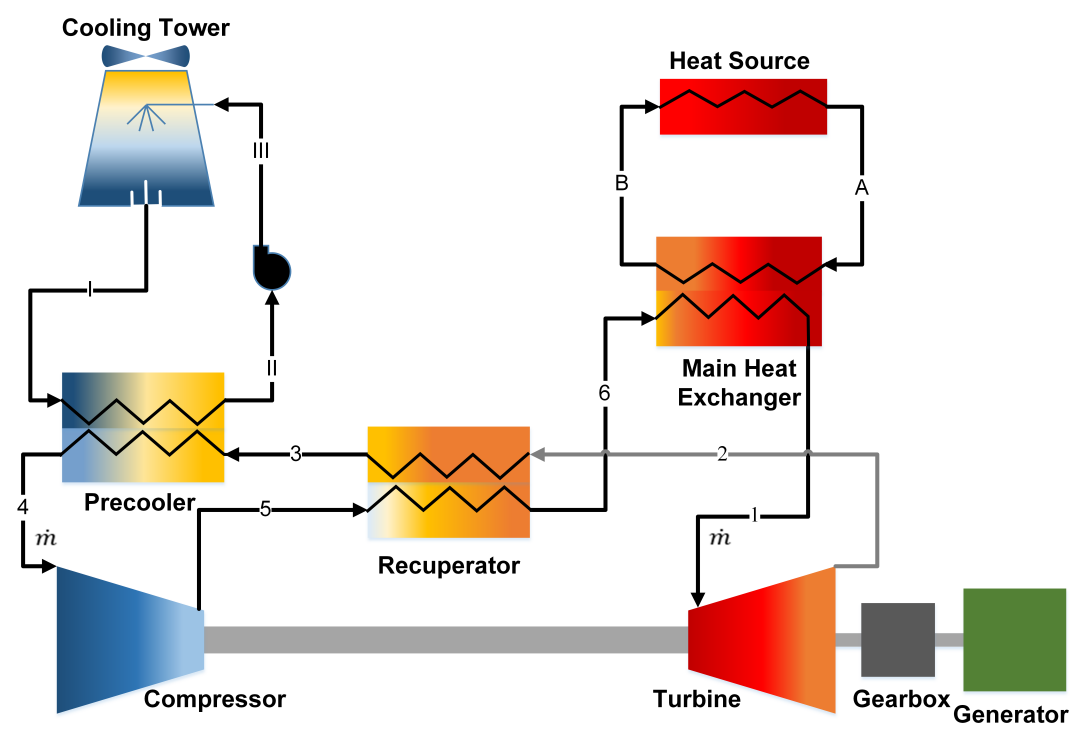

Figure 2.2: CUBCL simple regenerative Brayton cycle (Adapted from MacLean, 2012, by Alberto J. Martel Matos, Carleton University).

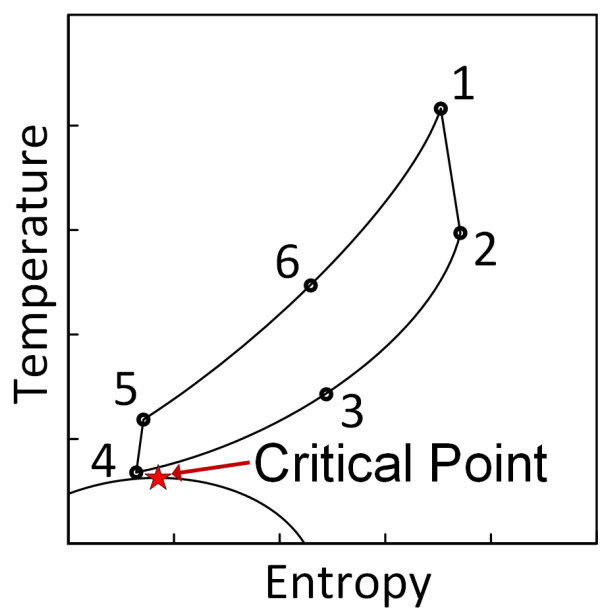

Figure 2.3: CUBCL simple regenerative Brayton cycle represented on a temperature versus entropy $(T-s)$ diagram (not to scale). 
The compressor, operating from state points 4 to 5 in this example cycle, operates close to the critical point (at the inlet) to reduce the required compressor work by taking advantage of the high density (Dostal, 2004). However, when a fluid is near the critical point, it does not act as an ideal gas and therefore this must be taken into account in the design of the compressor. The turbine for the S-CO2 plant operates in a region with higher compressibility, and the fluid behaves more like an ideal gas. Nevertheless, an understanding of the real gas behaviour of S-CO2 is still necessary when designing the turbine. For example, in Section 4.8, it is shown that CFD simulations using only ideal gas properties do not accurately capture the deviation of the flow after the trailing edge of the nozzle and rotor blades, so real gas properties should be used whenever possible when designing S-CO2 turbines.

\subsection{Fluid Properties for Supercritical Carbon Dioxide}

The physical properties of S-CO2 make it a good candidate for a power cycle, but presents challenges for designers and researchers. Figure 2.1 plots the phase diagram of carbon dioxide which has a critical point at 7.377 MPa and 304.13 K (Span \& Wagner, 1996). Near the critical point, the properties (in particular the density and specific heat capacity) change significantly with small changes in pressure or temperature.

Ideal gases are fluids that can be characterized by the ideal gas equation of state:

$$
P=\rho R T
$$

where $P$ is the static pressure, $\rho$ is the static density, $R$ is the specific gas constant, and $T$ is the static temperature. This relation, however, is not applicable for all pressure and temperature states of a gas and its error increases when a substance is near its critical point. The compressibility factor, $Z$, is used to define the deviation from ideal gas at a certain state of pressure and temperature. The compressibility factor is the ratio of the left-hand side to the right-hand side of the ideal gas equation of state.

$$
Z=\frac{P}{\rho R T}
$$


A compressibility factor of 1.0 indicates an ideal gas. The variation in the compressibility factor has been shown to correlate well with variation in the reduced temperature, $T_{R}$, and reduced pressure, $P_{R}$, where

$$
\begin{aligned}
T_{R} & =\frac{T}{T_{c}} \\
P_{R} & =\frac{P}{P_{c}}
\end{aligned}
$$

with $T_{c}$ being the critical temperature, and $P_{c}$ being the critical pressure. Figure 2.4 shows the variation of the compressibility factor with the reduced pressure and reduced temperature. Near the critical point, when $T_{R}$ and $P_{R}$ are near unity, the compressibility factor is less than unity and thus the gas is far from ideal.

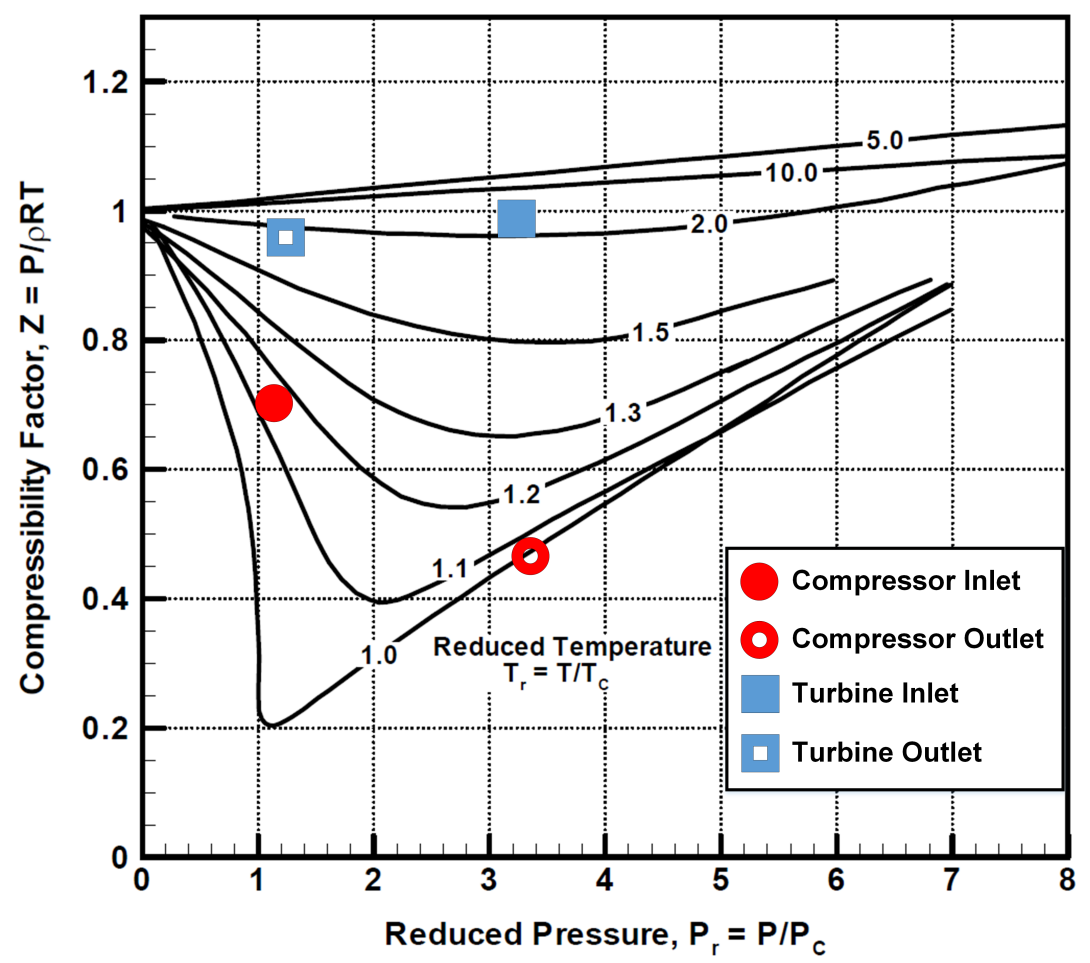

Figure 2.4: Compressibility factor versus reduced pressure and reduced temperature with example S-CO2 turbomachinery inlet and outlet points (Adapted from Sjolander, 2017).

The compressibility factor does not capture all real gas effects and therefore must not be used as a modifier to the ideal gas equation of state. It is simply an indication of the deviation 
from ideal gas behaviour and may be used by turbomachinery designers when applying literature recommendations, because most of them have been formulated with ideal gas working fluids. Example turbine and compressor inlet and outlet state points are plotted on Figure 2.4. For the present project, the compressibility factor at the inlet and outlet of the turbine were 0.995 and 0.960. In comparison, the compressibility factor for S-CO2 compressors can go as low as 0.5 or lower, meaning that the turbine operates in a region where the fluid acts more like an ideal gas.

\subsection{Modelling Supercritical Carbon Dioxide Properties}

Equations of state are used to determine the physical properties of a substance at a given state. For example, one might know the pressure and temperature of a fluid and want to know the density or specific heat capacity at that given state. Computational fluid dynamics (CFD) solvers, such as ANSYS CFX 18.1 which will be used to evaluate the radial turbine design, require equations of state to close the governing equations. The simplest equation of state is the ideal gas equation of state, which has already been presented as the following relationship:

$$
\rho=\frac{P}{R T}
$$

where here, the density, $\rho$, can be calculated for all states where the pressure, $P$, and temperature, $T$, are known.

To capture the real gas effects of fluids (as required by ANSYS CFX), more complex equations of state are required. White \& Weiland (2017) recognize the use of REFPROP as the best method for estimating $\mathrm{CO}_{2}$ fluid properties. REFPROP is the fluid reference property database created by the National Institute of Standards and Technology (NIST) which uses the most accurate equations of state currently available (Lemmon et al., 2013). For carbon dioxide, REFPROP uses the equation of state specified by Span \& Wagner (1996) which was particularly developed to capture the property variation around the critical point and in the supercritical region. The Span and Wagner equation of state includes complex equations with many coefficients, making it tedious to implement in codes and scripts for the design process. 
REFPROP allows designers to simply run its executable or scripts instead of writing and solving the full equations manually. This means that REFPROP can easily be implemented in the preliminary design process where a few calls to the fluid properties are required, but when more calls to the fluid properties are required, such as with CFD simulations, direct calls to REFPROP become computationally costly.

A popular method to reduce the computational cost associated with calls to REFPROP is the use of lookup tables, called RGP (real gas property) tables, which are generated prior to the CFD simulation with the desired refinement. Lookup tables in the form of .rgp files may be used directly with many CFD software, including ANSYS CFX and Fluent. Ameli et al. (2017), Saxena et al. (2017), and Zhang et al. (2017) showed how refined .rgp files may be used to successfully model the complex flow through S-CO2 compressors and turbines.

Finally, the physical properties of real gases may be estimated by cubic equations of state. ANSYS CFX has four real gas equations of state built in: the standard Redlich-Kwong (RK) method, the Aungier-Redlich-Kwong (ARK) method, the Soave-Redlich-Kwong (SRK) method, and the Peng-Robinson (PR) method. When choosing between the RK model (and models based on it) and the PR model to estimate $\mathrm{CO}_{2}$ properties, Mazzoccoli et al. (2012) showed that the PR model has less error when compared to experimental data. The mathematical model of this equation of state used by ANSYS CFX may be found in Appendix A. It was found that the accuracy of the cubic equation of state, was sensitive to the coefficients

used for the definition of $c_{\mathrm{p}_{0}}$, and large errors in entropy were observed as discussed in Appendix A. For this reason and with REFPROP available for the present work, the RGP table method was used for CFD simulations.

\subsection{Turbomachinery Machine Type Selection}

In power generation systems, the turbine is the component which extracts energy from the working fluid. Also, S-CO2 cycle calculations have shown that the overall cycle efficiency is more sensitive to turbine efficiency than the compressor efficiency (Feher, 1968b). This means that the turbine should receive adequate attention in the design phase to ensure that the 
maximum mechanical work is extracted. The two most common forms of turbines for power cycles are axial turbines and radial inflow turbines. The selection between these two types for a specific application is not trivial. Allison et al. (2017) cover the basis for selection of turbomachinery type for S-CO2 plants and discuss the selected machine types for various prototype plants and plants from literature. When selecting which type of turbomachinery to use for their application, designers can use non-dimensional parameters to utilize empirical data relating to previously successful designs. Non-dimensional parameters allow the comparison of geometrically and dynamically similar flows. Specific speed is a non-dimensional parameter which is commonly used in the preliminary stage to select a turbine type. Specific speed is represented as

$$
\Omega=\frac{\omega Q^{\frac{1}{2}}}{(g \Delta H)^{\frac{3}{4}}}
$$

where $\omega$ is the angular velocity in $\mathrm{rad} / \mathrm{s}, Q$ is the volumetric flow rate, $g$ is the acceleration due to gravity, and $\Delta H$ is the head change. This specific speed may be used directly to make a preliminary selection between an axial or radial turbine by using the chart shown in Figure 2.5.

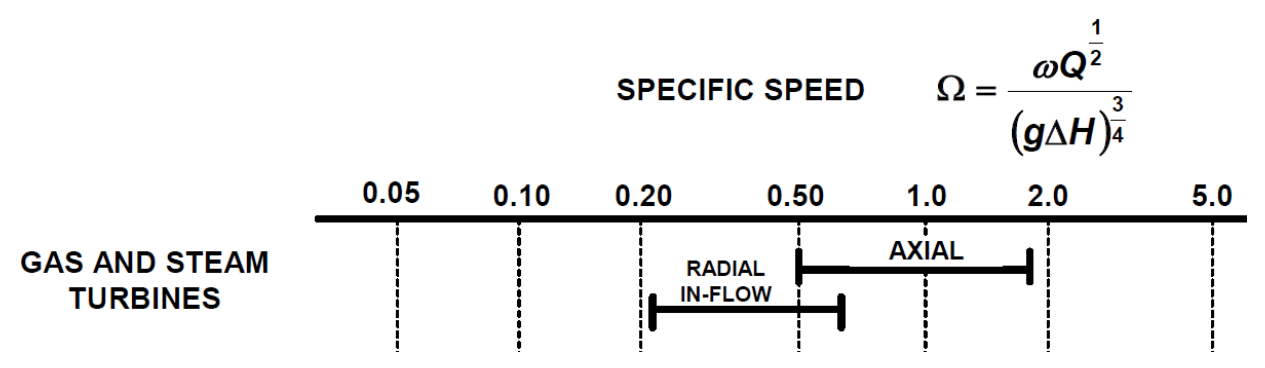

Figure 2.5: Specific speed chart for preliminary turbomachine type selection (Adapted from Csanady (1964)by Sjolander (2017)).

The information regarding machine type and corresponding plant output power presented by Allison et al. (2017) is shown in Table 2.1. Allison et al. (2017) discussed how radial turbines are likely used for plants with output powers below $10 \mathrm{MW}_{\mathrm{e}}$, and axial turbines are favoured for higher powered plants. With a plant output power of $10 \mathrm{MW}_{\mathrm{e}}$, the clients of this project chose radial inflow as the turbine machine type. From here on "radial inflow turbines" may simply be referred to as "radial turbines". 
Table 2.1: S-CO2 turbine machine type corresponding to various proposed and existing plants with a range of output powers (Data obtained from Allison et al., 2017).

\begin{tabular}{|c|c|c|c|c|}
\hline & $\begin{array}{l}\text { Original } \\
\text { Source }\end{array}$ & $\begin{array}{r}\text { Plant Output } \\
\text { Power }\end{array}$ & $\begin{array}{r}\text { Turbine } \\
\text { Machine Type }\end{array}$ & $\begin{array}{l}\text { Number } \\
\text { of Stages }\end{array}$ \\
\hline \multirow{4}{*}{$\begin{array}{l}\text { Current } \\
\text { Prototypes }\end{array}$} & Wright et al. (2010) & $125 \mathrm{~kW}_{\mathrm{e}}$ & $2 \mathrm{RT}$ & 1 \\
\hline & $\begin{array}{l}\text { Kimball \& } \\
\text { Clementoni (2012) }\end{array}$ & $100 \mathrm{~kW}_{\mathrm{e}}$ & $2 \mathrm{RT}$ & 1 \\
\hline & Held (2015) & $8 \mathrm{MW}_{\mathrm{e}}$ & $2 \mathrm{RT}$ & 1 \\
\hline & Moore et al. (2015) & $10 \mathrm{MW}_{\mathrm{e}}$ & $1 \mathrm{AT}^{*}$ & 4 \\
\hline \multirow{10}{*}{$\begin{array}{l}\text { Theoretical or } \\
\text { Future Projects }\end{array}$} & Wilkes et al. (2016) & $5-25 \mathrm{MW}_{\mathrm{e}}$ & $1 \mathrm{RT}$ & 4 \\
\hline & Iwai et al. (2015) & $25 \mathrm{MW}_{\mathrm{e}}$ & $1 \mathrm{AT}$ & 7 \\
\hline & \multirow[t]{2}{*}{ Bidkar et al. (2016a,b) } & $50 \mathrm{MW}_{\mathrm{e}}$ & $1 \mathrm{AT}$ & 6 \\
\hline & & $450 \mathrm{MW}_{\mathrm{e}}$ & 1DAT & 7 \\
\hline & Dostal (2004) & $246 \mathrm{MW}_{\mathrm{e}}$ & $1 \mathrm{AT}$ & 3 \\
\hline & Angelino (1968) & $1000 \mathrm{MW}_{\mathrm{e}}$ & 1DAT & 9 \\
\hline & \multirow[t]{2}{*}{ McDowell et al. (2015) } & $10 \mathrm{MW}_{\mathrm{e}}$ & 1DAT & 4 \\
\hline & & $550 \mathrm{MW}_{\mathrm{e}}$ & 1DAT & 4 \\
\hline & Thimsen (2013) & $645 \mathrm{MW}_{\mathrm{e}}$ & 1DAT & 4 \\
\hline & Johnson et al. (2012) & $1000 \mathrm{MW}_{\mathrm{e}}$ & 1DAT & 4 \\
\hline
\end{tabular}

Note: $2 \mathrm{RT} \equiv$ two radial turbines ( 1 compressor drive turbine, 1 power turbine), $1 \mathrm{RT} \equiv$ one radial turbine, $1 \mathrm{AT} \equiv$ one axial turbine, $1 \mathrm{DAT} \equiv$ one double-flow axial turbine.

* axial type selected to allow for scaling up to $50 \mathrm{MW}_{\mathrm{e}}$ 


\subsection{Radial Inflow Turbine Aerodynamic Design}

The design of turbomachinery begins with a preliminary design where the initial size of each component is determined. This initial sizing is based on the required performance considering the working fluid properties and any other constraints, such as rotational speed.

Research in the 1960's by researchers at NASA such as Wood (1963), Kofskey \& Wasserbauer (1966), Nusbaum \& Kofskey (1969), and Rohlik (1968) is widely credited for the methods used for radial turbine design, even today. Aungier (2006), Japikse \& Baines (1994), Whitfield \& Baines (1990), and others have compiled design recommendations into a form which may be used to develop a complete preliminary design for a radial turbine. White (2015) provided a thorough outline of the details of radial turbine design for real gases, from the preliminary sizing to the detailed CFD analysis and off-design modelling. This section will outline the preliminary design methods in literature and detailed design methods such as the use of CFD.

\subsubsection{Background}

Turbines comprise a stator, a stationary component that imparts swirl on the fluid, and a rotor, a rotating component which extracts energy from the fluid by removing the swirl. A radial turbine is illustrated in Figure 2.6 including a stator composed of a scroll volute and a nozzle passage, or simply a vaned volute. Scroll volutes are used when the flow approaching the turbine is in a pipe. The flow enters the volute tangential to the circumference of the rotor at station 1 and is then turned through the scroll to be distributed uniformly around the circumference at station 2. If the incoming flow is in an annular passage (for example, if the turbine follows a combustor), then a volute is not required. In the illustrated turbine, after leaving the volute, the flow moves radially towards the axis of rotation through the nozzle passage from station 2 to 3 . In some cases, the volute can deliver a circumferentially uniform flow with enough swirl to the rotor inlet meaning that nozzle vanes are not required. However, volutes are usually only capable of delivering the required flow angle at the design point operating conditions. In off-design cases, vanes are required to impart the required flow angle in the stationary frame. Japikse \& Baines (1994) includes a more detailed discussion on 
the selection of stators for radial turbines and the need for vanes. This project used a vaned volute because power cycles require adequate performance over a wide range of operating conditions (through startup and shutdown procedures for example) and the incoming flow to the turbine will be through a pipe.
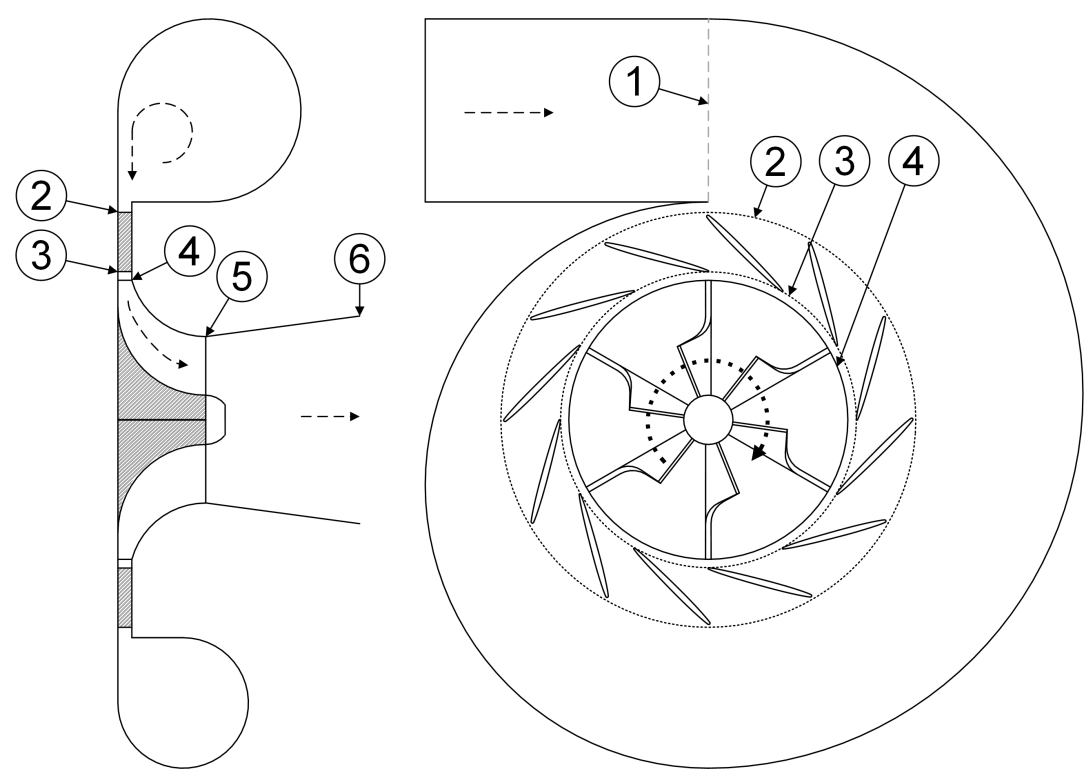

Figure 2.6: Radial turbine components and station locations for sizing.

Following the vaned volute stator is the rotor from station 4 to 5 . The rotor takes the radially flowing fluid and turns it through $90^{\circ}$. The rotor is where the energy is extracted from the fluid, so it receives the most design effort. Neglecting frictional effects, the rotor is the only turbine component which does work on the fluid. The rotor work is negative because it extracts energy from the fluid. The rotor exhaust optionally passes through a diffuser to recover static pressure from the rotor exit velocity; however, a diffuser was not investigated for this project because the overall plant layout will be a key limiting factor for the diffuser sizing in terms of length and it was assumed that the other components would have a larger effect on the performance.

Figure 2.7 shows the flow through the turbine when represented thermodynamically on an enthalpy-entropy $(h-s)$ diagram with both the total (stagnation) and static conditions. The difference between total and static conditions is best described in terms of enthalpy. This 


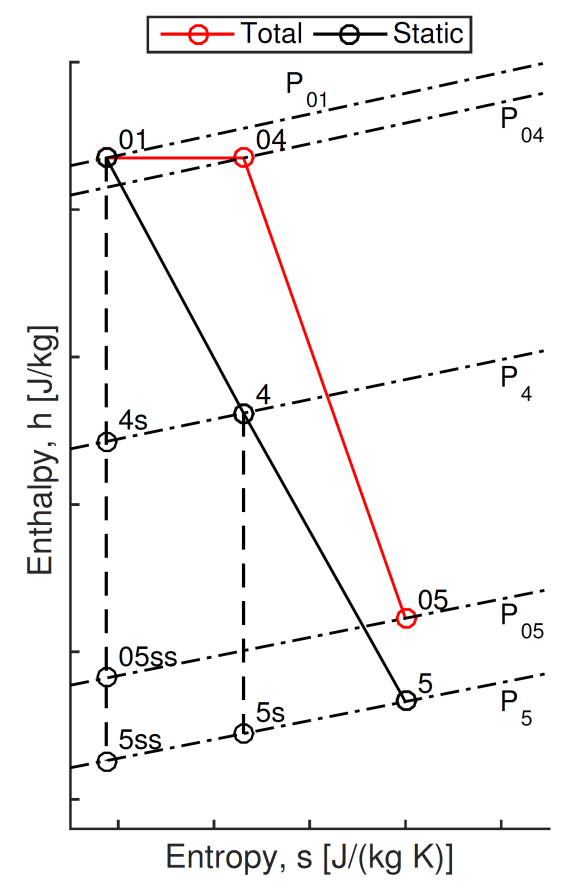

Figure 2.7: General $h$-s diagram for the expansion process through a turbine with state points corresponding to Figure 2.6 (White, 2015).

leads to the definition of total enthalpy in Equation 2.7.

$$
h_{0}=h+\frac{1}{2} C^{2}
$$

where $h_{0}$ is the total enthalpy, $h$ is the static enthalpy, and $C$ is the absolute velocity of the fluid.

\section{Euler Turbomachinery Equation}

The fundamental equation for the performance of turbomachinery is the Euler equation. First, the steady flow energy equation for turbomachines (assuming adiabatic flow, neglecting potential energy effects, and assuming uniform 1-D flow) is shown to be (Dixon \& Hall, 2013):

$$
\dot{W}_{\text {shaft }}=\dot{m}\left(h_{02}-h_{01}\right)
$$


where $\dot{W}_{\text {shaft }}$ is the work done by the turbomachine on the fluid, $\dot{m}$ is the mass flow rate, and $h_{01}$ and $h_{02}$ are the mass-averaged total enthalpy values at the inlet and outlet of the machine (station 1 and 5 in Figure 2.7). Turbines act to remove energy from the working fluid, so the work term by this definition would be negative (i.e. $h_{01}>h_{02}$ therefore $\dot{W}_{\text {shaft }}<0$ ), however, it is common to simply define the turbine work, $\dot{W}_{t}$, as the negative of the shaft work.

$$
\dot{W}_{t}=-\dot{W}_{\text {shaft }}=\dot{m}\left(h_{01}-h_{02}\right)
$$

In addition, the angular momentum equation is derived with the understanding that the energy transferred from the fluid to the rotor causes a change in the fluid's angular momentum and results in a torque on the rotor (Wright \& Gerhart, 2009). Assuming uniform 1-D flow again, this principle is expressed mathematically as :

$$
T=\dot{m}\left(\left(r C_{\theta}\right)_{\text {in }}-\left(r C_{\theta}\right)_{\text {out }}\right)
$$

where $T$ is taken as the torque applied to the rotor by the fluid, $r$ is the mean radius, and $C_{\theta}$ is the tangential velocity. When this torque is multiplied by the angular velocity of the shaft, $\omega$, the shaft power is obtained, $\dot{W}=T \omega$. Also, knowing the blade speed, $U=r \omega$, the Euler turbomachinery equation is derived from Equations 2.9 and 2.10 as follows:

$$
h_{01}-h_{02}=U_{1} C_{\theta 1}-U_{2} C_{\theta 2}
$$

This equation relates the required enthalpy change to the required blade speed and tangential velocity changes through the turbine. This is the total enthalpy change seen through the turbine including all losses, therefore it differs from the enthalpy drop required from the design specifications for power output and mass flow rate.

\section{Turbine Isentropic Efficiency}

The isentropic efficiency of a turbine is simply the ratio of the actual work done on the turbine to the work done on the turbine for the same total pressure change if the process 
were isentropic (with no losses). The isentropic process through a turbine is represented in Figure 2.7 by the dashed line from point 01 through $05 s s$ and $5 s s$. On this same figure, the actual process is represented by the solid lines from point 01 through point 05 or point 5 . As indicated by the figure, the state points preceded by 0 indicate total conditions, otherwise they are static conditions. The difference between total and static enthalpy is represented in Equation 2.7.

When evaluating the isentropic efficiency, it is important to distinguish between the comparison of total-total conditions or total-static conditions. The flow at the exit of the turbine still contains kinetic energy and the difference between total-total and total-static isentropic efficiencies is whether the kinetic energy is recovered or not (White, 2015). The total-total efficiency assumes that the kinetic energy is recoverable and directly compares the actual enthalpy change to the ideal enthalpy change for total-total conditions.

$$
\eta_{t t}=\frac{h_{01}-h_{05}}{h_{01}-h_{05 s s}}
$$

The total-static isentropic efficiency is defined with the argument that the kinetic energy remaining in the fluid at the turbine exit is not recoverable. Therefore, the ideal work is defined using total-static conditions.

$$
\eta_{t s}=\frac{h_{01}-h_{05}}{h_{01}-h_{5 s s}}
$$

\section{Rotor Geometry and Velocity Diagrams}

The rotor is where the work is performed, so it is where the design process begins. The rotor of a radial turbine takes the form illustrated in Figure 2.8. The main dimensions obtained from the preliminary design process for the rotor are shown.

In Figure 2.8, the dashed line through rotor flow passage represents the meanline. At the preliminary design stage, turbomachinery are generally analyzed using meanline analysis. Meanline analysis assumes one-dimensional flow by only considering the flow at the mean line which divides the cross-section in half. This assumption ignores secondary flows, boundary 


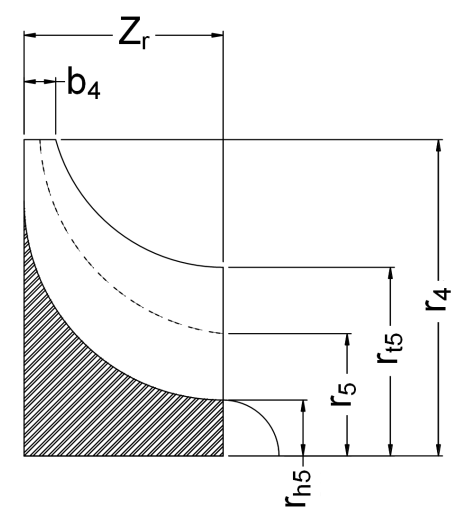

Figure 2.8: Radial turbine rotor sizing dimensions.

layer effects, and radial differences, but these limitations are accepted at the preliminary design stage. In meanline analysis, the rotor outlet is designed by taking the mean radius as the root mean square radius by convention (Japikse \& Baines, 1994). The root mean square radius for the rotor exit is calculated as follows:

$$
r_{5}=\sqrt{\frac{r_{h 5^{2}+r_{t 5}^{2}}}{2}}
$$

where the $r_{h 5}$ is the radius at the outlet hub and $r_{t 5}$ is the radius at the tip.

As shown by the Euler equation, the tangential component of the mass-averaged velocity at the inlet and outlet of the rotor determine the performance of the turbine. Velocity diagrams are vector plots of the meanline velocity at specified points, such as the inlet and outlet of the rotor. These velocity diagrams include the absolute velocity vector in the stationary frame, $C$, the relative velocity vector relative to the rotating frame, $W$, and the blade speed, $U$, to connect the stationary and rotating frame. Figure 2.9 depicts the inlet velocity diagram where the flow enters the rotor with an absolute velocity, $C_{4}$, in the stationary frame at an angle of $\alpha_{4}$ with respect to the radial direction towards the axis. This absolute velocity is composed of the meridional component, $C_{m 4}$, which is assumed to be completely radial, and the tangential component, $C_{\theta 4}$. The difference between $C_{\theta 4}$ and the blade speed, $U_{4}$, define the tangential component of the velocity in the relative frame, which along with the meridional velocity, define the velocity in the relative frame, $W_{4}$. This relative velocity approaches the blade at 
an angle of $\beta_{4}$. The rotor outlet velocity triangle is similar to the inlet triangle. The flow leaves the rotor with a velocity, $C_{5}$, at an angle of $\alpha_{5}$ with respect to the axial direction. This rotor outlet absolute velocity angle, $\alpha_{5}$, is normally designed to be zero at the design point to minimize losses due to swirl in the diffuser downstream of the rotor. The outlet meanline blade speed, $U_{5}$, connects the relative velocity, $W_{5}$, to the absolute velocity. The flow in the relative frame leaves the rotor at an angle of $\beta_{5}$.

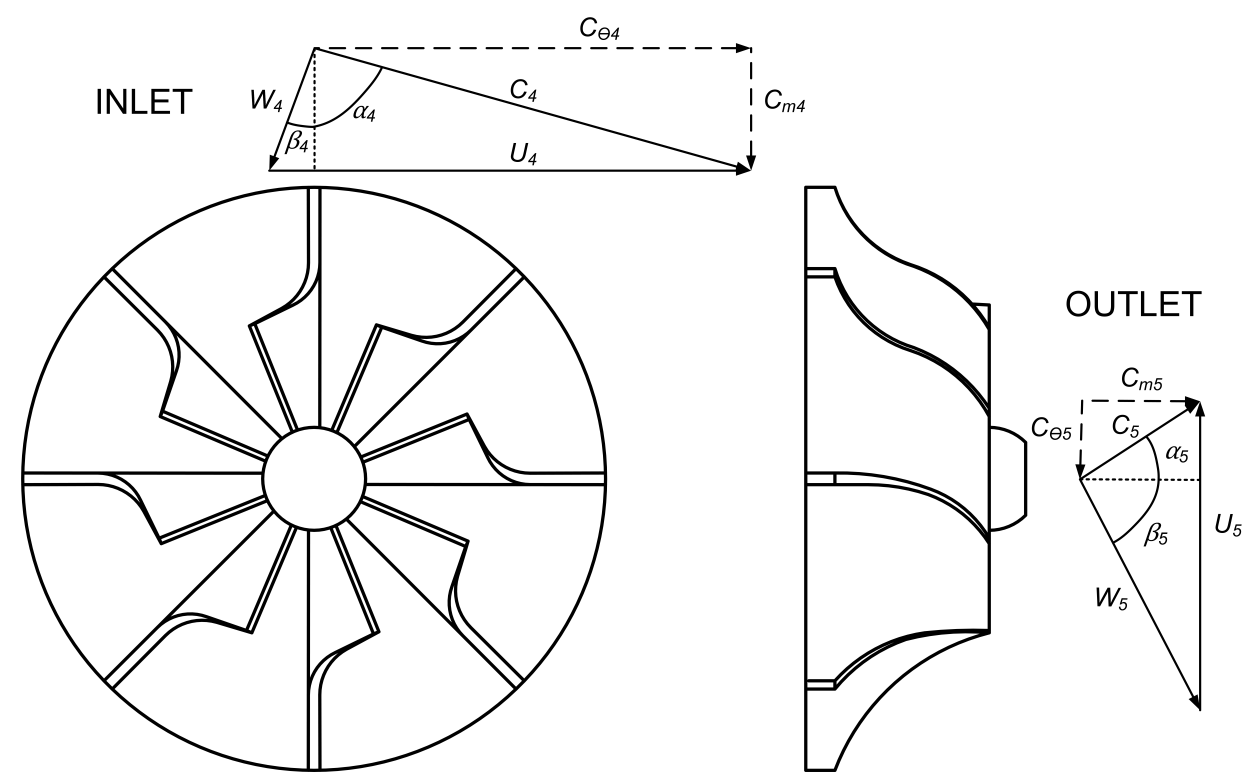

Figure 2.9: Radial turbine rotor example velocity diagrams.

In the preliminary stage, designers can use historical empirical data as reference for their design. The Work and Flow Coefficients are non-dimensional parameters which allow for the comparison of similar flows. The Flow Coefficient is defined as the ratio of the outlet meridional velocity, $C_{m 5}$ to the inlet blade speed, $U_{4}$, as in Equation 2.15.

$$
\Phi=\frac{C_{m 5}}{U_{4}}
$$

The Work Coefficient, also called the blade loading coefficient, is defined as the ratio of total enthalpy drop in the rotor, $\Delta h_{0}$, to the squared blade speed at the rotor inlet, $U_{4}=r_{4} \omega$. Japikse \& Baines (1994) also used the Euler equation to define the Work Coefficient instead 
in terms of the tangential velocities.

$$
\Psi=\frac{\Delta h_{0}}{U_{4}^{2}}=\frac{C_{\theta 4}}{U_{4}}-\frac{1}{\epsilon} \frac{C_{\theta 5}}{U_{4}}
$$

where $\epsilon=r_{4} / r_{5}$ is called the radius ratio. Radial turbine rotors are commonly designed with minimal exit swirl, so $C_{\theta 5}$ is small compared to $C_{\theta 4}$, allowing the Work Coefficient to be approximated as:

$$
\Psi \approx \frac{C_{\theta 4}}{U_{4}}
$$

Japikse \& Baines (1994) present a plot of Work (loading) Coefficient versus Flow Coefficient with lines of constant total-static isentropic efficiency based on empirical data as shown in Figure 2.10. This chart indicates that maximum efficiency occurs with Work Coefficients between $0.8-1.0$ and Flow Coefficients between $0.2-0.3$ (Japikse \& Baines, 1994).

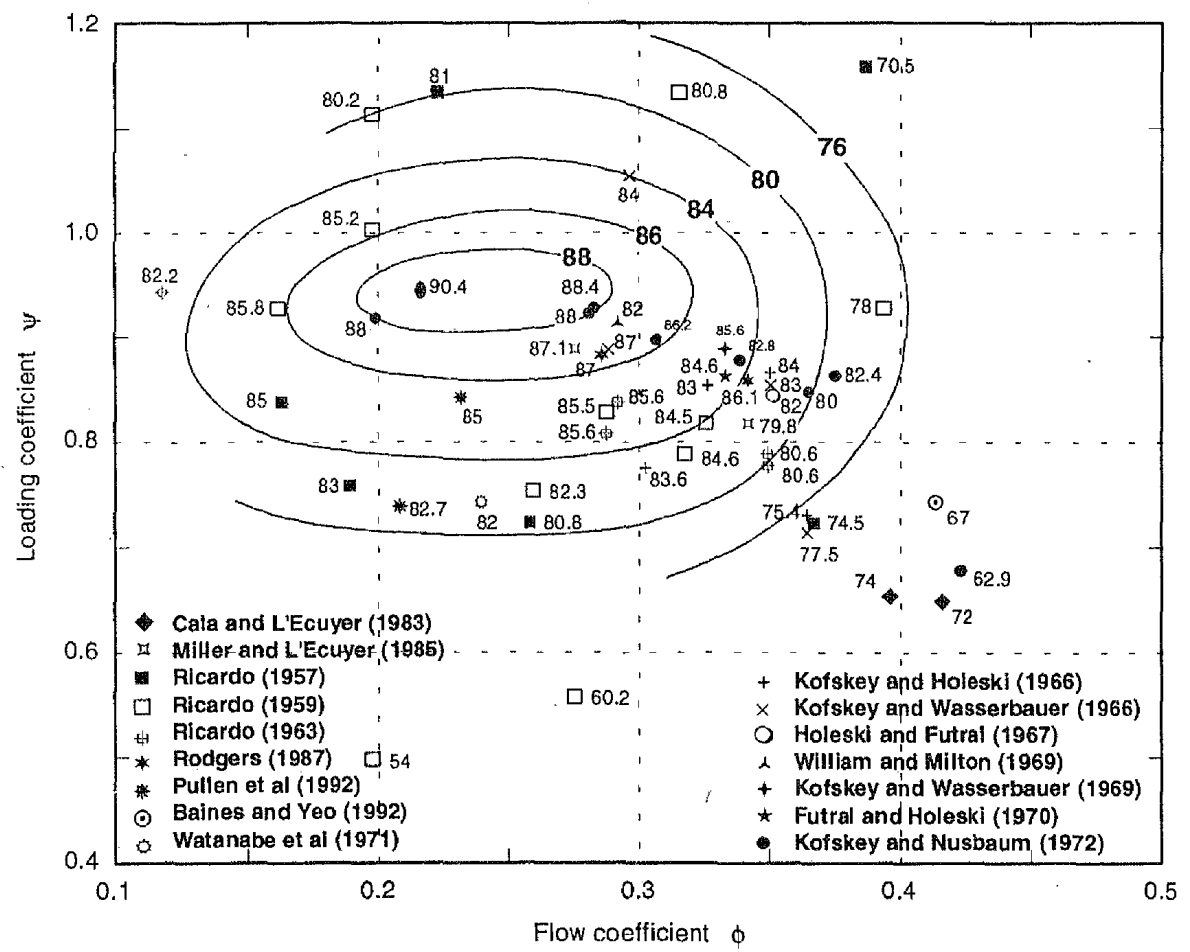

Figure 2.10: Work Coefficient versus Flow Coefficient with lines of constant total-static efficiency (Japikse \& Baines, 1994). 
High values of Work Coefficient are associated with highly loaded blades which may be more prone to stall, but low values indicate that the rotor is not taking full advantage of the blade speed. The ideal value of Work Coefficient is estimated by assuming an ideal efficiency, $\eta_{t s}=1$, and the optimum isentropic velocity ratio, $\nu_{t s}$, in the following form of Work Coefficient from (Japikse \& Baines, 1994):

$$
\Psi=\frac{\eta_{t s}}{2 \nu_{t s}^{2}}
$$

The isentropic velocity ratio is simply $\nu_{t s}=U_{4} / C_{s}$, where $C_{s}$ is the spouting velocity. The spouting velocity is defined as the theoretical velocity required for a kinetic energy equal to the enthalpy drop through the turbine if the process were isentropic (White, 2015), represented as:

$$
\frac{1}{2} C_{s}^{2}=h_{01}-h_{5 s s}=\Delta h_{0 s s}
$$

Under ideal conditions, it can be shown that the isentropic velocity ratio $\nu_{t s}=0.707$, but Dixon \& Hall (2013) referenced that real turbines operate around $0.68<\nu_{t s}<0.71$. This leads to an optimum Work Coefficient of 1.

\section{Nozzle Geometry}

After the preliminary rotor design, the nozzles are designed based on the rotor inlet design. As part of the stator component of the turbine, the nozzles act to accelerate the flow and impart some swirl. The preliminary nozzle design includes the specification of the radial distance from the nozzle outlet to the rotor inlet, the shape of the nozzle blades, and the nozzle inlet and outlet radii. Figure 2.11 indicates the main dimensions associated with an uncambered nozzle row design. In the preliminary stage, Aungier (2006) suggested that a simple uncambered blade is favoured and therefore this was used for this project. Also, Aungier (2006) recommended maintaining a uniform width of the radial passage from the volute outlet, $b_{2}$, to the rotor inlet, $b_{4}$. The velocity increases through the nozzle passage mainly due to the reduction in circumferential area from station 2 to station 4 . At the design point the volute imparts most of the required swirl, but the nozzle passage is required to control swirl at off-design conditions (Japikse \& Baines, 1994). 

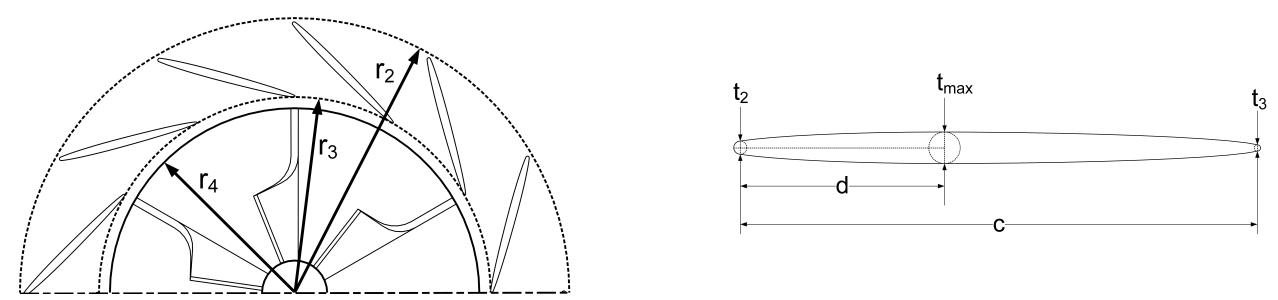

Figure 2.11: General radial turbine nozzle dimensions.

\section{Volute Geometry}

The volute preliminary design follows the nozzle design, as the sizing and velocity information defines the sizing of the volute outlet. This project includes a scroll volute where the incoming flow approaches the turbine tangential to the rotor circumference. The purpose of the volute is to distribute the flow uniformly around the nozzle passages, and impart swirl and accelerate the flow in the process. The volute geometry in this project followed the recommendations from Aungier (2006), featuring the general radial dimensions and cross section shown in Figure 2.12. Station 2 represents the nozzle inlet radius and station $2 \mathrm{v}$ represents the volute outlet radius which is radially offset from station 2 by a vaneless passage to allow for better mixing out.

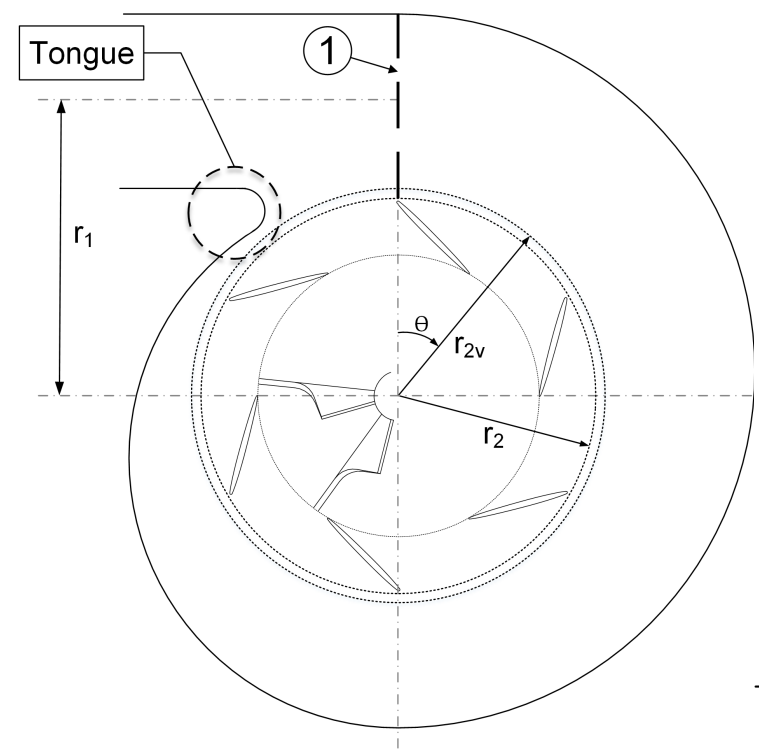

(a) Volute radial locations.

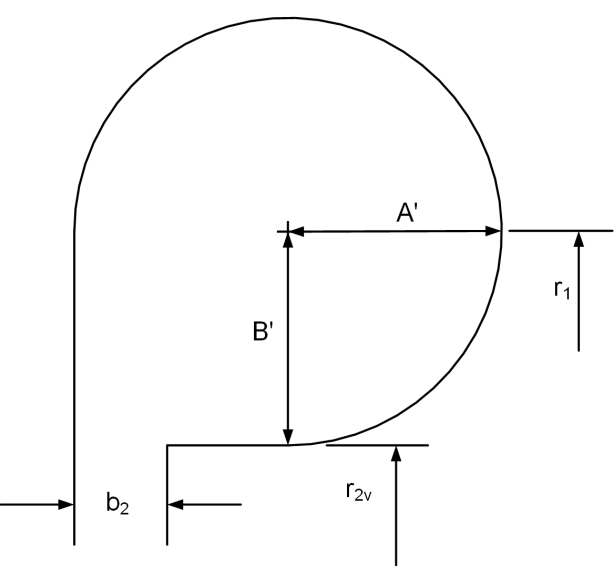

(b) Volute cross section.

Figure 2.12: General radial turbine scroll volute dimensions. 
A feature of scroll volutes is the tongue, which is the point where flow which has completed a full revolution around the circumference, recirculates with the incoming inlet flow. Qiang et al. (2010) recommended tongue locations of $-40^{\circ}$ to $-50^{\circ}$ azimuth from the station 1 plane.

\section{Stator Performance}

The performance of the stator, volute and nozzles, can be compared to those in literature to determine whether the design is successful or not. The volute and nozzle pressure loss coefficient, Equation 2.20 and Equation 2.21, quantify the losses through the component with lower values indicating less losses. Japikse \& Baines (1994) stated that successful radial turbines have volutes with $K_{V}$ values between 0.1 and 0.3 , and nozzles with $K_{N}$ values between 0.03 and 0.07 .

$$
\begin{aligned}
K_{V} & =\frac{P_{01}-P_{02}}{P_{02}-P_{2}} \\
K_{N} & =\frac{P_{02}-P_{03}}{P_{03}-P_{3}}
\end{aligned}
$$

\subsubsection{CFD Analysis}

Computational fluid dynamics (CFD) is a useful tool to further the design of turbomachinery. The flow through the turbine passage can be analyzed to identify areas where losses are generated and where design changes may be required to increase overall efficiency. ANSYS CFX 18.1 is a common CFD solver that features turbomachinery-specific tools and has been used for this project.

The ANSYS CFX-Solver Theory Guide by ANSYS, Inc. (2017b) covers the governing equations which are solved and details the theory behind CFD solutions. ANSYS CFX 18.1 solves the Reynolds-averaged Navier-Stokes equations instead of the full Navier-Stokes equations to reduce computational cost by averaging turbulent flow values. 


\section{Turbulence Modelling}

The Direct Numerical Simulation of turbulence flows using the full Navier-Stokes equations is computationally costly, so turbulence models are implemented to reduce the computational cost (ANSYS, Inc., 2017b). CFD utilizes Reynolds Averaged Navier-Stokes equations to account for the turbulent flows with less computational cost by adding so-called Reynolds stresses. A common method of modelling turbulence in CFD simulations is an Eddy Viscosity model, where the Reynolds stresses in continuously forming and dissipating eddies are proportional to the velocity gradients by the gradient hypothesis (ANSYS, Inc., 2017b).

This Eddy Viscosity model is implemented in various turbulence models. The simplest models are Zero Equation models which provide fast convergence but are limited in accuracy. Two-equation turbulence models are the most commonly used. ANSYS CFX 18.1 includes the $k-\epsilon, k-\omega$, and $k-\omega$ SST two-equation turbulence models. The $k-\epsilon$ model is said to be the most robust and least computationally costly, but this is due to the fact that it uses Scalable Wall Functions to model the boundary layer (ANSYS, Inc., 2017b). Wall Functions assist these models with resolving the effect of shear stress on the boundary layer of walls by approximating the buffer layer and viscous sublayer and thus reducing the computational requirement (ANSYS, Inc., 2017b). The $k-\omega$ model is said to model the near wall flows better than the $k-\epsilon$ model because it uses Automatic Wall Functions which switch between scalable functions and low Reynolds number wall functions (ANSYS, Inc., 2017b), where the low Reynolds number refers to the flow in the boundary layer and not necessarily the flow through the machine. Generally, the $k-\epsilon$ model is said to better model freestream flows, while the $k-\omega$ model is said to better model near wall flows, and the $k-\omega$ SST model combines the $k-\epsilon$ freestream treatment with the $k-\omega$ near wall treatment.

ANSYS, Inc. (2017a) and Galindo et al. (2013) recommend the $k-\omega$ SST model for the most accurate turbomachinery simulations. However, the $k-\omega$ SST model requires a $y^{+}$ around 1 to fully resolve the boundary layer. $y^{+}$is a measure of the spacing of the first node from a wall in relation to the flow's wall shear stress and density, defined in Equation 2.22. A $y^{+}$around 1 may not be achievable in some situations because it can require a very fine mesh 
and lead to a computationally challenging simulation. Simpson et al. (2013) suggested that the $k-\omega$ SST model with Automatic Wall Functions could be used with $y^{+}$values up to 30 . Scalable Wall Functions are more applicable than Automatic Wall Functions when $y^{+}>30$ because the low Reynolds number wall functions are not applicable. In this case, if $y^{+}>30$, the $k-\epsilon$ model is most applicable because it is less computationally costly than the $k-\omega \mathrm{SST}$ model and is more robust than the $k-\omega$ model. For example, White (2015) examined the real gas flow through an ORC radial turbine and found that the $k-\omega$ model had negligible effect on the simulated flow compared to the $k-\epsilon$ model, so they used the $k-\epsilon$ model for robustness in their simulations. Sauret \& Gu (2014) discussed the upper limit for the $y^{+}$ value for the $k-\epsilon$ model, which is said to be related to the machine Reynolds number, $R e_{M}$ (Equation 2.23). For example, Sauret \& Gu (2014) used $y^{+}=703$ for their radial turbine with a $R e_{M}=8.8 \times 10^{7}$.

$$
y^{+}=\sqrt{\frac{\tau_{w a l l}}{\rho}}\left(\frac{\Delta y}{\nu}\right)
$$

where $\tau_{\text {wall }}$ is the wall shear stress, $\Delta y$ is the distance from the wall to the nearest computational node, and $\nu$ is the kinematic viscosity.

$$
\operatorname{Re}_{M}=\frac{\rho_{4} U_{4} r_{4}}{\mu_{4}}
$$

where $\rho_{4}$ is the rotor inlet density and $\mu_{4}$ is the rotor inlet dynamic viscosity.

\subsubsection{Radial Turbine Performance Characteristics}

As part of a real power cycle, the turbine will not always operate at the design conditions. The off-design conditions of the turbine may include variations in the rotational speed, mass flow rate, and inlet conditions (pressure and temperature). For this reason, it is normal to estimate how the designed turbine will perform at these off-design conditions. System models can use performance characteristics, which plot non-dimensional parameters, to estimate the effect of operating at off-design conditions on the turbine's performance (in terms of pressure ratio or efficiency, for example). Dixon \& Hall (2013) cover performance characteristics in more detail along with the application of similitude theory. Common turbine performance maps 
include the variation of pressure ratio, efficiency, or enthalpy rise ratio with non-dimensional mass-flow and rotational speed. Chapter 5 will discuss the non-dimensional parameters used for this project in more detail. As an example, a turbine map is shown in Figure 2.13 with the turbine's pressure ratio plotted against the corrected mass flow at various corrected speeds.

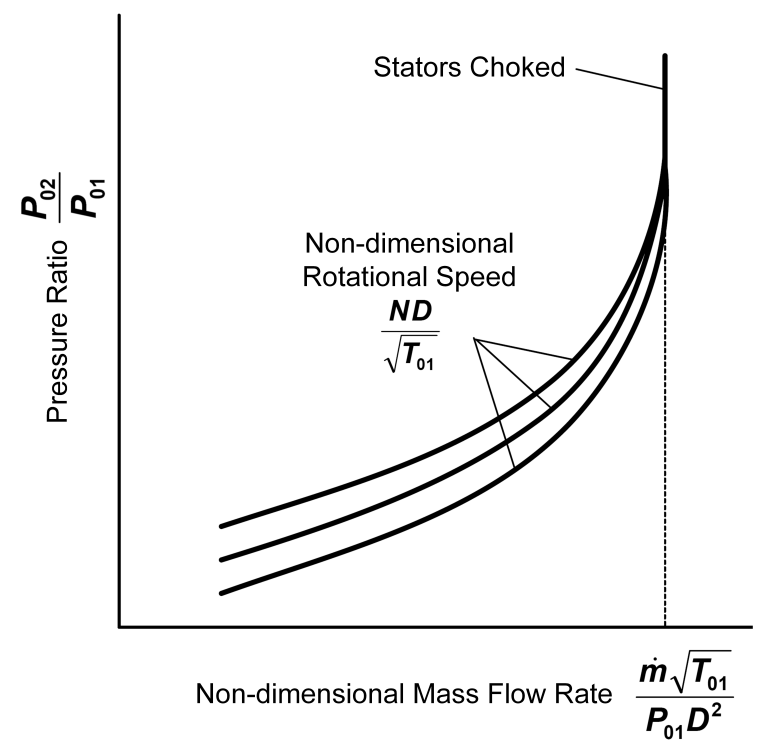

Figure 2.13: An example turbine map plotting the turbine's performance in terms of pressure ratio versus the mass flow parameter (Adapted from Sjolander, 2017).

It is desirable to develop turbine performance characteristics or turbine maps from experimental data, as the data will reflect what is actually built and will include all components, but this is hardly feasible at the design stage. Also, there are no quality published experimental data for S-CO2 cycles due to the resurgence in interest being only relatively recent, so other methods are needed to develop these turbine maps. CFD can be used by simply varying the boundary conditions of the simulation for a given geometry and working fluid. 


\section{Chapter 3}

\section{Radial Turbine One-Dimensional Development}

\subsection{Introduction}

A power cycle's turbine is where the power is generated. Turbines include stationary components, stators, which act to accelerate the working fluid and impart swirl, but do not extract energy from the fluid. Following the stators, the working fluid does work on the rotor, applying a torque as defined by Equation 2.10. The work extracted by the rotor is equal to the reduction in angular momentum in the working fluid, as shown by the Euler equation (Equation 2.11). A radial inflow turbine was selected for the overall project that the present work was performed for.

The components of radial inflow turbines include an inlet scroll volute, a nozzle passage, a rotor, and an outlet diffuser. This section will outline one-dimensional design methods for the volute, nozzles, and rotor. In the present work, the one-dimensional design is based on whole turbine efficiency values based on estimates of the losses in the individual components completed previously by a colleague. The stators, the volute and nozzles, are assumed to be isentropic, such that all the losses in the turbine come from the rotor. Recommendations from literature are used to guide this preliminary design and the real gas properties of the fluid are accounted for in the present procedure. The result of this chapter is a preliminary design of the radial turbine components in terms of the basic sizing and the inlet and outlet flows. 


\subsection{One-Dimensional Design Methodology}

The methodology used for the turbine preliminary design will be described in this section. The specific turbine designed for this project will be outlined in Section 3.3. The design inputs for this present work are specific to this project and will likely be different for other studies or projects. However, the method presented here is applicable to more than just the design point used for this project, specifically it is applicable to any case where the specified turbine thermodynamic design inputs are as follows:

- Output Power, $\dot{W}_{t}$

- Mass Flow Rate, $\dot{m}$

- Inlet Stagnation Temperature, $T_{01}$

- Inlet Stagnation Pressure, $P_{01}$

- Estimated Total-Total Isentropic Efficiency, $\eta_{t t}$

- Shaft Rotational Velocity, $R P M$

The value of estimated efficiency, $\eta_{t t}$, is a result of the cycle design and initial turbomachinery evaluation. If possible, it would be beneficial to iterate upon this estimated efficiency with better estimates from further turbine design, but this iteration was not completed due to time constraints of the project.

The turbine inlet total enthalpy, $h_{01}$, and entropy, $s_{01}$, are determined from the turbine inlet total conditions. REFPROP (as discussed in Section 2.4) was used within the preliminary design code for this project, but other equations of state (EoS) or fluid property tools could be used as well. The output power and mass flow rate define the required enthalpy drop in the turbine according to Equation 2.9, allowing for the turbine outlet total enthalpy, $h_{05}$, to be determined with Equation 3.2. Then, the ideal outlet total enthalpy, $h_{05 s s}$, may be determined with the estimated total-total efficiency, allowing for the outlet total pressure and temperature, $P_{05}$ and $T_{05}$, to be determined. With this inlet and outlet state point information, the turbine 
may be designed. Figure 3.1 (reproduced from Figure 2.6) indicates the station numbering used for the present turbine design methods.

$$
\begin{aligned}
{\left[h_{01}, s_{01}\right] } & =\operatorname{EoS}\left(T_{01}, P_{01}\right) \\
h_{05} & =h_{01}-\frac{\dot{W}_{t}}{\dot{m}} \\
h_{05 s s} & =\frac{h_{05}-h_{01}}{\eta_{t t}}+h_{01} \\
P_{05} & =\operatorname{EoS}\left(h_{05 s s}, s_{01}\right) \\
T_{05} & =\operatorname{EoS}\left(P_{05}, h_{05}\right)
\end{aligned}
$$
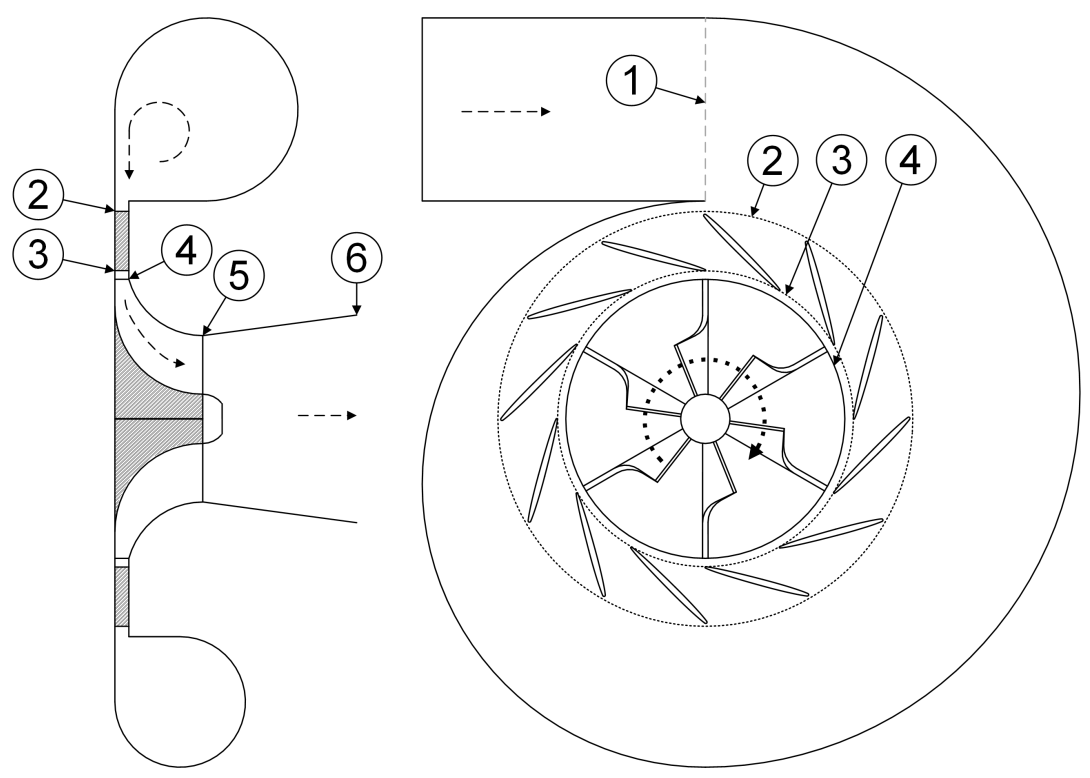

Figure 3.1: Radial turbine components and station locations for sizing.

\subsubsection{Rotor One-Dimensional Design Methods}

Ignoring frictional effects, the rotor is where the work is done in the turbine, so this is where the design is most critical and where the design process begins. The main outcomes of the one-dimensional rotor design are the inlet and outlet geometry (Figure 2.8) and velocity triangles (Figure 2.9) which have been introduced in Section 2.6. This rotor design process 
is unique because the $R P M$ is an input, whereas it is usually solved for as a design output. The $R P M$ was selected at an earlier stage in the overall project by a colleague. The $R P M$ selection was based on tradeoffs between turbomachinery performance because the proposed plant will have the compressor and turbine on the same shaft. The process presented here has been implemented in MATLAB, allowing for use and development in the future as needed. The rotor passage geometry and velocity triangles, Figure 2.8 and Figure 2.9, are presented again here as Figure 3.2 and Figure 3.3.

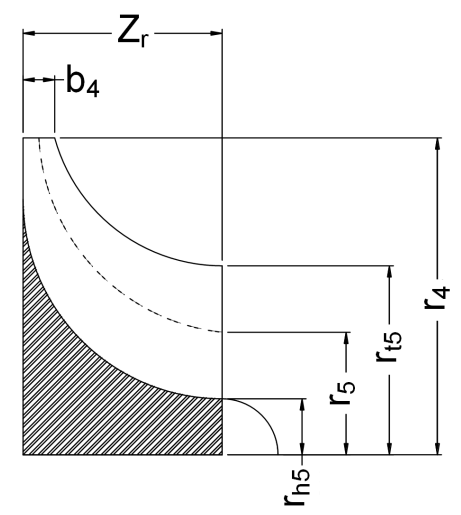

Figure 3.2: Radial turbine rotor sizing dimensions.

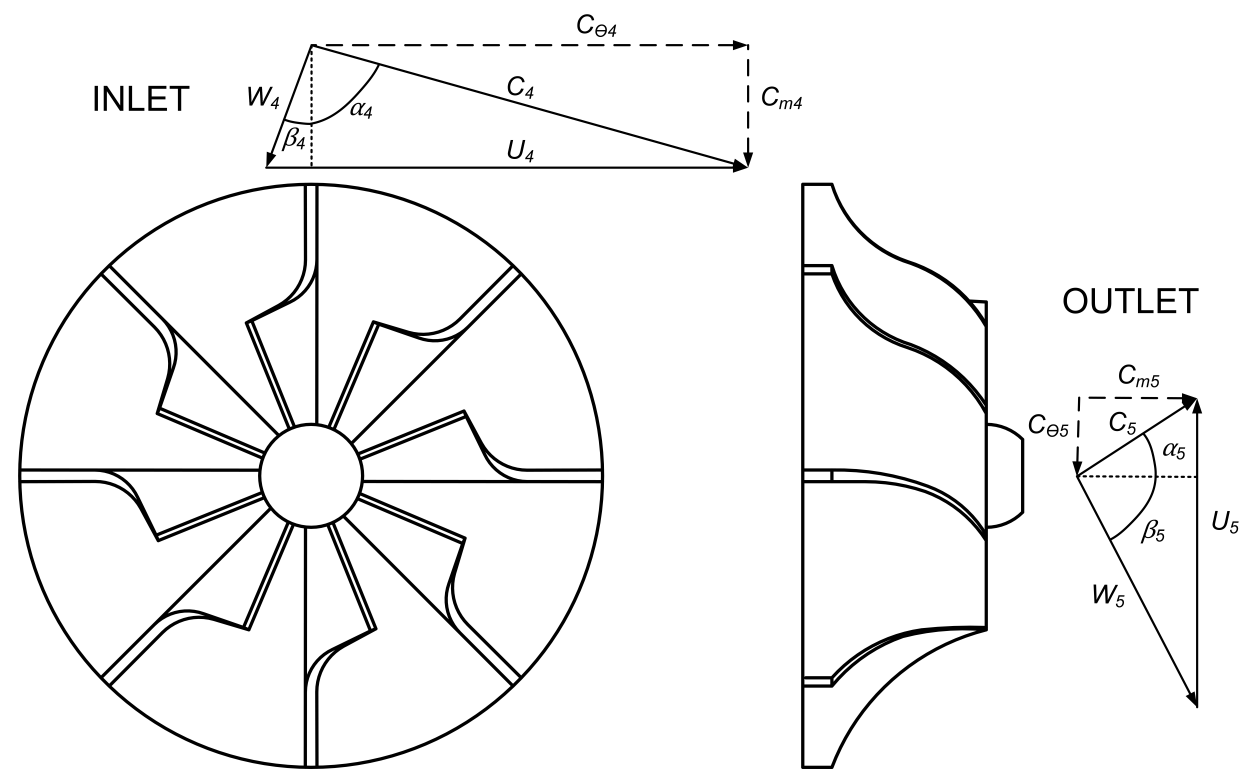

Figure 3.3: Radial turbine rotor example velocity diagrams. 


\section{Rotor Inlet Design}

In addition to the thermodynamic design inputs previously presented for the turbine, recommendations from literature are used to complete the rotor design, beginning with the inlet velocity triangle (Figure 3.4). The inlet relative flow angle, $\beta_{4}$, is one of these recommendations. Radial turbine rotor blades are usually radial at the inlet in order to reduce stress due to bending (Dixon \& Hall, 2013). One would think that the relative flow angle should be such that the flow aligns with the radial blades. However, it has been shown that for optimum efficiency, $\beta_{4}$ may range from $-20^{\circ}$ to $-40^{\circ}$ (Dixon \& Hall, 2013).

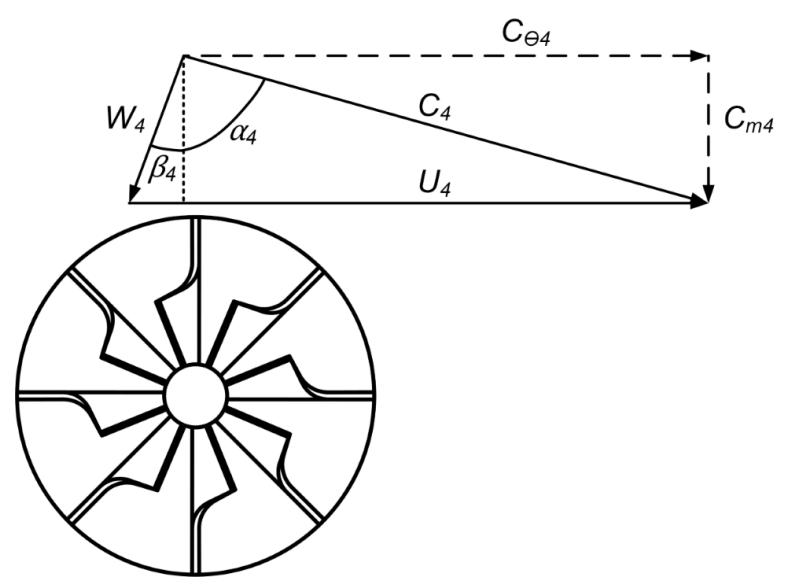

Figure 3.4: Rotor inlet velocity triangle.

The Work Coefficient, Equation 3.6, is specified as an input to fully define the inlet velocity triangle. The optimum value of the Work Coefficient lies between 0.8 and 1.0 as shown in Figure 2.10. Specifying a Work Coefficient as an input determines the inlet blade speed from the specified enthalpy drop. It is assumed that the inlet volute and nozzles will have only negligible losses, so the total pressure conditions at the rotor inlet are assumed to be equal to the turbine inlet total pressure conditions. Therefore, the rotor enthalpy drop, $\Delta h_{0}$, is specified as Equation 3.11, where heat transfer is neglected to equate $h_{04}$ to $h_{01}$. Now, the blade speed may be solved for as in Equation 3.12. For a certain state, $s_{0}$ is written instead of simply $s$ to indicate that the entropy value was obtained based on total conditions instead of static conditions, but the static and total entropy are still the same, $s_{0}=s$, because going 
from static to total (stagnation) is isentropic.

$$
\begin{aligned}
\Psi & =\frac{\Delta h_{0}}{U_{4}^{2}} \\
T_{04} & \approx T_{01} \\
P_{04} & \approx P_{01} \\
s_{04} & \approx s_{01} \\
h_{04} & \approx h_{01} \\
\Delta h_{0} & =h_{05}-h_{04} \approx h_{05}-h_{01} \\
U_{4} & =\sqrt{\frac{\Delta h_{0}}{\Psi}}
\end{aligned}
$$

With the blade speed and Work Coefficient, $\Psi$, the inlet tangential velocity, $C_{\theta 4}$, is calculated from Equation 3.13. The specified relative flow angle, $\beta_{4}$, then determines the meridional velocity, $C_{m 4}$, and the relative velocity, $W_{4}$. Finally, combining the absolute tangential and meridional components, the absolute velocity, $C_{4}$, and the absolute flow angle, $\alpha_{4}$, are found in Equation 3.16 and Equation 3.17.

$$
\begin{aligned}
C_{\theta 4} & =\Psi U_{4} \\
C_{m 4} & =\frac{U_{4}-C_{\theta 4}}{\tan \left(-\beta_{4}\right)} \\
W_{4} & =\frac{C_{m 4}}{\cos \left(\beta_{4}\right)} \\
C_{4} & =\sqrt{C_{m 4}^{2}+C_{\theta 4}^{2}} \\
\alpha_{4} & =\tan ^{-1}\left(\frac{C_{\theta 4}}{C_{m 4}}\right)
\end{aligned}
$$

The inlet velocity triangle is fully defined with the above calculations. The definition of the rotor inlet geometry follows. The absolute velocity, density, and mass flow rate determine the required inlet flow area, $A_{4}$, according to continuity. The static density, $\rho_{4}$, is determined 
through a total to static conversion (Equation 3.18) and an equation of state property lookup.

$$
\begin{aligned}
& h_{4}=h_{04}-\frac{1}{2} C_{4}^{2} \\
& \rho_{4}=\operatorname{EoS}\left(h_{4}, s_{04}\right) \\
& A_{4}=\frac{\dot{m}}{\rho_{4} C_{m 4}}
\end{aligned}
$$

The inlet area takes the form of a cylindrical surface meaning that the area (Equation 3.21) may be defined in terms of the inlet radius, $r_{4}$, and blade height, $b_{4}$. At this step, some designers choose to account for the blade blockage by increasing the required annulus area (White, 2015), others simply design the velocity triangles just outside of the blade passage (Aungier, 2006; Dixon \& Hall, 2013). The presented method does not account for the blade blockage and thus means that the inlet velocity triangle is defined just before the blades at the inlet. The area equation may be rearranged to solve for the required inlet radius (Equation 3.22). At this point, the blade height is unknown, so an initial guess may be used. The inlet radius and blade speed define the angular velocity which can be converted into a $R P M$. An iterative procedure or root finding method is used through Equation 3.12 to Equation 3.24 until the obtained value of $R P M$ matches the specified one by iterating or solving for the blade height, $b_{4}$. When the required blade height is found, the corresponding velocity triangle and rotor inlet size are defined.

$$
\begin{aligned}
A_{4} & =2 \pi r_{4} b_{4} \\
r_{4} & =\sqrt{\frac{A_{4}}{2 \pi \frac{b_{4}}{r_{4}}}} \\
\omega & =\frac{U_{4}}{r_{4}} \\
R P M & =\omega \frac{30}{\pi}
\end{aligned}
$$




\section{Rotor Outlet Design}

The rotor outlet design again uses literature recommendations to define the velocity triangle (Figure 3.5) and the basic sizing. The Flow Coefficient, $\Phi=C_{m 5} / U_{4}$, is a non-dimensional parameter specified as an input and used to define the outlet flow, with optimal values between 0.2 and 0.3 as shown in Figure 2.10. The absolute velocity at the outlet is commonly designed to be axial to avoid losses due to swirl in the diffuser and piping downstream (Whitfield, 1989). This means that the absolute outlet velocity is equal to the meridional velocity determined by the Flow Coefficient (Equation 3.25).

$$
C_{5}=C_{m 5}=\Phi U_{4}
$$

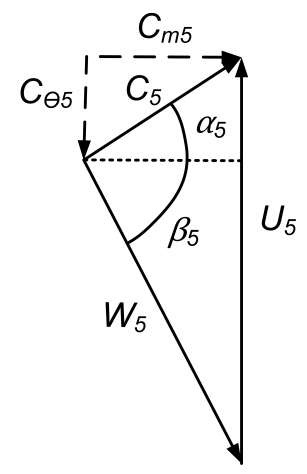

Figure 3.5: Rotor outlet velocity triangle.

The zero-swirl outlet velocity triangle has less components than the inlet velocity triangle, but the process of defining each component is somewhat similar. With the absolute velocity defined, the area required to pass the specified mass flow rate may be determined from continuity. The static enthalpy, $h_{5}$, is again used to find the static density, $\rho_{5}$, used for the area calculation.

$$
\begin{aligned}
h_{5} & =h_{05}-\frac{1}{2} C_{5}^{2} \\
s_{05} & =\operatorname{EoS}\left(T_{05}, P_{05}\right) \\
\rho_{5} & =\operatorname{EoS}\left(h_{5}, s_{05}\right) \\
A_{5} & =\frac{\dot{m}}{\rho_{5} C_{5}}
\end{aligned}
$$


Aungier (2006) suggests that a suitable ratio of the outlet hub radius to the inlet radius is about 0.185 . With this recommendation and geometric relations, the annular outlet area is defined by the outlet hub and tip radii $\left(r_{h 5}\right.$ and $\left.r_{t 5}\right)$ as determined by Equation 3.30 and Equation 3.31. Again, the blade blockage is not taken into account in the presented method. Recall from the literature review that the root mean square radius, $r_{5}$ (Equation 3.32), is used for the outlet meanline design.

$$
\begin{aligned}
r_{h 5} & =0.185 r_{4} \\
r_{t 5} & =\sqrt{r_{h 5^{2}-A_{5} / \pi}} \\
r_{5} & =\sqrt{\frac{r_{h 5^{2}+r_{t 5}^{2}}}{2}}
\end{aligned}
$$

To complete the outlet velocity triangle, the angular velocity, $\omega$, along with the radius defines the blade speed, $U_{5}$ (Equation 3.33). Finally, the relative flow magnitude, $W_{5}$, and angle, $\beta_{5}$, complete the velocity triangle.

$$
\begin{aligned}
U_{5} & =r_{5} \omega \\
\beta_{5} & =-\tan ^{-1}\left(\frac{U_{5}}{C_{5}}\right) \\
W_{5} & =\frac{C_{5}}{\cos \left(\beta_{5}\right)}
\end{aligned}
$$

\section{Rotor Blade Definitions}

Recommendations for the count, thickness, and metal angle of the rotor blades are found in literature. Dixon \& Hall (2013) present a good discussion on the criterion for the minimum number of rotor blades, including the need to avoid reverse flow at the inlet due to the use of too few blades. Dixon \& Hall (2013) also highlight that the number of blades is usually minimized to reduce the wetted area causing frictional losses, to reduce the weight and inertia, and to reduce the flow blockage at the hub of the rotor exit. Aungier (2006) suggest that the blade number relationship presented by Rohlik (1968) (Equation 3.36) provides a good balance 
between too few and too many blades.

$$
N_{R}=12+0.03\left(\alpha_{4}-57\right)^{2}
$$

The blade thickness is specified with relationships suggested by Aungier (2006). The thickness at the inlet, $t_{b 4}$, and outlet, $t_{b 5}$, are related to the rotor inlet radius by Equation 3.37 and Equation 3.38. Aungier (2006) recommends a streamwise thickness distribution for the blades in which the blade maintains the inlet thickness until the last $10 \%$ of the streamwise distance to the trailing edge, then it is tapered to the outlet thickness. A uniform spanwise thickness for the whole blade was applied for this project.

$$
\begin{aligned}
& t_{b 4}=0.04 r_{4} \\
& t_{b 5}=0.02 r_{4}
\end{aligned}
$$

The rotor blade metal angle at the inlet is radial, $\beta_{4^{\prime}}=0^{\circ}$, for structural considerations Dixon \& Hall (2013). The outlet metal angle on the other hand requires an understanding of the deviation off the trailing edge because an outlet flow with no swirl is desired. Characterizing the deviation at this stage is challenging due to the three-dimensional nature of the flow. For this reason, a preliminary estimate of $\beta_{5^{\prime}}=\beta_{5}$ is used for the one-dimensional design and any deviation is corrected in the CFD design stage by adjusting the metal angle accordingly.

Finally, Aungier (2006) suggests that the axial length of the rotor be determined in terms of the outlet radii as:

$$
L_{R}=1.5\left(r_{t 5}-r_{h 5}\right)
$$

\section{Rotor Design Evaluation}

With the basic rotor size and velocity triangles established, the design should be reviewed for feasibility based on empirical relations. These relations may be geometric ratios, velocity ratios, or other guidelines. Aungier (2006) gathered the following recommendations:

- The rotor outlet to inlet radius ratio, $r_{t 5} / r_{4}$, should conservatively be $\leq 0.7$ 
- The Meridional Velocity Ratio, $C_{m 5} / C_{m 4}$, should be between 1 and 1.5

- The stage reaction, $R=\left(h_{4}-h_{5}\right) /\left(h_{04}-h_{05}\right)$, should be between 0.45 and 0.65

- The specific speed, $N_{s}$, should be between 0.45 and 0.75 , where $N_{s}=\frac{\omega Q^{\frac{1}{2}}}{\left(\Delta h_{0 s s}\right)^{\frac{3}{4}}}$

These ranges were used to evaluate the obtained design in terms of recommendations not specified as inputs in Section 3.3.2.

Another important evaluation of the rotor preliminary design is to ensure that the Mach numbers remain subsonic, $M<0.9$. The relative Mach number at the rotor outlet tip and the absolute velocity at the rotor inlet are monitored to avoid supersonic conditions and losses due to shocks (Japikse \& Baines, 1994). The static speed of sound values are obtained from the static enthalpy at the rotor inlet and outlet.

$$
\begin{aligned}
a_{4} & =\operatorname{EoS}\left(h_{4}, s_{04}\right) \\
\mathrm{Ma}_{4} & =\frac{C_{4}}{a_{4}} \\
a_{5} & =\operatorname{EoS}\left(h_{5}, s_{05}\right) \\
U_{t 5} & =r_{t 5} \omega \\
\beta_{t 5} & =-\tan ^{-1}\left(\frac{U_{t 5}}{C_{5}}\right) \\
W_{t 5} & =\frac{C_{5}}{\cos \left(\beta_{t 5}\right)} \\
\mathrm{Ma}_{t 5, r e l} & =\frac{W_{t 5}}{a_{5}}
\end{aligned}
$$

\subsubsection{Nozzle One-Dimensional Design Methods}

The nozzle design follows the rotor design as the rotor inlet sizing and velocity triangle information define the nozzle requirements. The nozzles, as part of the stator, impart swirl to the flow (if necessary) and accelerate it. As stated previously, in the preliminary design stage, the working fluid total conditions are simply assumed to be constant through the stator. The nozzle design methods presented in this section will cover the geometric passage sizing, the initial blade geometry, and the blade count. 
First, a vaneless passage is included between the nozzle row outlet and rotor inlet, from station 3 to 4 in Figure 2.6. This vaneless passage is included to allow the wake from the nozzle row to mix closer to uniformity before the flow reaches the rotor inlet. Watanabe et al. (1971) is commonly cited for his recommendation regarding the vaneless passage (Equation 3.47). From this relationship, the nozzle outlet radius, $r_{3}$, may be calculated.

$$
r_{3} / r_{4}=1+2 b_{4} \sin \left(\alpha_{4}\right) / r_{4}
$$

Next, conservation of angular momentum is applied to determine the tangential velocity at $r_{3}$ (Equation 3.48). Then, the meridional velocity at station 3 is found by applying continuity. Like the rotor, the meridional velocities in this procedure will be defined outside of the blades. It was chosen to hold the blade height from the rotor inlet constant through the nozzle row, $b_{3}=b_{2}=b_{4}=b$. An iterative approach is taken to overcome the fact that the density at station 3 is unknown at this point. An initial guess of $\rho_{3}=\rho_{4}$ is used and then the steps from Equation 3.49 through Equation 3.52 are iterated until the density (and therefore meridional velocity) converges.

$$
\begin{aligned}
C_{\theta 3} & =C_{\theta 4}\left(\frac{r_{4}}{r_{3}}\right) \\
C_{m 3} & =\frac{\dot{m}}{2 \pi r_{3} b \rho_{3}} \\
C_{3} & =\sqrt{C_{\theta 3}^{2}+C_{m 3}^{2}} \\
h_{3} & =h_{03}-0.5 C_{3}^{2} \\
\rho_{3} & =\operatorname{EoS}\left(h_{3}, s_{03}\right) \\
\alpha_{3} & =\tan ^{-1}\left(\frac{C_{\theta 3}}{C_{m 3}}\right)
\end{aligned}
$$

The number of nozzle blades, $N_{N}$, nozzle row inlet radius, $r_{2}$, and the nozzle blade pitch to chord ratio, $s_{3} / c_{N}$, are key nozzle design parameters. In the presented method, $s_{3} / c_{N}$ is used as a design input, and $N_{N}$ and $r_{2}$ are design outputs. Aungier (2006) recommends a value of $s_{3} / c_{N}=0.75$, whereas White (2015) indicated that lower values were required for smaller 
machines (using $s_{3} / c_{N}=0.5$ for their Organic Rankine Cycle turbine). Aungier (2006) also recommends that the nozzle row inlet to outlet radius ratio, $r_{2} / r_{3}$, should be between 1.1 and 1.7. The specified $s_{3} / c_{N}$ and resulting $r_{2} / r_{3}$ should fall within these limits. The following procedure determines the $r_{2}$ and $N_{N}$.

The blade pitch, $s_{3}$, is found through Equation 3.54. This requires an initial guess for $N_{N}$ and the number of rotor blades, $N_{R}$, may be a good first guess because there are usually more nozzle blades than rotor blades. From the specified $s_{3} / c_{N}$ ratio, the desired chord length, $c_{N}$, may be calculated from $s_{3}$.

$$
s_{3}=2 \pi r_{3} / N_{N}
$$

With the chord length, ratios presented by Aungier (2006) may be used to determine the blade thickness distribution based on Figure 2.11. The leading edge thickness, $t_{b 2}$, trailing edge thickness, $t_{b 3}$, maximum thickness, $t_{\max }$, and the chordwise location of the maximum blade thickness, $d$, are specified based on the chord, $c_{N}$, in Equations 3.55 through 3.58. The ratios suggested by Aungier (2006) are as follows:

$$
\begin{aligned}
\frac{t_{b 2}}{c_{N}} & =0.025 \\
\frac{t_{b 3}}{c_{N}} & =0.012 \\
\frac{t_{\max }}{c_{N}} & =0.06 \\
\frac{d}{c_{N}} & =0.4
\end{aligned}
$$

Next, the required nozzle outlet metal angle, $\beta_{3^{\prime}}$, is defined based on the throat width, $o_{t h}$, required to achieve the specified blade pitch and flow angle. The required throat width is specified by Equation 3.59, where the flow angle at the throat, $\alpha_{t h}$, is found by Equation 3.60. $r_{t h}$ is the mean radius of the throat as shown in Figure 3.6. A root finding method may be applied to find the value of $\beta_{3^{\prime}}$ that achieves the required throat width. The method solves Equation 3.61, where $d_{t h}$ is the shortest distance from the trailing edge of one blade to the line defined by the chord of the next blade for uncambered blades as shown in Figure 3.6. 
One maximum blade thickness, $t_{\max }$, value is subtracted from $d_{t h}$ to account for the blade thickness as an estimation. The equation of the chord line may be defined as Equation 3.62 and the trailing edge point of the adjacent blade may be defined as Equation 3.63. Then, $d_{t} h$ is found mathematically as shown in Equation 3.64.

$$
\begin{aligned}
o_{t h} & =s_{3} \cos \left(\alpha_{t h}\right) \\
r_{t h} \alpha_{t h} & =r_{3} \alpha_{3} \\
o_{t h} & =d_{t h}-t_{\max } \\
0 & =\tan \left(90-\beta_{3^{\prime}}\right) x-y+r_{3}=a x+b y+c \\
P_{t e} & =\left(r_{3} \sin \left(360 / N_{N}\right), r_{3} \cos \left(360 / N_{N}\right)\right)=\left(x_{0}, y_{0}\right) \\
d_{t h} & =\frac{\left|a x_{0}+b y_{0}+c\right|}{\sqrt{a^{2}+b^{2}}}
\end{aligned}
$$

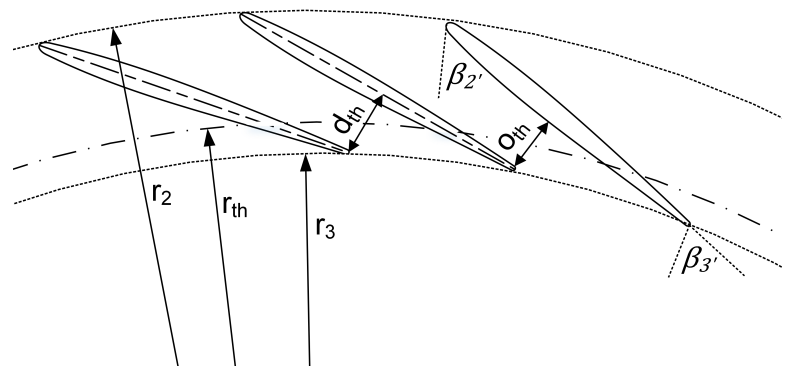

Figure 3.6: Illustration for nozzle throat dimensions (Adapted from Aungier, 2006).

The radius at the nozzle inlet, $r_{2}$, corresponding to the specified $r_{3}, \beta_{3^{\prime}}$, and $c_{N}$ is determined by the endpoint of a line, with a length of $c_{N}$, originating from a circle with radius $r_{3}$ and extending with angle $\beta_{3^{\prime}}$ from the meridional. This is shown in Figure 3.7.

The metal angle at the nozzle inlet for the uncambered blade may be found from Equation 3.65. Aungier (2006) also presents correlations relating the optimum nozzle incidence angle, $i^{*}$ (Equation 3.66), and flow angle, $\alpha^{*}$ (Equation 3.67), to the nozzle blade specifications. It is assumed that the volute upstream of the nozzle will deliver the flow to the nozzles 


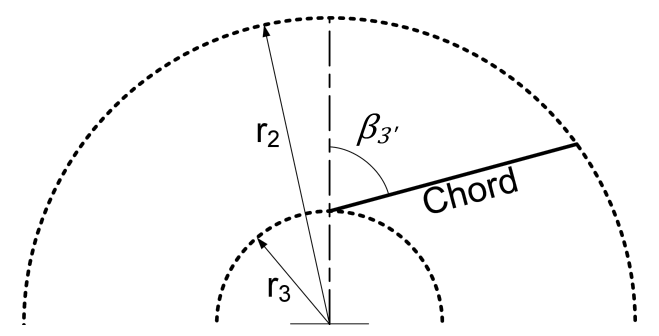

Figure 3.7: Illustration of nozzle chord in relation to nozzle inlet radii, outlet radii, and outlet setting angle.

at this optimum incidence, so $\alpha_{2}=\alpha^{*}$.

$$
\begin{aligned}
\beta_{2^{\prime}} & =\sin ^{-1}\left(\sin \left(\beta_{3^{\prime}}\right) \frac{r_{3}}{r_{2}}\right) \\
i^{*} & =\left[3.6 \sqrt{\frac{10 t_{b 2}}{c_{N}}}+\frac{\left|\beta_{3^{\prime}}-\beta_{2^{\prime}}\right|}{3.4}\right] \sqrt{\frac{c_{N}}{s_{3}}}-\frac{\left|\beta_{3^{\prime}}-\beta_{2^{\prime}}\right|}{2} \\
\alpha^{*} & =\beta_{2^{\prime}}-i^{*} \operatorname{sign}\left(\beta_{3^{\prime}}-\beta_{2^{\prime}}\right)
\end{aligned}
$$

The nozzle inlet velocities are based on the geometry. To determine these flow velocities, first, an initial value of the meridional velocity, $C_{m 2}$, is estimated to obtain an estimate for the static density, $\rho_{2}$. Aungier (2006) suggests that this initial estimate may come from $r_{2} C_{m 2} \approx r_{3} C_{m 3}$. The passage area is known to be equal to the cylindrical surface defined by $r_{2}$ and $b_{4}$. An iterative approach or root finding method may be applied to find the meridional velocity that corresponds to the flow angle, $\alpha_{2}$, and passage area. The corresponding process is as follows, where an iterative approach is used until the value of $C_{m 2}$ converges:

$$
\begin{aligned}
C_{\theta 2} & =C_{m 2} \tan \left(\alpha_{2}\right) \\
C_{2} & =\sqrt{{C_{\theta 2}}^{2}+C_{m 2}^{2}} \\
h_{2} & =h_{02}-0.5 C_{2}^{2} \\
\rho_{2} & =\operatorname{EoS}\left(h_{2}, s_{02}\right) \\
C_{m 2} & =\frac{\dot{m}}{2 \pi r_{2} b \rho_{2}}
\end{aligned}
$$


In Equation 3.54, an initial estimate for $N_{N}$ was used. The number of nozzle blades required is based on a reasonable limit for the loading of each blade. Aungier (2006) suggests that the maximum blade-to-blade velocity difference may be estimated by Equation 3.73 and that a reasonable blade loading may be obtained with the limit in Equation 3.74. If this limit is not satisfied, then $N_{N}$ is increased and the process beginning at Equation 3.54 is repeated.

$$
\begin{aligned}
& (\Delta C)_{\text {max }}=\frac{4 \pi\left(r_{3} C_{\theta 3}-r_{2} C_{\theta 2}\right)}{c_{N} N_{N}} \\
& (\Delta C)_{\text {max }} \leq \frac{\left(C_{2}+C_{3}\right)}{2}
\end{aligned}
$$

\subsubsection{Volute One-Dimensional Design Methods}

Upstream of the nozzle row, is the initial turbine stator component, the inlet volute. The scroll volute acts to turn flow in a pipe from the tangential direction radially inwards towards the rotor's axis of rotation. As part of the stator, the volute acts to impart swirl to the flow. The swirl is already present in the flow because it enters tangentially, so the volute acts mostly to distribute this swirl circumferentially. A key design goal of the volute is the uniform distribution of the flow around the circumference. The total conditions through the volute are again assumed to be constant for the preliminary design. The scroll volute is shown in Figure 3.8 (reproduced from Figure 2.12), with both the radial locations and azimuthal cross-section shown. An external volute with an aspect ratio, $R=A^{\prime} / B^{\prime}$, of 1 as described by Aungier (2006) has been used in this project.

Like the nozzle row design, the volute design begins with a vaneless passage between it and the next downstream component, the nozzles in this case. The ratio between the volute exit radius, $r_{2 \mathrm{v}}$, and the nozzle inlet radius, $r_{2}$, was assumed to be 1.05 . This vaneless passage should be long enough to allow the flow to reattach after turning a corner radially inward from the volute circular cross-section. The height for this vaneless passage is constant with the blade height of the nozzles and rotor inlet, $b=b_{4}$. 


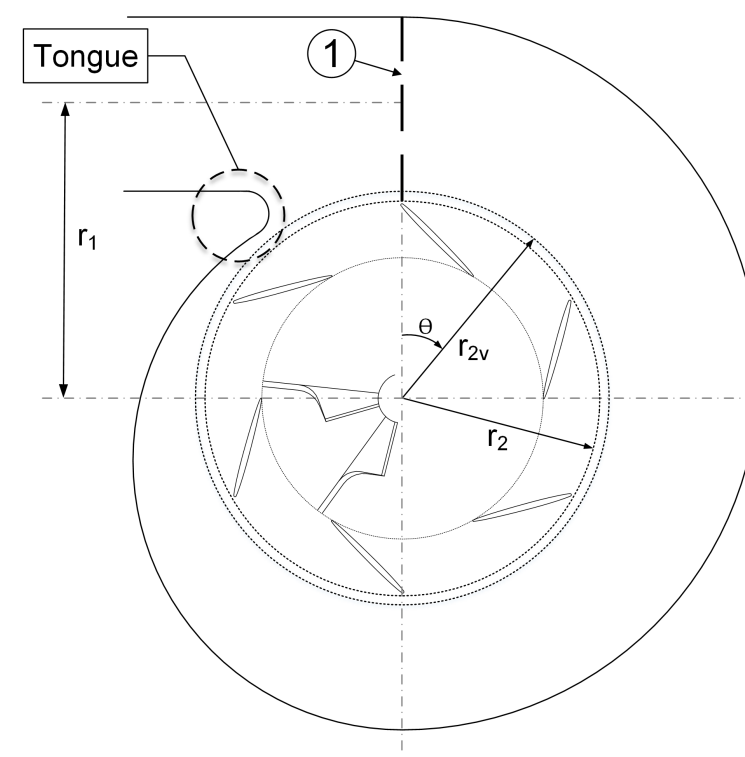

(a) Volute radial locations.

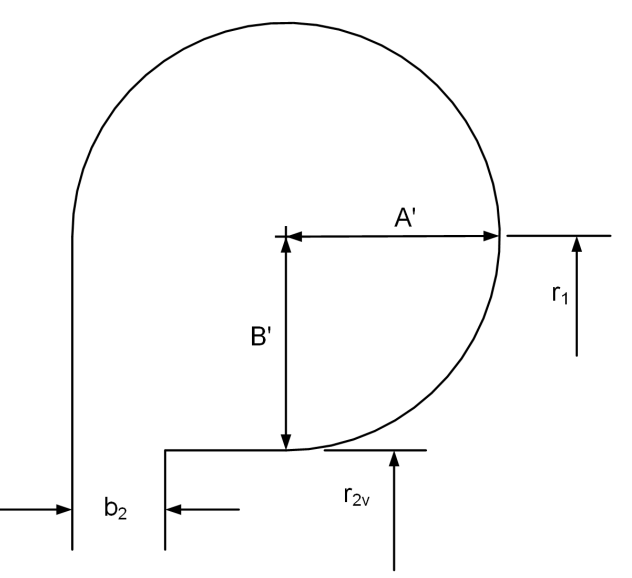

(b) Volute cross section.

Figure 3.8: General radial turbine scroll volute dimensions.

The velocities at $r_{2 \mathrm{v}}$ are determined in a similar fashion as the velocities at station 3 for the nozzle row. The tangential velocity is determined by Equation 3.75.

$$
C_{\theta 2 \mathrm{v}}=C_{\theta 2}\left(\frac{r_{2}}{r_{2 \mathrm{v}}}\right)
$$

Conservation of angular momentum is applied through the volute so that the velocity at the volute inlet, station 1 in Figure 2.12, may be determined through Equation 3.76. $S_{c}$ is the Swirl Coefficient, a non-dimensional correction factor suggested by Japikse \& Baines (1994) to range from 0.85 to 0.95 to account for wall friction effects through the volute in Equation 3.76. The value of $C_{1}$ increases proportional to $1 / S_{c}$, resulting in a smaller required cross-section for continuity. Referring to Figure 2.12, the required cross-section area, $A_{1}$, cross-section radii, $A^{\prime}$ and $B^{\prime}$, and corresponding radius, $r_{1}$, are determined iteratively through Equation 3.76 to Equation 3.82 until $r_{1}$ converges. This sizing process assumes that the turbine delivery velocity (upstream of station 1) is not restricted by piping design or can be achieved by tapering the 
inlet piping accordingly.

$$
\begin{aligned}
C_{1} & =C_{\theta 2 \mathrm{v}}\left(\frac{r_{2 \mathrm{v}}}{S_{c} r_{1}}\right) \\
h_{1} & =h_{01}-0.5 C_{1}^{2} \\
\rho_{1} & =\operatorname{EoS}\left(h_{1}, s_{01}\right) \\
A_{1} & =\frac{\dot{m}}{\rho_{1} C_{1}} \\
A^{\prime} & =\sqrt{\frac{A_{1} R}{\frac{3}{4} \pi+1}} \\
B^{\prime} & =\frac{A}{R} \\
r_{1} & =r_{2 \mathrm{v}}+B
\end{aligned}
$$

The cross-section and radial geometry may similarly be determined for the whole volute as a function of azimuth angle, $\theta$. Conservation of angular momentum is applied again such that $r C_{\theta}$ is constant and for a uniform distribution of mass flow, the mass flow through any cross section at a specific azimuth location is $\dot{m}_{\theta}=\dot{m}\left(1-\frac{\theta}{2 \pi}\right)$ (Japikse \& Baines, 1994). The determination of the cross-section geometry and radii follows in a similar manner as before, iterating until $r_{\theta}$ converges. Solving for these values completes the scroll volute onedimensional design.

$$
\begin{aligned}
C_{\theta} & =C_{1}\left(\frac{r_{1}}{r_{\theta}}\right) \\
h_{\theta} & =h_{0 \theta}-0.5 C_{\theta}^{2} \\
\rho_{\theta} & =\operatorname{EoS}\left(h_{\theta}, s_{01}\right) \\
A_{\theta} & =\frac{\dot{m}_{\theta}}{\rho_{\theta} C_{\theta}} \\
A_{\theta}^{\prime} & =\sqrt{\frac{A_{\theta} R}{\frac{3}{4} \pi+1}} \\
B_{\theta}^{\prime} & =\frac{A^{\prime}}{A} \\
r_{\theta} & =r_{2 \mathrm{v}}+B_{\theta}
\end{aligned}
$$




\section{3 ${\mathrm{S}-\mathrm{CO}_{2}}$ Radial Turbine One-Dimensional Design}

In this section, the one-dimensional design methodology presented in the prior section is applied to the specific design case of this project. The design specifications for the turbine are presented in the following subsection, followed by the resulting radial turbine rotor, nozzle row, and inlet volute preliminary designs.

\subsubsection{Turbine Design Specifications}

The turbine designed for this project was part of a larger project evaluating the feasibility of a S-CO2 power cycle. The cycle design is not within the scope of this work, so only the turbine design inputs are referenced in the present work. The goal of this turbine design process was to achieve a complete turbine aerodynamic design capable of meeting the required power output with an acceptable efficiency and then to create performance maps for system modelling of the complete cycle. The turbine design inputs for this project are presented in Table 3.1. The estimated efficiency value was obtained from initial estimates from a colleague, Mitch Kibsey, based on loss models and a similar sized turbine prior to the present author's work. The estimate of $86 \%$ may be conservative, but it was assumed that it would be better to have a low estimate and find out the machine overperforms than have a high estimate and a machine that underperforms.

Table 3.1: Turbine thermodynamic design inputs.

\begin{tabular}{lrrl}
\hline \hline Parameter & & Value & Units \\
\hline Power output & $\dot{W}_{t}$ & 14.72 & $\mathrm{MW}$ \\
Inlet total temperature & $T_{01}$ & 631.25 & $\mathrm{~K}$ \\
Inlet total pressure & $P_{01}$ & 23290 & $\mathrm{kPa}$ \\
Mass flow rate & $\dot{m}$ & 170 & $\mathrm{~kg} / \mathrm{s}$ \\
Estimated total-total isentropic efficiency & $\eta_{t t}$ & 86 & $\%$ \\
$R P M$ & & 21000 & $\mathrm{rpm}$ \\
\hline \hline
\end{tabular}




\subsubsection{Rotor One-Dimensional Design}

As stated previously, the turbine design process begins with the rotor, where the work is done. Along with the thermodynamic inputs specified in Table 3.1, the rotor design requires the specification of the Work Coefficient, $\Psi$, Flow Coefficient, $\Phi$, and inlet relative flow angle, $\beta_{4}$. Parametric studies were performed to understand the effect of these parameters on the rotor design and to arrive at acceptable initial values.

First, the variation of the Meridional Velocity Ratio, $\xi$ (Equation 3.90), with these variable parameters was investigated. Aungier (2006) recommends that the rotor be designed such that $\xi$ falls between 1.0 and 1.5, favouring values closer to 1 . Figure 3.9 shows the variation in $\xi$ with the variable parameters. Values of $\Psi$ above 0.95 were not investigated because they result in $\xi>1.5$ for all investigated $\beta_{4}$ values. $\Phi$ values up to 0.4 were included in the investigation because an interesting relationship was seen with the Rotor Relative Velocity Coefficient, $\phi_{R}$,

as will be discussed later. All three investigated $\beta_{4}$ values achieve the desired $1.0 \leq \xi \leq 1.5$. However, with $\beta_{4}=-40$, the desired range of $\xi$ is only achieved with low $\Psi$ values. From Equation 2.18, values of $\Psi$ closer to 1 are desired. For this reason, $\beta_{4}=-20$ can be seen to be better than $\beta_{4}=-30$ with the desired $\xi$ range at higher values of $\Psi$.

$$
\xi=\frac{C_{m 5}}{C_{m 4}}
$$

Next, the effect of these variable input parameters on the Rotor Relative Velocity Coefficient, $\phi_{R}$, was examined. $\phi_{R}$ is the ratio of the relative velocity at the rotor outlet, $W_{5}$, to the relative velocity at the outlet if the expansion through the rotor was isentropic, $W_{5 s}$ (Equation 3.91). Dixon \& Hall (2013) shows that $\phi_{R}$ is related to the losses through the rotor and suggests that $\phi_{R}$ falls between 0.7 and 0.85 for well-designed radial inflow turbines. When $\phi_{R}$ is close to 1 , the expansion through the rotor is closer to ideal and values above 1 indicate over-expansion (White, 2015). The Rotor Relative Velocity Coefficient was only seen to be dependent on the Work and Flow Coefficients and did not vary significantly with changes in the inlet relative flow angle. Figure 3.10 shows the variation in $\phi_{R}$ with $\Psi$ and $\Phi$ 

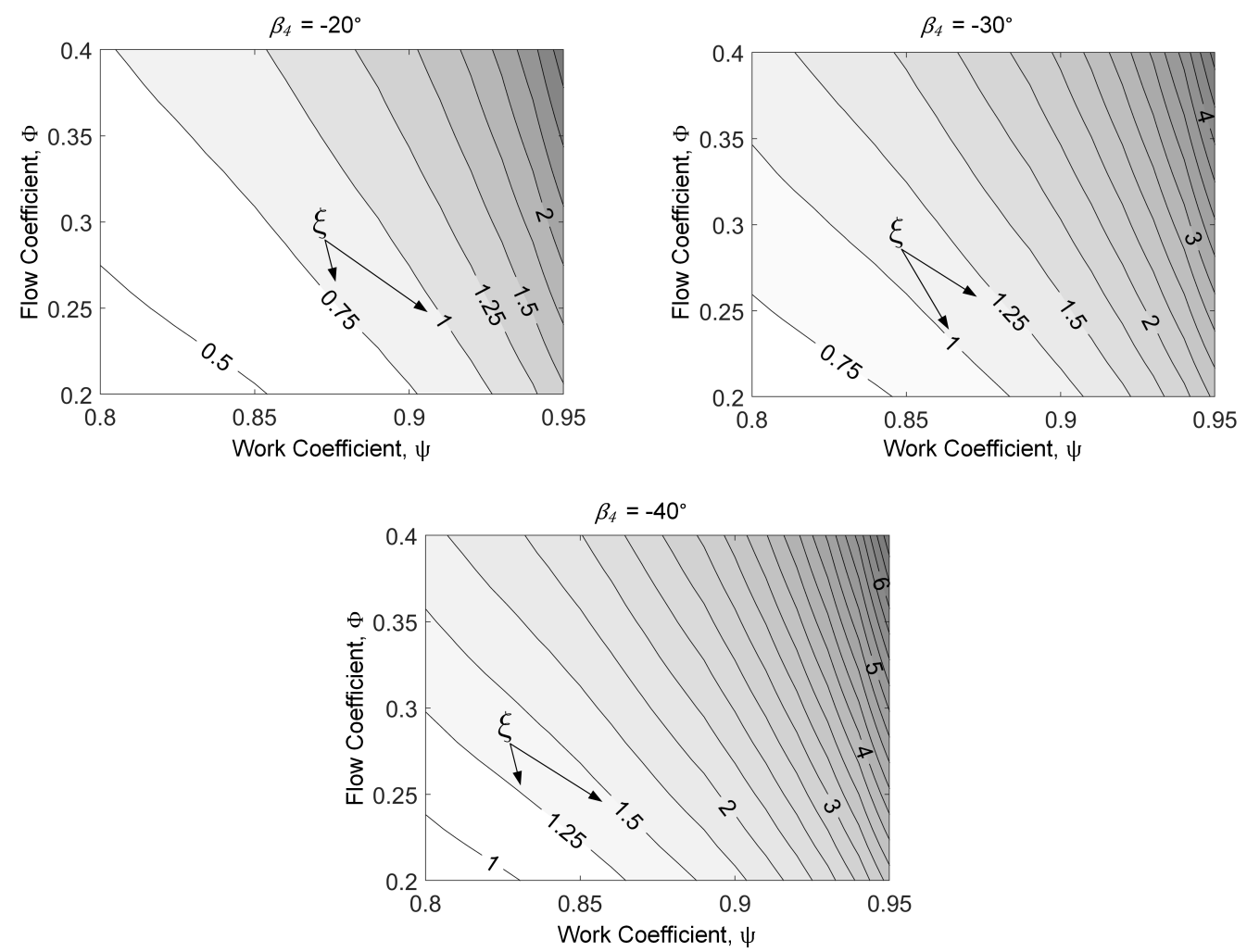

Figure 3.9: Variation in $\xi$ with $\Psi$ and $\Phi$ at different $\beta_{4}$ values.

indicating that the investigated values of Flow Coefficient and Work Coefficient can result in $\phi_{R}$ within $0.70-0.85$. Interestingly, $\phi_{R}$ is shown to be furthest from 1 as $\Phi$ is between 0.25 and 0.3 for the investigated range of $\Psi$, meaning that conservative values of $\Phi$ around $0.25-$ 0.30 may correspond to higher losses in this scenario. This phenomenon may be something to investigate in the future but was not investigated in the present work. The minimum value of $\phi_{R}$ for the investigated range or variable inputs remained above 0.70 , so it was deemed that successful designs were possible in the examined range.

$$
\phi_{R}=\frac{W_{5}}{W_{5 s}}
$$

where

$$
\begin{aligned}
I_{4} & =h_{4}+\frac{1}{2} C_{4}^{2}-U_{4} C_{\theta 4}=h_{4}+\frac{1}{2}\left(W_{4}^{2}-U_{4}^{2}\right) \\
I_{5} & =I_{4} \\
h_{5 s} & =\operatorname{EoS}\left(P_{5}, s_{4}\right)
\end{aligned}
$$




$$
W_{5 s}=\sqrt{2\left(I_{5}-h_{5 s}\right)+U_{5}^{2}}
$$

where $I$ is the rothalpy.

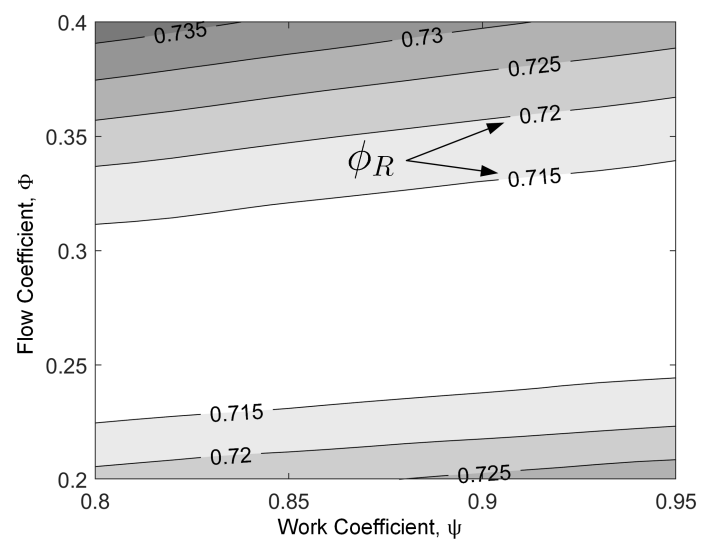

Figure 3.10: Variation in $\phi_{R}$ with $\Psi$ and $\Phi$.

The selected values for these variable rotor design inputs are listed in Table 3.2. A conservative Work Coefficient value of 0.89 was selected in the hopes that it would increase the chances of a successful first design. The Flow Coefficient was selected as 0.30 and $\beta_{4}$ was chosen to be $-20^{\circ}$ to obtain acceptable $\xi$ values at the chosen Work Coefficient.

Table 3.2: Rotor variable design inputs.

\begin{tabular}{lrr}
\hline \hline Parameter & & Value \\
\hline Work Coefficient & $\Psi$ & 0.89 \\
Flow Coefficient & $\Phi$ & 0.30 \\
Inlet relative flow angle & $\beta_{4}$ & $-20^{\circ}$ \\
\hline \hline
\end{tabular}

The meridional geometry of the final one-dimensional design is shown in Figure 3.11. The rotor tip and rotor hub diameters are only $284 \mathrm{~mm}$ and $50.0 \mathrm{~mm}$.

Key output parameters for the rotor design are included in Table 3.3. The design passes all checks presented in Section 3.2.1. The estimated $\eta_{t s}$ is low when compared to Figure 2.10 where the efficiencies plotted around $\Psi=0.89, \Phi=0.30$ are around $\eta_{t s}=0.86$. The low $\eta_{t s}$ value corresponds to the conservative estimated $\eta_{t t}$ value. This $\eta_{t s}$ difference has been left as future work as only one estimated $\eta_{t t}$ value was investigated because time limitations did not allow for iteration on the initial cycle specifications of the overall S-CO2 power cycle project. 


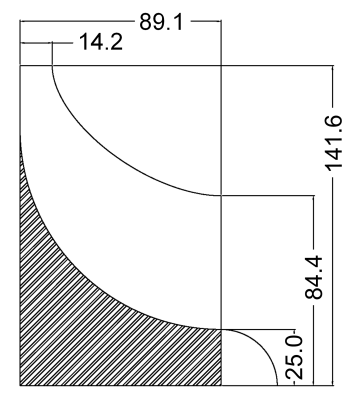

Figure 3.11: One-dimensional rotor meridional geometry (Dimensions in $\mathrm{mm}$ ).

Untapered blades were used for this first design iteration. In the future, it is recommended to taper the blade thickness from root to tip to reduce the centrifugal stresses.

Table 3.3: Rotor one-dimensional design output parameters.

\begin{tabular}{lcrr}
\hline \hline Parameter & & Value & Units \\
\hline Number of rotor blades & $N_{R}$ & 19 & \\
Rotor outlet to inlet radius ratio & $r_{t 5} / r_{4}$ & 0.60 & \\
Meridional Velocity Ratio & $C_{m 5} / C_{m 4}$ & 1.03 & \\
Stage reaction & $R$ & 0.56 & \\
Specific speed & $N_{s}$ & 0.51 & \\
Estimated rotor isentropic total-static efficiency & $\eta_{t s}$ & 82.5 & $\%$ \\
Inlet absolute flow angle & $\alpha_{4}$ & 71.9 & $\circ$ \\
Outlet hub relative flow angle & $\beta_{h 5}$ & -30.5 & $\circ$ \\
Outlet tip relative flow angle & $\beta_{t 5}$ & -63.3 & $\circ$ \\
Inlet absolute Mach number & $\mathrm{Ma}_{4}$ & 0.78 & \\
Outlet tip relative Mach number & $\mathrm{Ma}_{t 5}$ & 0.59 & \\
Rotor inlet blade thickness & $t_{b 4}$ & 3.00 & $\mathrm{~mm}$ \\
Rotor outlet blade thickness & $t_{b 5}$ & 2.83 & $\mathrm{~mm}$ \\
\hline \hline
\end{tabular}

The rotor one-dimensional design velocity triangles are shown in Figure 3.12. The inlet velocity triangle has a relative velocity, $W_{4}$, at the specified angle, $\beta_{4}$, in the opposite direction to the rotation. The outlet absolute velocity, $C_{5}$, leaves the rotor in the axial direction at $\alpha_{5}=0$ as desired. 


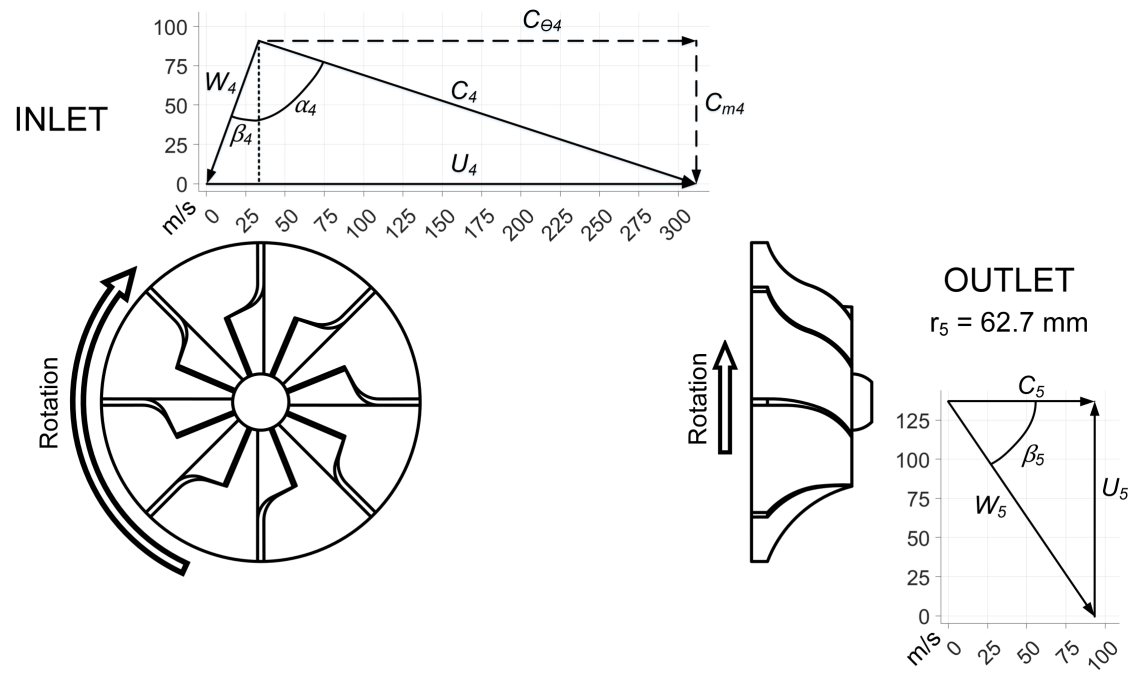

Figure 3.12: One-dimensional rotor velocity triangles (rotor geometry not to scale).

\subsubsection{Stator One-Dimensional Design}

The nozzle and volute designs did not include parametric studies like the rotor design did, so this section simply presents the resulting geometries. The methods presented in the methodology section were directly applied for the stator design. The nozzle row was designed with a blade pitch to chord ratio $s_{3} / c_{N}=0.6$ because larger values resulted in low inlet to outlet radius ratios. Figure 3.13 shows the geometry of the one-dimensional nozzle row and volute. The resulting nozzle radius ratio was satisfactory according to literature recommendations at 1.13 and other key nozzle one-dimensional design output parameters are presented in Table 3.4. The scroll volute was designed with $S_{c}=0.85$ as a first approximation.

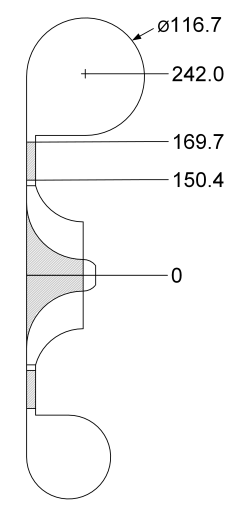

Figure 3.13: One-dimensional volute and rotor geometry (Dimensions in $\mathrm{mm}$ ). 
Table 3.4: Nozzle one-dimensional design output parameters.

\begin{tabular}{lcrl}
\hline \hline Parameter & & Value & Units \\
\hline Number of nozzle blades & $N_{N}$ & 35 & \\
Nozzle radius ratio & $r_{2} / r_{3}$ & 1.13 & \\
Estimated nozzle throat width & $o_{t h}$ & 8.52 & $\mathrm{~mm}$ \\
Inlet blade metal angle & $\beta_{2^{\prime}}$ & -57.50 & $\circ$ \\
Outlet blade metal angle & $\beta_{3^{\prime}}$ & -72.11 & $\circ$ \\
Nozzle inlet blade thickness & $t_{b 2}$ & 1.12 & $\mathrm{~mm}$ \\
Nozzle maximum blade thickness & $t_{\max }$ & 2.70 & $\mathrm{~mm}$ \\
Nozzle outlet blade thickness & $t_{b 3}$ & 0.54 & $\mathrm{~mm}$ \\
\hline \hline
\end{tabular}




\section{Chapter 4}

\section{Computational Turbine Aerodynamic Development}

\subsection{Introduction}

The designed turbine was evaluated with Computational Fluid Dynamics (CFD) to identify areas of improvement and to perform iterative design. The one-dimensional designs of the rotor, nozzles, and volute were implemented in 3D CAD, then modelled in ANSYS CFX 18.1 at the design point. In this chapter, the common CFD setup including solver type, mesh generation, fluid model, and turbulence model are presented. Then, the volute, nozzles, and rotor are investigated with CFD and developed based on the results. Finally, the turbine's on-design results are presented and discussed.

\subsection{Development of Three-Dimensional Components}

This section covers the implementation of the completed one-dimensional design into a preliminary three-dimensional design. The implementation of the rotor was completed in ANSYS BladeGen with further recommendations from literature. Also, the nozzles were implemented in ANSYS BladeGen and the three-dimensional volute was created in SolidWorks without the need for additional information. 


\subsubsection{Rotor Three-Dimensional Implementation}

ANSYS BladeGen was used to create the three-dimensional rotor. The geometry of the inlet and outlet were specified as radial and axial point locations with the information shown in Figure 3.11. Then, to further implement the one-dimensional rotor design in three-dimensions, more information was required regarding the shape of the gas path and blades between the inlet and outlet. Specifically, the hub and shroud meridional profiles and the blade camberline, $\theta^{\prime}$, or blade metal angle, $\beta^{\prime}$, distributions were required. Radial element blades, with no blade lean, were used to limit the produced bending stress and the one-dimensional thickness distribution was implemented directly in BladeGen as shown in Figure 4.1.

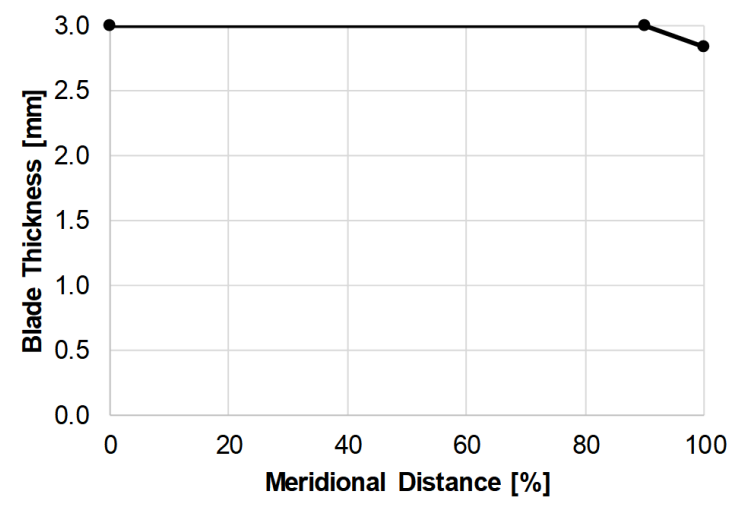

Figure 4.1: Rotor blade thickness distribution.

Rotor blades can be defined in BladeGen based on the $\theta^{\prime}$ or $\beta^{\prime}$ distributions versus the meridional distance or based on the end angle definition. The one-dimensional leading edge and trailing edge metal angles, $\beta_{4^{\prime}}$ and $\beta_{5^{\prime}}$, and a desired trailing edge wrap angle were used to create the $\theta^{\prime}$ and $\beta^{\prime}$ distributions. The hub and shroud metal angles were provided and a Ruled Element spanwise distribution was specified. Bezier control points were used to adjust the metal angles to achieve the desired leading and trailing edge angles and ensure that the throat was located at the trailing edge. The leading edge metal angles at the hub and shroud are equal to the meanline angle of $0^{\circ}$. However, due to the change in radius, the trailing edge metal angles at the hub, $\beta_{h 5^{\prime}}$, and shroud, $\beta_{t 5^{\prime}}$, are calculated based on the meanline metal angle as shown in Equation 4.1 and Equation 4.2. The blade wrap angle specifies the azimuthal location of a specific point on the blade, with respect to the leading edge at $0^{\circ}$ wrap 
angle. For example, different trailing edge wrap angles, $\theta_{t e}^{\prime}$, are shown in Figure 4.2. Based on literature, trailing edge wrap angles usually fall within $40^{\circ}$ to $80^{\circ}$ (Jones, 1994; Sauret, 2012; Jahn, 2015; Fiaschi et al., 2016) so this range was used as the basis for the three-dimensional rotor implementation in BladeGen. Three trailing edge wrap angle, $\theta_{t e}^{\prime}$, cases $\left(40^{\circ}, 60^{\circ}\right.$, and $80^{\circ}$ ), were evaluated in CFD to determine which was best for the present project in terms of design point efficiency as described in Section 4.5.2. The rotor blade metal angle, $\beta^{\prime}$, and wrap angle, $\theta^{\prime}$, distributions for these three examined trailing edge wrap angles are shown in Figure 4.3 .

$$
\begin{aligned}
& \beta_{h 5^{\prime}}=-\tan ^{-1}\left(\frac{r_{5} \tan \left(90-\beta_{5^{\prime}}\right)}{r_{h 5}}\right)-90 \\
& \beta_{t 5^{\prime}}=-\tan ^{-1}\left(\frac{r_{5} \tan \left(90-\beta_{5^{\prime}}\right)}{r_{t 5}}\right)-90
\end{aligned}
$$

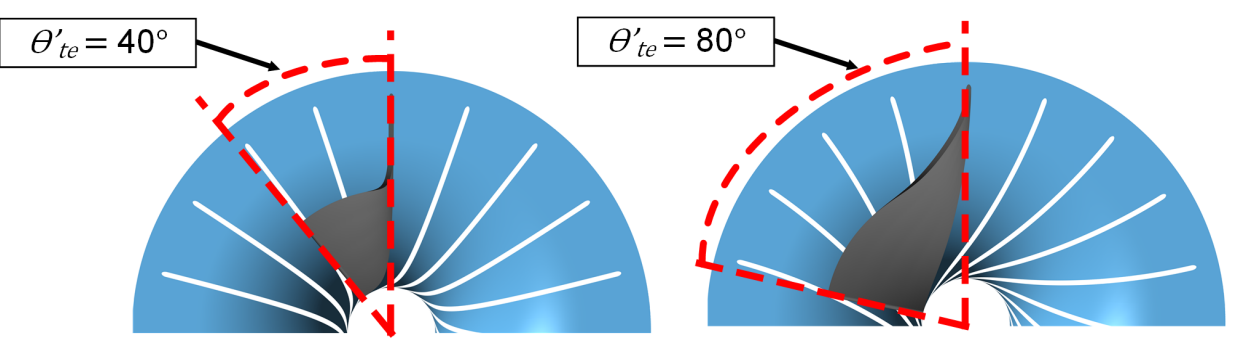

Figure 4.2: Illustration of trailing edge wrap angle.

BladeGen provides a default meridional profile when the program is provided with the rotor inlet and outlet geometry in terms of radial and axial lengths. This default meridional profile is created from Bezier curves defined by the points listed in Table 4.1 and is shown in Figure 4.4a. Aungier (2006) recommends a circular-arc hub contour and a power-law shroud contour for the rotor's meridional profile as summarized in Appendix B. A comparison of the BladeGen default and Aungier meridional profiles is shown in Figure 4.4. It can be seen that the Aungier profile has a reduced cross-sectional area through most of the passage compared to the BladeGen default profile. This reduction is supposed to reduce passage curvature effects such as flow separation from the blades (Aungier, 2006). An abrupt change in the slope of 
the shroud profile at the rotor leading edge is also seen on the Aungier profile. It would be expected that sudden expansions of the flow would not be favourable, but investigating this detail was beyond the scope of the present work. Both profiles have been evaluated in the CFD design stage and the results are discussed in Section 4.5.3.

Table 4.1: Bezier curve control points for default radial turbine meridional profile in BladeGen.

\begin{tabular}{cccc}
\hline \hline \multicolumn{2}{c}{ Hub Profile } & \multicolumn{2}{c}{ Shroud Profile } \\
Axial, $z$ & Radial, $r$ & Axial, $z$ & Radial, $r$ \\
\hline 0 & $r_{4}$ & $b_{4}$ & $r_{4}$ \\
0 & $\left(r_{4}+r_{h 5}\right) / 2$ & $b_{4}$ & $\left(r_{4}+r_{t 5}\right) / 2$ \\
0 & $r_{h 5}$ & $b_{4}$ & $r_{t 5}$ \\
$\left(0+L_{R}\right) / 2$ & $r_{h 5}$ & $\left(b_{4}+L_{R}\right) / 2$ & $r_{t 5}$ \\
$L_{R}$ & $r_{h 5}$ & $L_{R}$ & $r_{t 5}$ \\
\hline \hline
\end{tabular}

Rotor tip clearance was added in BladeGen by removing material from the blade as opposed to expanding the shroud. This is conventional because it maintains the flow area. In BladeGen, a shroud offset layer is created with constant height at the leading and trailing edge and this layer then specifies the tip of the blade.

An example of the rotor in three-dimensions with a $40^{\circ}$ trailing edge wrap angle is shown in Figure 4.5.

\subsubsection{Stator Three-Dimensional Implementation}

ANSYS BladeGen was again used for the three-dimensional implementation of the nozzle row. The nozzle geometry specified in Table 3.4 was specified in BladeGen as radial and axial point locations. The nozzle blade metal angles from the one-dimensional design, $\beta_{2^{\prime}}$ and $\beta_{3^{\prime}}$, were also specified in BladeGen with a linear $\beta^{\prime}$ distribution from the leading edge to trailing edge metal angles and a uniform spanwise distribution was used from hub to shroud. The nozzle metal angle, $\beta^{\prime}$, and camberline wrap angle, $\theta^{\prime}$, distributions are shown in Figure 4.6a. The one-dimensional thickness distribution was implemented directly in BladeGen with a cubic spline profile fit between the leading edge thickness, maximum thickness, and trailing 
edge thickness as shown in Figure 4.6b. The nozzle throat width calculated in BladeGen was $o_{t h}=8.58 \mathrm{~mm}, 0.06 \mathrm{~mm}$ larger than the estimated design value, which was assumed to be acceptable because a larger throat would lead to lower Mach numbers. The throat relates to the nozzle exit flow angle through Equation 3.59, so the resulting flow angles should be monitored in the CFD simulations and adjusted if necessary, as in Section 4.6.

The three-dimensional scroll volute was created by connecting specified cross-sectional geometries at certain azimuthal locations in SolidWorks. Sharp corners were removed from the flow path in the three-dimensional model to avoid simulation troubles by adding fillets. An additional $150 \mathrm{~mm}$ of pipe was added to the volute inlet from the station 1 plane (Figure 2.6) to reduce simulation troubles as well.

Finally, the turbine assembly, with the volute, nozzles, and rotor is shown in Figure 4.7. 


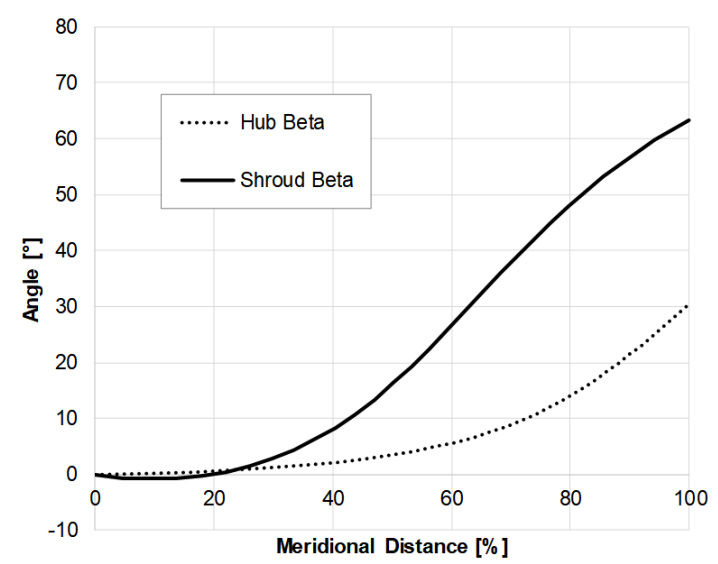

(a) $\beta^{\prime}$ distribution for $\theta_{t e}^{\prime}=40^{\circ}$.

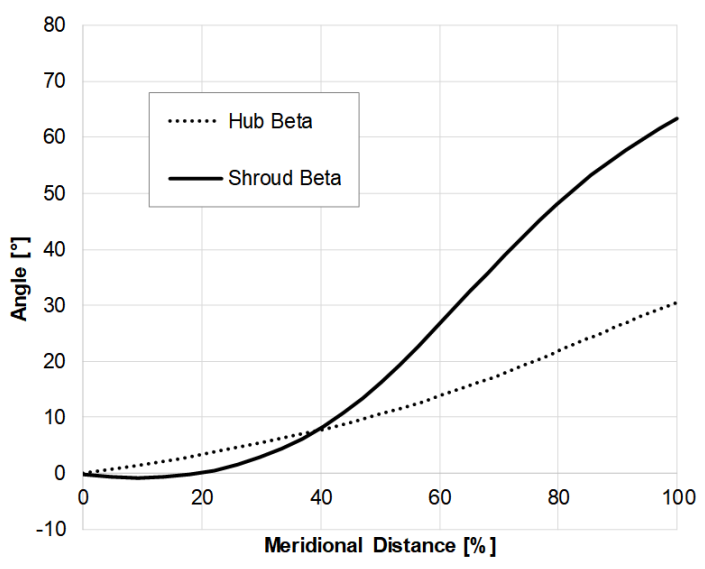

(c) $\beta^{\prime}$ distribution for $\theta_{t e}^{\prime}=60^{\circ}$.

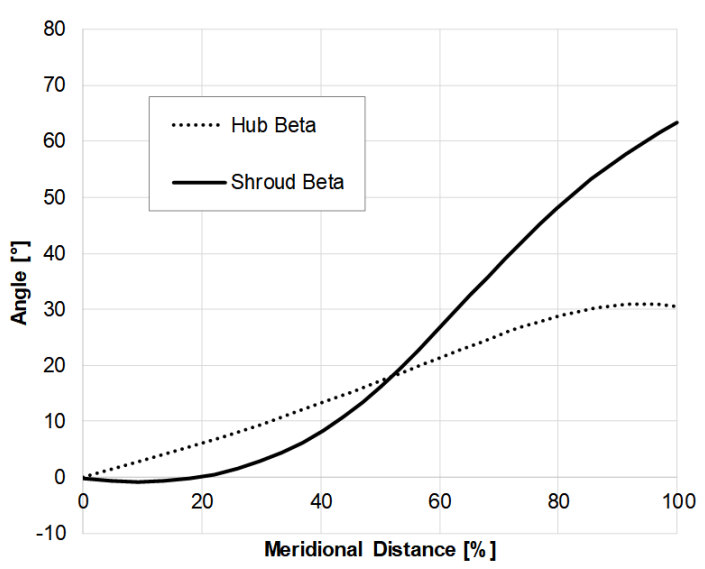

(e) $\beta^{\prime}$ distribution for $\theta_{t e}^{\prime}=80^{\circ}$.

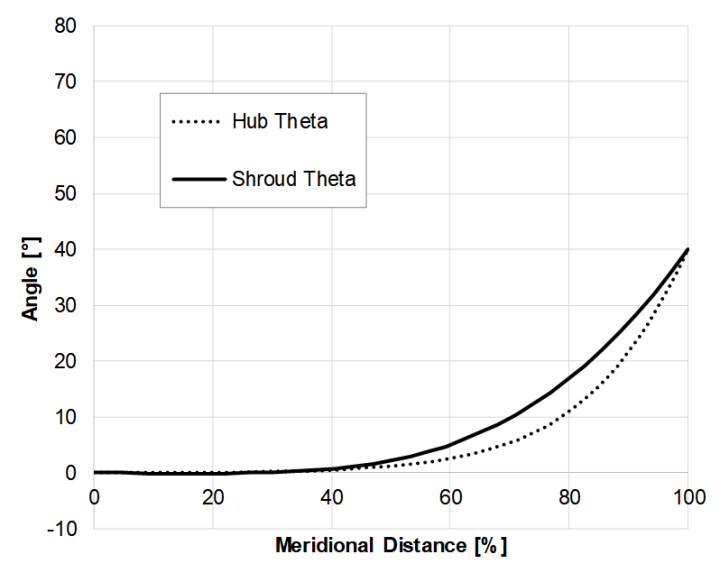

(b) $\theta^{\prime}$ distribution for $\theta_{t e}^{\prime}=40^{\circ}$.

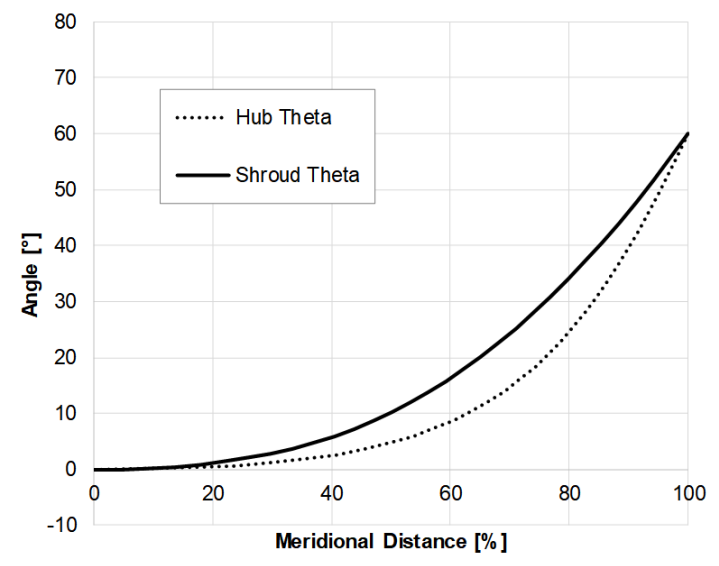

(d) $\theta^{\prime}$ distribution for $\theta_{t e}^{\prime}=60^{\circ}$.

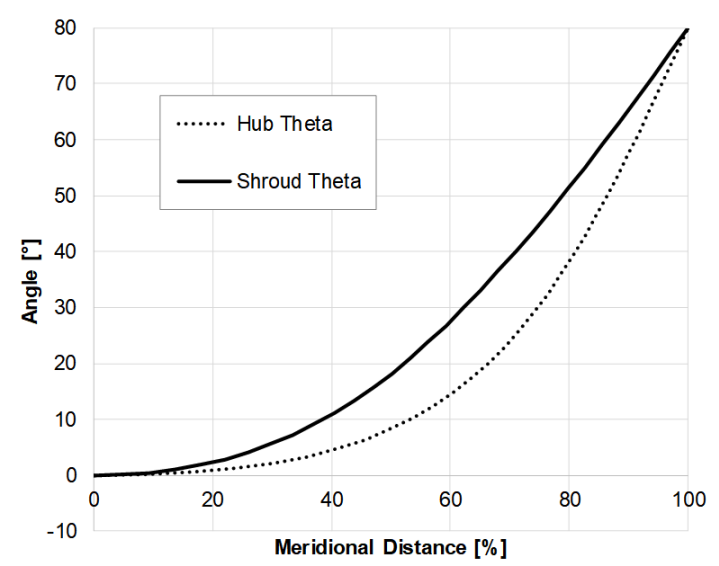

(f) $\theta^{\prime}$ distribution for $\theta_{t e}^{\prime}=80^{\circ}$.

Figure 4.3: Rotor blade metal angle, $\beta^{\prime}$, and wrap angle, $\theta^{\prime}$, distribution for trailing edge wrap angles, $\theta_{t e}^{\prime}$, of $40^{\circ}, 60^{\circ}$, and $80^{\circ}$. 


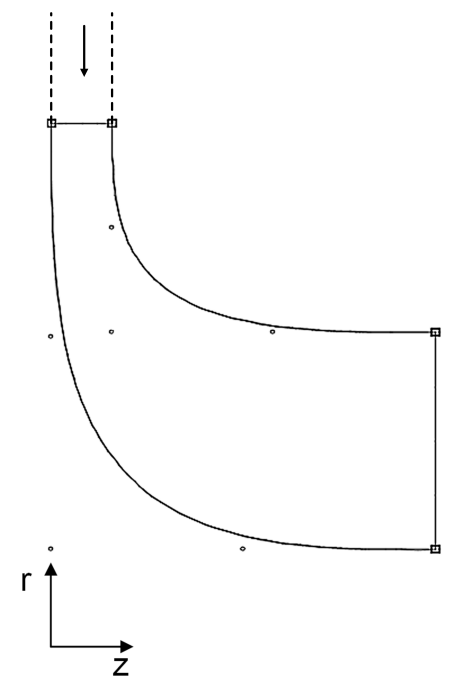

(a) BladeGen profile based on Bezier curves.

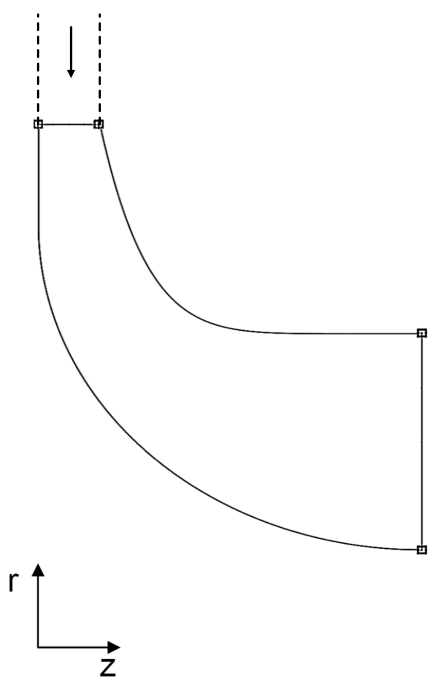

(b) Aungier profile with $n=7$.

Figure 4.4: Comparison of BladeGen default and Aungier meridional profiles.

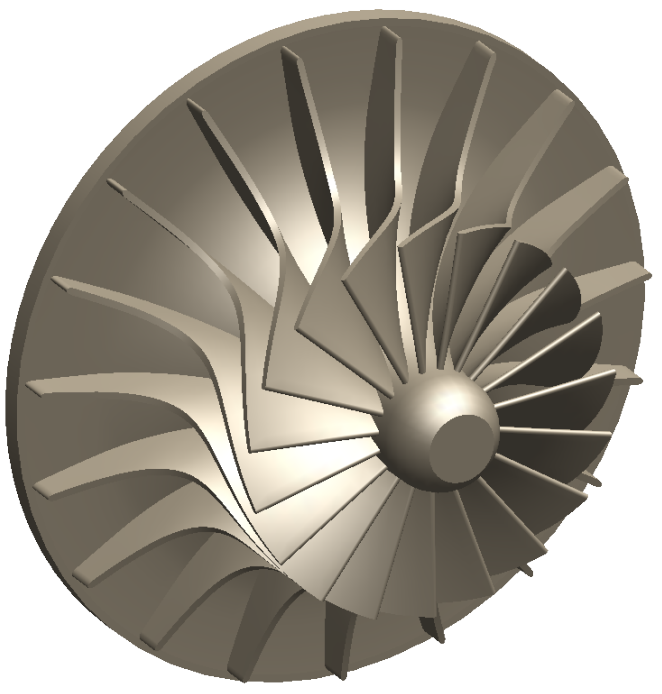

Figure 4.5: Turbine rotor in three-dimensions. 


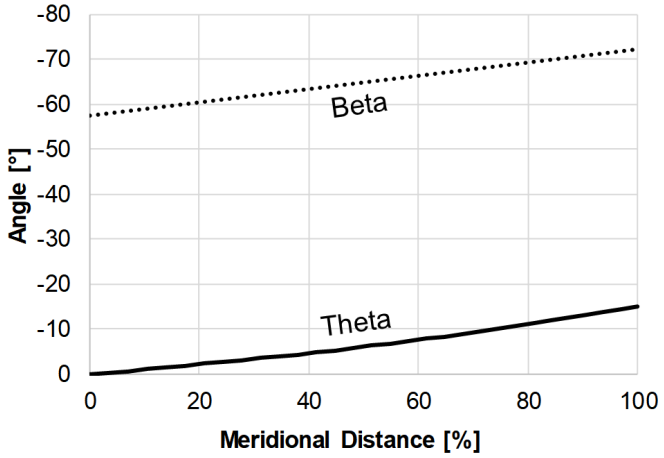

(a) $\beta^{\prime}$ and $\theta^{\prime}$ distribution.

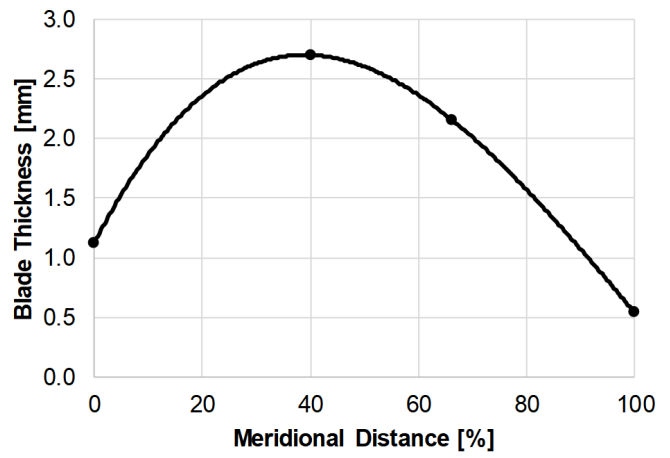

(b) Blade thickness distribution.

Figure 4.6: Nozzle blade angle and thickness distributions.

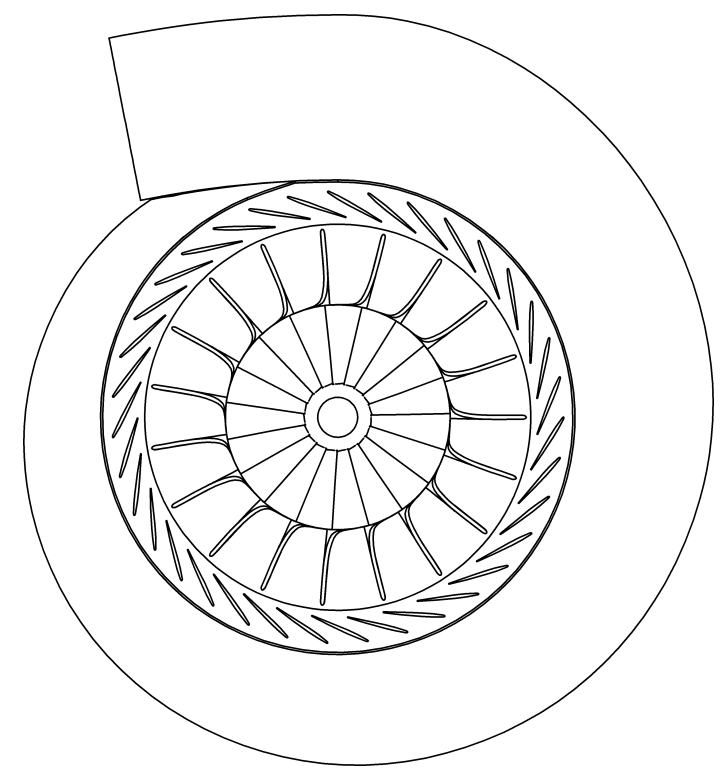

Figure 4.7: Turbine with volute, nozzles, and rotor. 


\subsection{CFD Background}

This section describes the commonalities of the volute, nozzle, and rotor CFD simulations presented in the subsequent sections. The CFD solver, mesh generation, fluid property model, turbulence model, and monitored parameters are discussed. The boundary conditions and further CFD setup are discussed for each simulation set in the following sections.

\section{Solver}

ANSYS CFX 18.1 is the finite volume method CFD solver used for all steady-state, viscous flow CFD simulations in the present work. The Total Energy thermal model was used along with the High Resolution advection scheme in ANSYS CFX to solve the Reynolds-averaged Navier-Stokes equations. The High Resolution scheme combines the robustness of first order accurate schemes and the accuracy of second order accurate schemes by using a blend factor as close to 1 (second order accurate) as much as possible, and lower values (closer to first order accurate) in computationally challenging areas (ANSYS, Inc., 2017b). A timestep around $0.1 / \omega$ was specified for both rotating and stationary frames with some refinement to achieve convergence, as recommended by ANSYS, Inc. (2017a).

\section{Computational Mesh}

ANSYS TurboGrid was used to create the computational mesh for the rotor and nozzle geometries that were created in BladeGen. TurboGrid, with the ATM (Automatic Topology and Meshing) Optimized setting, creates hexahedral meshes suitable for turbomachinery flows. ANSYS Meshing was used to create a tetrahedral mesh for the scroll volute with the Proximity size function and inflation layers specified for all wall boundaries. All meshes included at least 10 inflation layers with an inflation ratio of 1.25 on wall boundaries. The inflation rate was maintained in the rotor tip clearance with at least 20 nodes between the tip and shroud. The first node offset was specified for all wall boundaries of all three components to achieve acceptable $y^{+}$values. 
Initially, meshes with $y^{+}=\sqrt{\tau_{\text {wall }} / \rho}(\Delta y / \nu)$ values around 5 were created to allow use of the SST turbulence model, but this led to computational difficulties due to domain size and mesh quality. The $k-\epsilon$ model was therefore used to allow for higher $y^{+}$values and for robustness in the present work. ANSYS, Inc. (2017b) indicates that the $y^{+}$values required for proper use of the CFX Scalable Wall Functions, which are recommended for the $k-\epsilon$ model, depends on the device Reynolds number. The machine Reynolds number (Equation 2.23) for the rotor, based on the rotor radius and rotor inlet conditions was $6.9 \times 10^{7}$. According to Sauret \& Gu (2014), based on this machine Reynolds number and the chosen turbulence model, the maximum $y^{+}$values should be below 500. Future work should verify whether this limit is indeed applicable for the present work as well. Nevertheless, a $y^{+}$independence test was performed for the rotor to find the value of $y^{+}$where the CFD simulations became independent and the results are shown in Figure 4.8a. Acceptable accuracy and good convergence was achieved for the rotor with meshes with maximum $y^{+}$values less than 235 . This maximum $y^{+}$ value was assumed to be adequate for the nozzle and volute meshes as well. Mesh independence studies were performed to ensure that the simulations were independent of the mesh in terms of the total number of nodes. The results of these studies are presented in Figure $4.8 \mathrm{~b}$ for the rotor, Figure 4.9 for the nozzles, and Figure 4.10 for the volute, where the number of nodes is the value for a single blade passage for the nozzles and rotor. Statistics of the $y^{+}$values at each wall location for each component are shown in Table 4.2. It can be seen that each location has average $y^{+}$values below 150 and standard deviations below 60 indicating that their maximum values occur only in a small region. The final single passage rotor and nozzle meshes had 2.3 million and 0.5 million nodes, and the final volute mesh had 1.6 million nodes. The details of the meshes used are shown in figures included in Appendix C.

\section{Fluid Model}

The properties of S-CO2 were implemented in ANSYS CFX with RGP (real gas property) tables created with data from REFPROP. ANSYS CFX uses these tables by interpolating between the properties at pressure and temperature state points and ANSYS, Inc. (2017a) details the use and features of RGP tables in more depth. Each RGP file contains a total of 


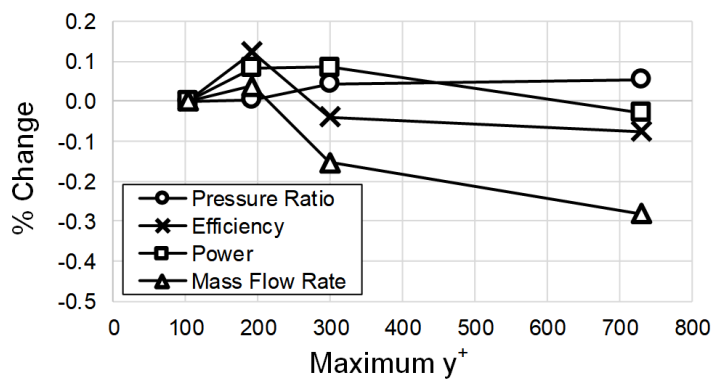

(a) $y^{+}$independence study for rotor.

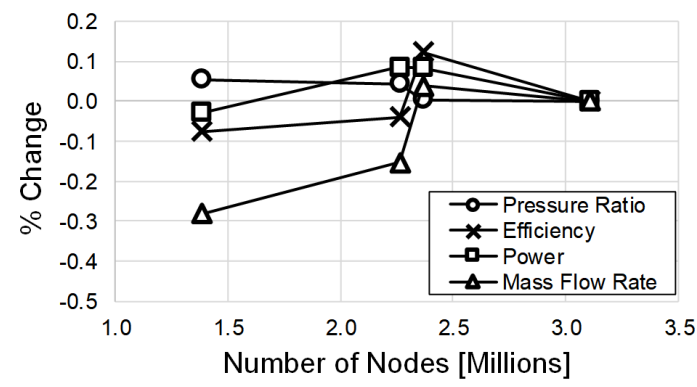

(b) Node independence study for rotor.

Figure 4.8: Mesh independence studies for rotor.

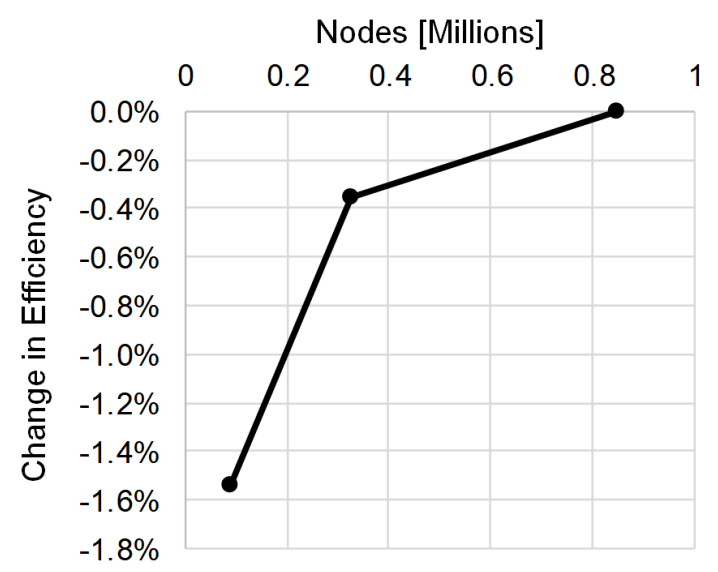

Figure 4.9: Mesh independence study for nozzles.

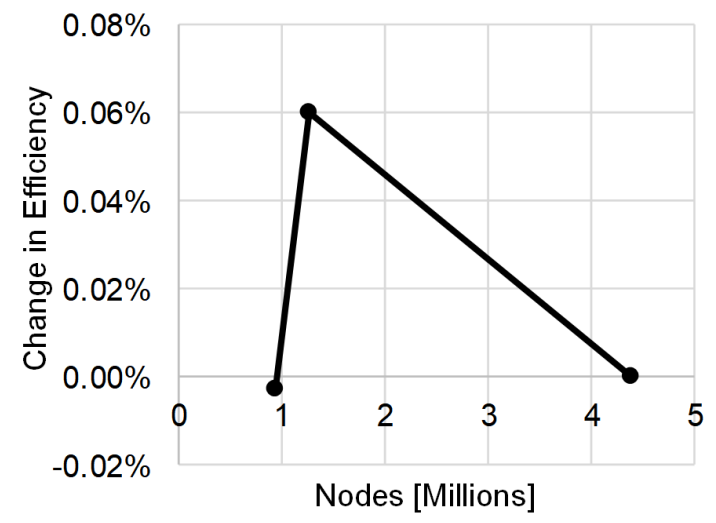

Figure 4.10: Mesh independence study for volute.

nine property tables to define a fluid. The nine tables list the specific enthalpy, speed of sound, specific volume, specific heat at constant volume, specific heat at constant pressure, partial derivative of pressure with respect to specific volume at constant temperature, specific entropy, 
Table 4.2: Volute, nozzle, and rotor wall $y^{+}$values.

\begin{tabular}{l|cccc|ccc|c}
\hline \hline & \multicolumn{4}{|c|}{ Rotor } & \multicolumn{4}{c|}{ Nozzle } \\
& Hub & Shroud & Blade Walls & Blade Tip & Hub & Shroud & Blade Walls & Walls \\
\hline Average & 91 & 128 & 91 & 143 & 50 & 50 & 49 & 133 \\
Maximum & 204 & 202 & 230 & 213 & 88 & 78 & 92 & 235 \\
Std. Dev. & 42 & 35 & 35 & 29 & 15 & 14 & 15 & 56 \\
\hline \hline
\end{tabular}

dynamic viscosity, and thermal conductivity. Because the property values are interpolated from the tables within ANSYS CFX, the grid spacing for the tabulated independent variables, pressure and temperature, is important. A script provided by Robin Steed of ANSYS Inc. presented in Appendix D has been used to create the RGP tables for the present work. The script calls functions within the ANSYS software package which build the tables with data from REFPROP. The required spacing is determined iteratively by creating an initial table and then interpolating for values between each grid point. If the error between the interpolated value and the REFPROP value is higher than a specified acceptable error, then the local spacing is refined at that point and the process is repeated until the acceptable error is achieved for each grid point. The final RGP tables used for the present work are also presented in Appendix D.

\section{Turbulence Model}

The $k-\epsilon$ model was used due to computational mesh limitations and for robustness in the present work with first order accuracy using the upwind advection scheme for robustness. First order accuracy was used for solving the turbulence model only, whereas the High Resolution scheme was used for the solving the Reynolds-averaged Navier-Stokes equations. Scalable wall functions were used to estimate the flow's boundary layer in terms of wall shear stress. The laminar-turbulent transition for a flat plate is generally accepted to occur at the critical Reynolds number of $5 \times 10^{5}$ (Cengel, 2007). The flat plate equivalent Reynolds number (Equation 4.3) based on the rotor blade meridional path length and the turbine inlet conditions indicates that this critical Reynolds number is reached at a meridional distance of $2.2 \%$ of the total blade length as shown in Figure 4.11. Based on this, the flow was assumed to be 
fully turbulent for the purposes of the present work and the $k-\epsilon$ model without a transition model was assumed to be acceptable.

$$
R e_{F P}=\frac{\rho_{4} W_{4} L_{m}}{\mu_{4}}
$$

where $L_{m}$ is the rotor blade meridional path length at the hub.

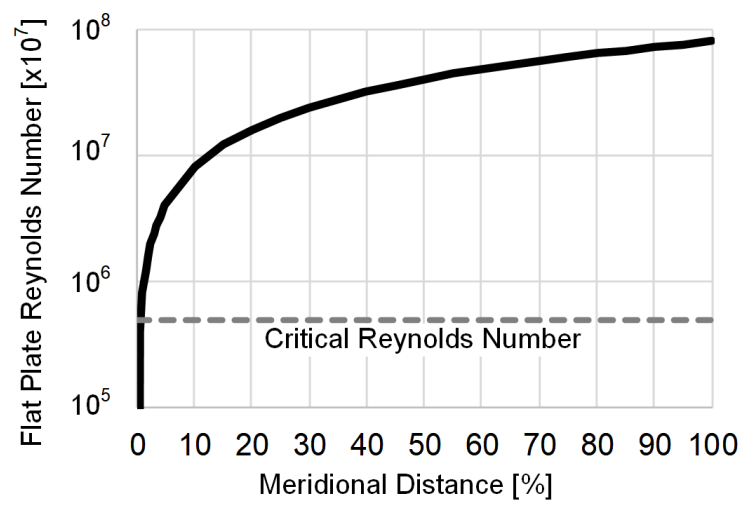

Figure 4.11: Rotor flat plate Reynolds number versus meridional distance to locate laminarturbulent transition.

\section{Monitored Parameters}

Key output variables such as the inlet and outlet pressure and temperature, mass flow, pressure ratio, efficiency, and output power were monitored along with the root mean square residuals for each CFD simulation to determine convergence. A simulation was assumed to be converged when the root mean square residuals were below $10^{-6}$ and/or the mass flow error was less than $0.5 \%$, and both the standard deviations of the pressure ratio and output power were less than $0.01 \%$ of their average for the previous 20 iterations.

\subsection{Computational Volute Development}

The volute was evaluated in ANSYS CFX at the design point with steady-state simulations to evaluate the performance. Total pressure and temperature, $P_{01}$ and $T_{01}$, were specified at the volute inlet with a uniform velocity profile, neglecting the boundary layer that would be present 
due to the upstream delivery pipe. A $28.8 \mathrm{~mm}$ radial extension was added beyond the volute outlet, $r_{2 \mathrm{v}}$, as shown in Figure 4.12 to allow for the flow to mix out before the volute outlet boundary where the mass flow rate was specified with the pressure shape constrained based on a Pressure Profile Blend of 0.9. Finally, the wall boundaries were specified as adiabatic, smooth, no-slip walls.

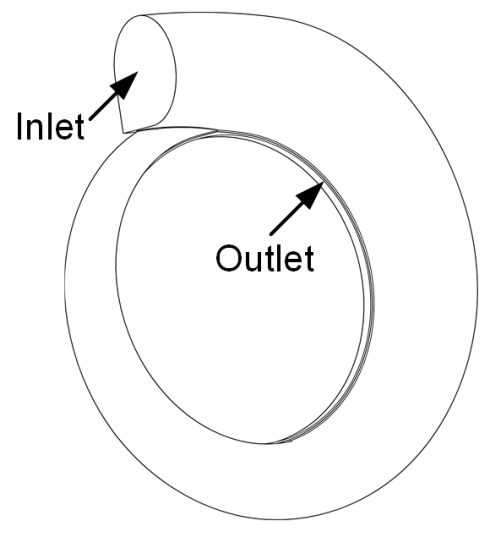

(a) No extension.

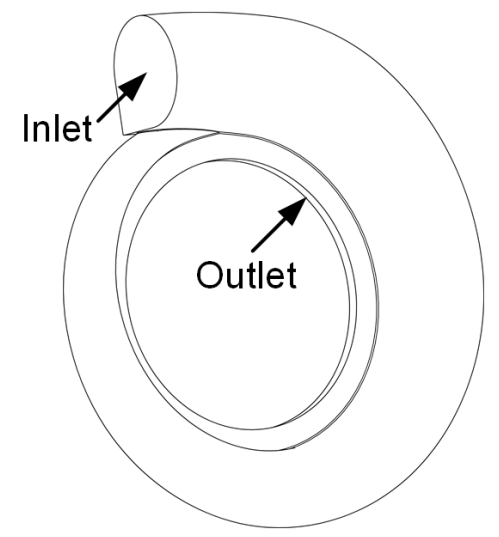

(b) $28 \mathrm{~mm}$ radial extension.

Figure 4.12: Volute domain without and with radial extension at outlet.

Initially, the turbulence conditions at the volute inlet were not known, so the inlet turbulence was varied until the gradient at the inlet was reduced because a large gradient was not expected. First, a default value was specified in the initial simulations. In CFX, the Medium Intensity option, which defines a $5 \%$ turbulence intensity and an eddy viscosity ratio of 10 , was used. The eddy dissipation through the volute in this case is shown in Figure 4.13 and a similar distribution was seen for the turbulence kinetic energy.

The volute inlet turbulence boundary conditions were adjusted such that a reduced gradient of turbulent kinetic energy, $k$ and eddy dissipation, $\epsilon$, was seen at the inlet. The inlet turbulence was not found to have a significant effect on the simulation as shown in Table 4.3. However, the turbulence gradient at the inlet was reduced with the specification of a kinetic energy and eddy dissipation of $0.2 \mathrm{~m}^{2} / \mathrm{s}^{2}$ and $500 \mathrm{~m}^{2} / \mathrm{s}^{3}$ at the inlet as shown in Figure 4.14, so this setup was used.

The performance of the volute can be evaluated in terms of the uniformity of the flow around the periphery of the volute outlet and the velocity magnitude and angle of the flow 


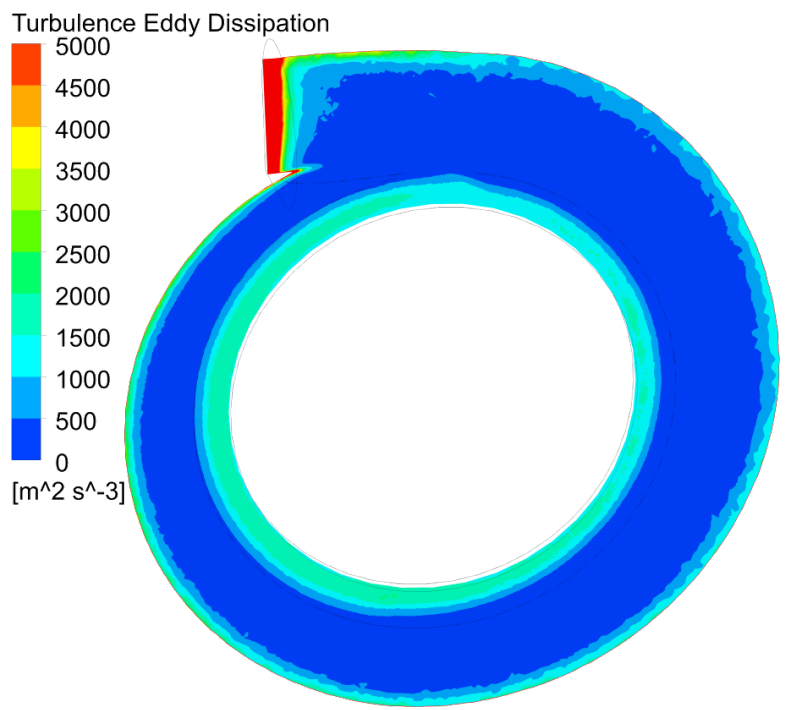

Figure 4.13: Turbulence eddy dissipation through a representative cross-section of the volute $(z=7.1 \mathrm{~mm})$ for the case where Medium Intensity is specified at the volute inlet.

Table 4.3: Percent change of volute pressure loss coefficient for different volute inlet turbulence cases.

\begin{tabular}{lc}
\hline \hline Inlet Turbulence & \\
Boundary Condition & $K_{v}$ \\
\hline Medium Intensity & $0.17 \%$ \\
$k=1 \mathrm{~m}^{2} / \mathrm{s}^{2}, \epsilon=5000 \mathrm{~m}^{2} / \mathrm{s}^{3}$ & $0.11 \%$ \\
$k=0.2 \mathrm{~m}^{2} / \mathrm{s}^{2}, \epsilon=500 \mathrm{~m}^{2} / \mathrm{s}^{3}$ (Baseline) & - \\
\hline \hline
\end{tabular}

delivered to the inlet of the nozzle blades at $r_{2}$. Japikse \& Baines (1994) discuss the effect that mixing and recirculation of the flow at the volute tongue location has on the uniformity of the flow at the volute outlet. They present the plots shown in Figure 4.15 to illustrate how much variation may be expected for well-designed volutes.

The one-dimensional volute design was found to achieve similar uniformity as presented by Japikse \& Baines (1994) with a variation of less than $20 \%$ for the normalized velocity and less than $10^{\circ}$ for the flow angle as shown in Figure 4.16. However, the average flow angle at the nozzle inlet radius, $r_{2}$, was $63^{\circ}$ whereas the one-dimensional nozzle design required $57^{\circ}$. It was found that increasing the volute Swirl Coefficient, $S_{c}$, reduced the swirl or tangential component of velocity and thus the flow angle. The effect of varying the Swirl Coefficient on the 


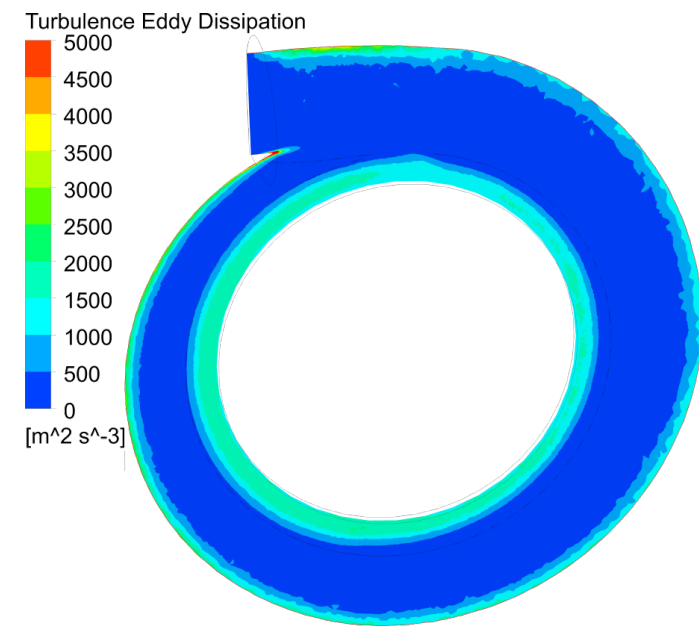

(a) Coarse scale, $\epsilon=0-5000 \mathrm{~m}^{2} / \mathrm{s}^{3}$.

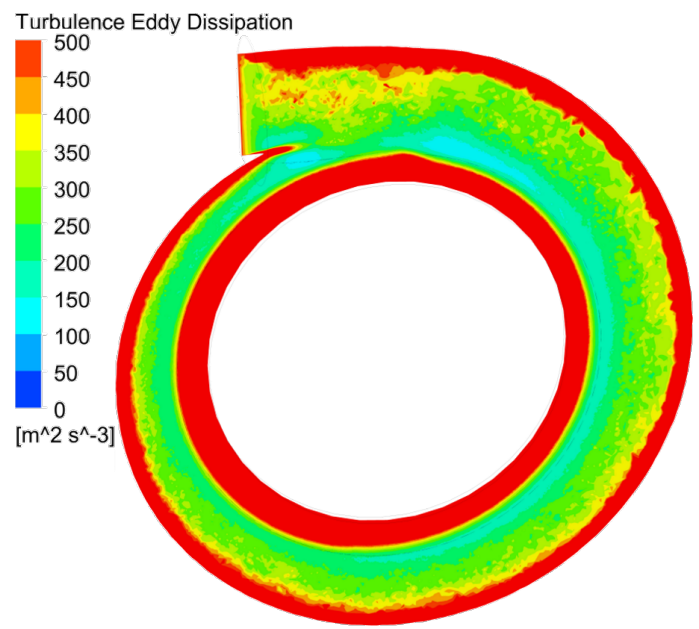

(b) Fine scale, $\epsilon=0-500 \mathrm{~m}^{2} / \mathrm{s}^{3}$.

Figure 4.14: Turbulence eddy dissipation through a representative cross-section of the volute $(z=7.1 \mathrm{~mm})$ for the case where $k=0.2 \mathrm{~m}^{2} / \mathrm{s}^{2}$ and $\epsilon=500 \mathrm{~m}^{2} / \mathrm{s}^{3}$ are specified at the volute inlet.
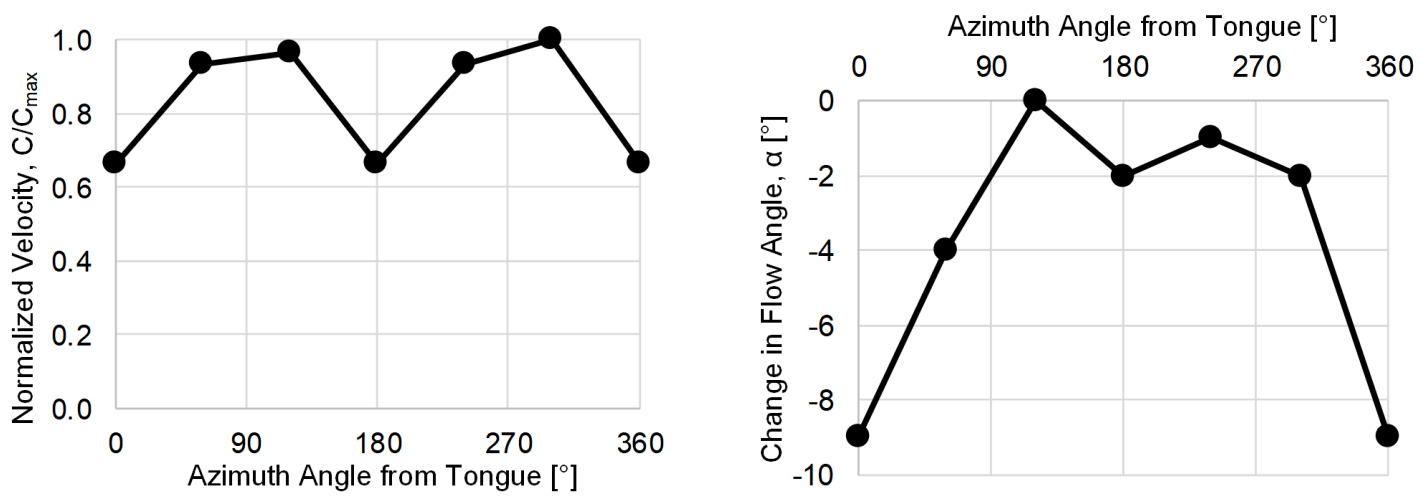

Figure 4.15: Example variation of flow around periphery of volute outlet (Adapted from Japikse \& Baines, 1994).

flow angle at $r_{2}$ is shown in Table 4.4, where the range of $S_{c}$ is based on the recommendation from Japikse \& Baines (1994). A value of $S_{c}=0.95$ was used for the final volute design, because it provided $\alpha_{2 a v g}$ closest to the design value of $57^{\circ}$ and a more uniform flow around the periphery of the volute outlet, as shown in Figure 4.17. 

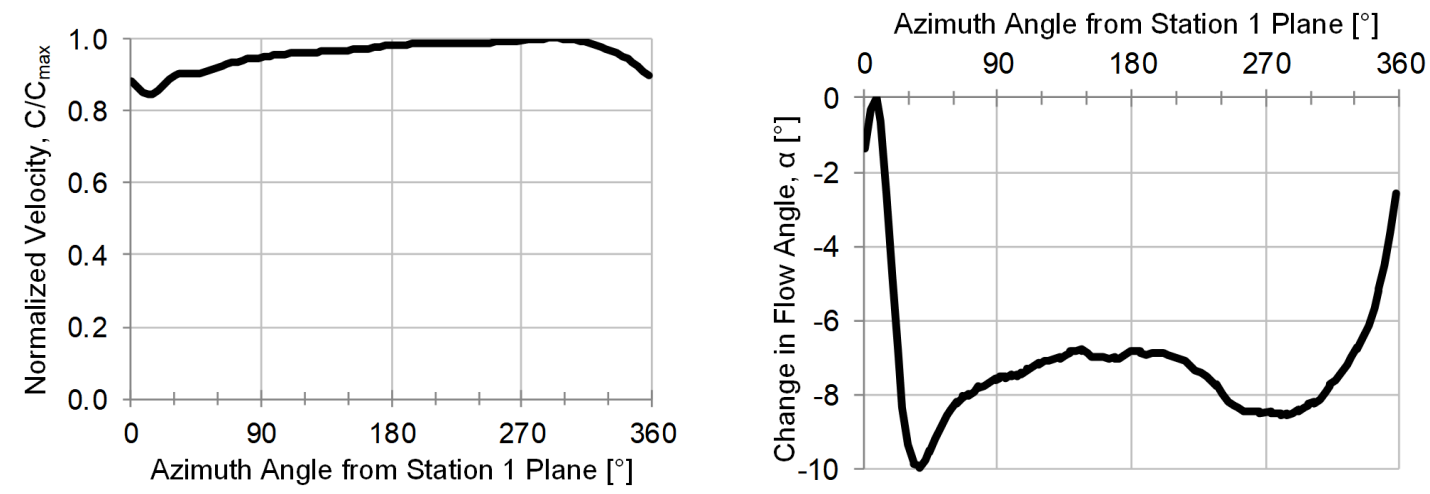

Figure 4.16: Variation of flow around periphery of volute outlet with $S_{c}=0.85$. (Tongue location is $40^{\circ} \mathrm{CCW}$ from the station 1 plane.)

Table 4.4: Effect of volute Swirl Coefficient on nozzle inlet flow angle.

\begin{tabular}{lr}
\hline \hline$S_{c}$ & $\alpha_{2 a v g}$ \\
\hline 0.85 & $63^{\circ}$ \\
0.90 & $61^{\circ}$ \\
0.95 & $59^{\circ}$ \\
\hline \hline
\end{tabular}
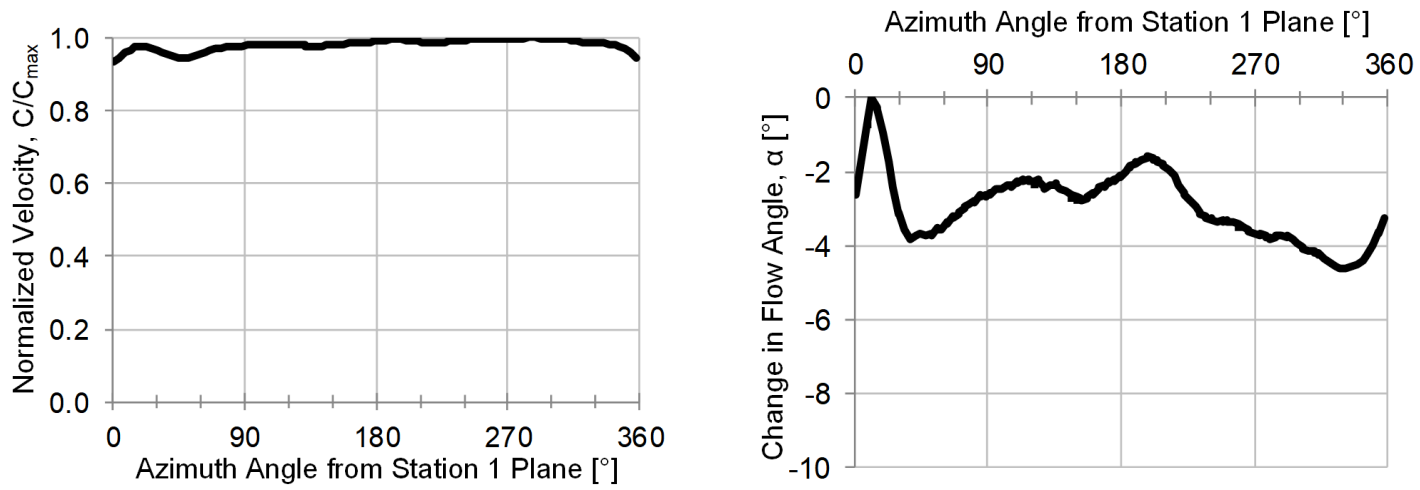

Figure 4.17: Variation of flow around periphery of volute outlet with $S_{c}=0.95$. (Tongue location is $40^{\circ} \mathrm{CCW}$ from the station 1 plane.)

\subsection{Computational Rotor Development}

The rotor was evaluated in $\mathrm{CFD}$ at the design point with steady-state simulations to evaluate the performance and identify areas of improvement. A single rotor blade passage was simulated, taking advantage of the rotational periodicity and applying periodic boundary 
conditions. The rotor rotational speed was specified such that the passage was in the rotating frame at the design $R P M$. The CFD simulations were setup with the total conditions, $P_{04}$ and $T_{04}$, and the absolute flow angle, $\alpha_{4}$, specified at the rotor inlet boundary and the mass flow rate specified at the rotor outlet boundary with the pressure shape constrained based on a Pressure Profile Blend of 0.9. The wall boundaries were specified as adiabatic, smooth, no-slip walls. The turbulence conditions at the rotor inlet boundary were specified based on conditions at the rotor inlet boundary radius from the volute simulations of the previous section as $k=20 \mathrm{~m}^{2} / \mathrm{s}^{2}$ and $\epsilon=5000 \mathrm{~m}^{2} / \mathrm{s}^{3}$. The inlet boundary for these simulations was specified upstream of the rotor leading edge by $8 \%$ of the total rotor blade meridional length to a radius of $155 \mathrm{~mm}$. The outlet boundary was extended downstream of the rotor trailing edge with a constant geometry annular passage by $30 \%$ of the total rotor blade meridional length to an axial length of $140 \mathrm{~mm}$, where the rotor wakes were seen to be mixed out. These simulations were limited in that the inlet flow was uniform, neglecting the boundary layer and wakes that would be present after the flow leaves the nozzles. However, it was assumed that the evaluation of the meanline flow in these simulations could provide insights reflective of those that would be seen with conditions based on the upstream components.

In the next sections, the effect of tip clearance is investigated, the effect of trailing edge wrap angle is investigated, and the hub and shroud meridional profile options are compared.

\subsubsection{Tip Clearance}

The amount of clearance between the blade tip and the shroud casing can have a significant impact on efficiency as shown by Futral \& Holeski (1970). The smallest possible clearance is desired for maximum performance, but machining tolerances and the expansion of the rotor require a certain value of tip clearance. Qi et al. (2017) limited their S-CO2 radial turbine design to a minimum clearance of $0.1 \mathrm{~mm}$ or $10 \%$ of their blade height based on machining tolerances and the uncertainty of thermal expansion. Similarly, Sauret \& Gu (2014) and White (2015) used tip clearances of $0.3 \mathrm{~mm}$ and $0.4 \mathrm{~mm}$ for their Organic Rankine Cycle radial turbines. This range guided the investigation of tip clearance effects for the present S-CO2 turbine. 
Equal axial and radial clearances were specified at the rotor inlet and outlet. The effect of the tip clearance gap on the rotor efficiency is shown in Figure 4.18. The trend is generally linear which is in good agreement with the results of Futral \& Holeski (1970). A uniform tip clearance of $0.3 \mathrm{~mm}$ in both the axial and radial direction was selected for the present work based on the values seen in similar works such as Sauret \& Gu (2014) and White (2015). The rotor inlet blade height was $14.2 \mathrm{~mm}$, so the selected tip clearance of $0.3 \mathrm{~mm}$ would be $2.1 \%$ of the span which falls within the range of interest quoted by Futral \& Holeski (1970).

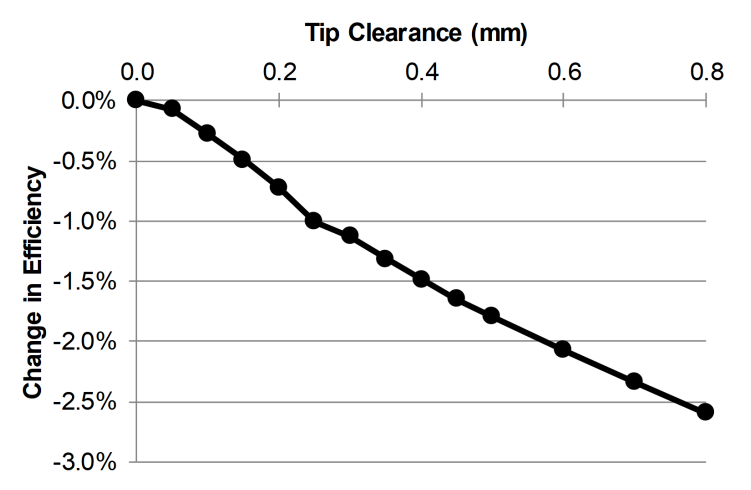

Figure 4.18: Change in rotor efficiency versus tip clearance gap.

\subsubsection{Wrap Angle}

The rotor leading edge and trailing edge blade metal angles can be input directly into BladeGen, but a trailing edge wrap angle, $\theta_{t e}^{\prime}$, is required to define the blade camber angle and complete the blade angle definition. For this reason, a parametric study of the effect of trailing edge wrap angle on the rotor performance was performed. From Figure 4.2, it can be seen that higher $\theta_{t e}^{\prime}$ values define longer blades.

Trailing edge wrap angles of $40^{\circ}, 50^{\circ}, 60^{\circ}$, and $80^{\circ}$ were evaluated in CFD. Values below $40^{\circ}$ produced a large gradient in the metal angle distribution which Aungier (2006) indicates is unfavourable for these machines because the flow has trouble remaining attached to the blade, so $40^{\circ}$ was used as the minimum. The effect of $\theta_{t e}^{\prime}$ on the rotor efficiency is shown in Figure 4.19 where a generally linear decrease in efficiency is seen as the wrap angle is increased from $40^{\circ}$ to $80^{\circ}$. This drop in efficiency may be attributed to more profile or frictional losses 
as the flow path length and blade wetted surface area are increased. Also, it is hypothesized that the increase in the tip clearance streamwise length due to the increase in blade length means that more fluid is passed through the gap generating more secondary flows and more losses. Detailed analysis of the change in flow mechanics due to the change in $\theta_{t e}^{\prime}$ was beyond the scope of the present work, so the $\theta_{t e}^{\prime}=40^{\circ}$ was selected based on the peak efficiency.

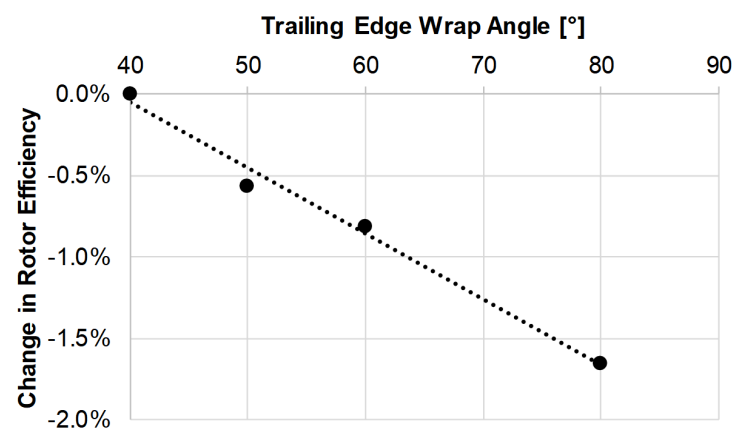

Figure 4.19: Change in rotor efficiency versus trailing edge wrap angle.

\subsubsection{Hub and Shroud Meridional Profile}

The hub and shroud meridional profiles were investigated by comparing the BladeGen default meridional and Aungier recommended meridional profiles in CFD. Aungier (2006) indicates that their profile method expands the flow with a more gradual increase in flow area in an attempt to reduce losses. The two profiles have been previously presented in Figure 4.4.

The Aungier meridional profile provides higher efficiency than the BladeGen default as indicated by Table 4.5. The effect of the different profiles can be visualized in terms of the Mach number on the meridional plane as shown in Figure 4.20. The Aungier profile is seen to have less of a low speed area at both the turn of the hub and the turn of the shroud, and a more gradual change in Mach number from leading edge to trailing edge. The Aungier profile was selected because it provided the higher efficiency. The Aungier profile is not perfect as the low Mach number region at the hub still exists and the abrupt change in shroud profile slope at the rotor leading edge may not be favourable, but optimizing the meridional path was beyond the scope of the present work. 
Table 4.5: Rotor efficiency for BladeGen default and Aungier meridional profiles.

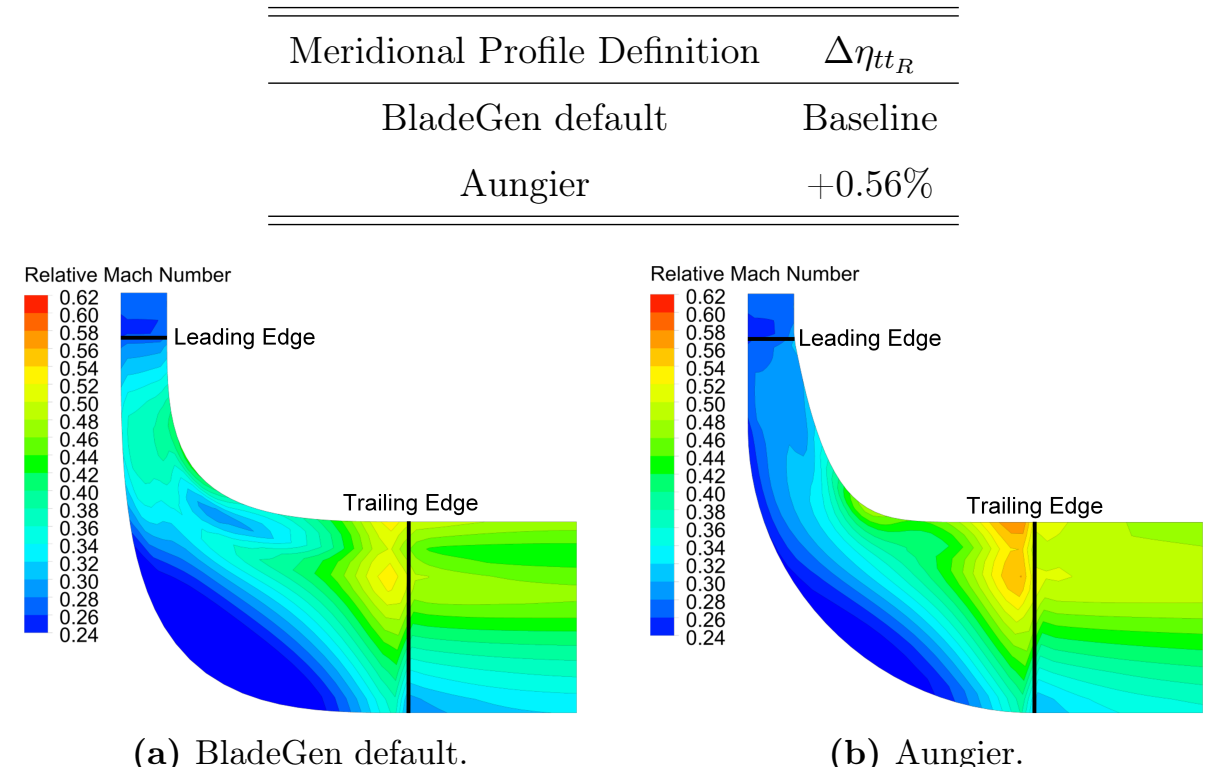

(a) BladeGen default.

(b) Aungier.

Figure 4.20: Comparison of Mach number distribution on meridional plane for BladeGen default and Aungier meridional profiles.

\subsection{Computational Nozzle and Rotor Development}

The nozzle and rotor were evaluated in CFD in tandem at the design point with steadystate simulations to evaluate the performance and identify areas of improvement. A single rotor passage with a pitch of $18.95^{\circ}$ and a single nozzle passage with a pitch of $10.3^{\circ}$ were simulated together, utilizing the rotational periodicity and periodic boundary conditions. A stage (mixing plane) interface with constant total pressure was used for the nozzles-rotor interface at a radius of $145 \mathrm{~mm}$. The rotor rotational speed was specified such that the passage was in the rotating frame at the design $R P M$, and the nozzles were stationary. The nozzle inlet conditions were specified by profile data obtained from the volute simulations at the nozzle inlet radius. The azimuthal location of the profile data used for the nozzle inlet was $70.9^{\circ}$ to $90^{\circ}$ from the station 1 plane which was selected as a location assumed to be representative of the whole azimuth based on Figure 4.17. The total pressure and temperature, $P_{02}$ and $T_{02}$, were specified at the nozzle inlet along with unit vector velocity components in the radial, tangential, and axial direction. The mass flow rate was specified at the rotor outlet with the 
pressure shape constrained based on a Pressure Profile Blend of 0.9 and the wall boundaries were specified as adiabatic, smooth, no-slip walls, like was done for the simulations of the rotor only. The CFD results presented are boundary average properties with the boundaries being offset from the nozzle and rotor blades to avoid their effects according to Table 4.6.

Table 4.6: Property evaluation locations offset from blade leading and trailing edges.

\begin{tabular}{lccccc}
\hline \hline Location & \multicolumn{2}{c}{ Physical Location } & \multicolumn{2}{c}{ CFD Evaluation Location } \\
& Axial, $z$ & Radial, $r$ & Axial, $z$ & Radial, $r$ \\
\hline Nozzle Trailing Edge & 0 to $b_{4}$ & $r_{3}$ & 0 to $b_{4}$ & $0.99 r_{3}$ \\
Rotor Leading Edge & 0 to $b_{4}$ & $r_{4}$ & 0 to $b_{4}$ & $1.01 r_{4}$ \\
Rotor Trailing Edge & $L_{R}$ & $r_{h 5}$ to $r_{t 5}$ & $1.05 L_{R}$ & $r_{h 5}$ to $r_{t 5}$ \\
\hline \hline
\end{tabular}

\subsubsection{Nozzle Metal Angle Adjustment}

The nozzle leading and trailing edge metal angles were adjusted to be compatible with the flow provided by the volute. This involved checking the incidence of the flow at the leading edge and the relative flow angle delivered to the rotor inlet. The nozzle metal angles at the leading and trailing edge were adjusted according to these checks.

The optimum nozzle inlet incidence, $i^{*}$, from the one-dimensional design was $0.57^{\circ}$. The average incidence in the CFD results, based on nozzle inlet conditions from the volute simulations and the one-dimensional design nozzle metal angles from Table 3.4, was 0.67 ${ }^{\circ}$. Figure 4.17 indicates that the nozzle inlet flow angle may vary by up to $2.5^{\circ}$ from the average, but the effect of that variation on the nozzle performance was not investigated in the present work. Figure 4.21 shows the nozzle blade loading in terms of the local static pressure normalized by the nozzle inlet total pressure for a simulation corresponding to the average incidence value of $0.67^{\circ}$. The incidence results in a dip on the blade loading chart just after the leading edge where the flow is not fully attached to the blade. This separated region is only seen to affect about $5 \%$ of the flow on the suction side according to the blade loading chart, then the flow attaches to the blade and is able to follow the profile. This separated flow at the leading edge and the attachment of the flow soon after is confirmed in Figure 4.22 where the Mach 
number is seen to increase gradually through the passage. The effect of the incidence here was not seen to have a noticeable effect on the nozzle performance because the total pressure loss coefficient of $K_{N}=0.04$ was still low according to Japikse \& Baines (1994), so the incidence was not adjusted in the present work. However, adjustment of the leading edge metal angle and the incidence angle should be investigated in the future because the effect of the incidence may be more pronounced in off-design operation. White (2015) mentioned that increasing the leading edge thickness would help the flow stay attached at the leading edge for a wider range of incidence angles, so this may be something to investigate as future work.

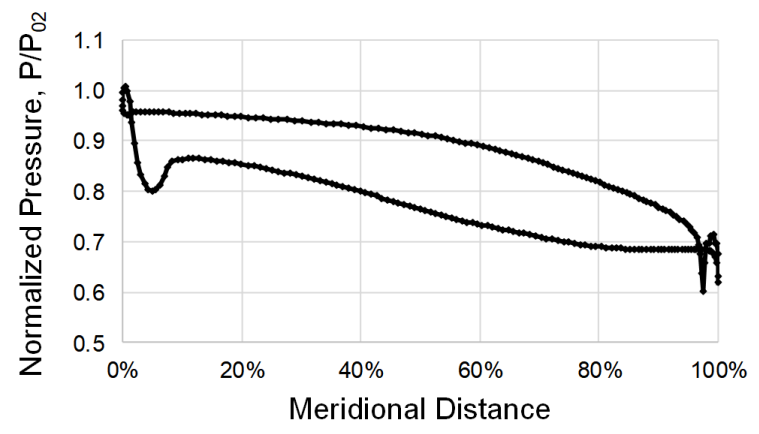

Figure 4.21: Nozzle blade loading at $50 \%$ span.

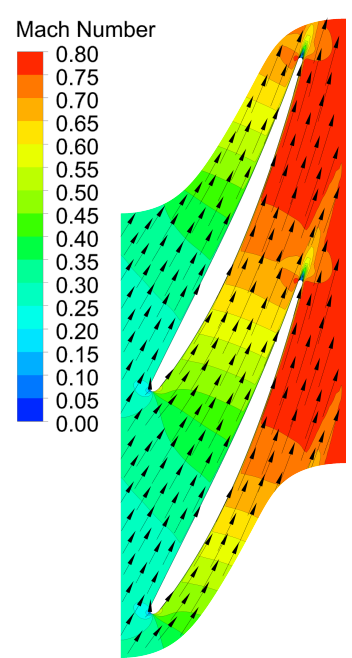

Figure 4.22: Mach number and velocity vectors through nozzle row at 50\% span.

The rotor inlet velocity triangle resulting from the one-dimensional design metal angles $\left(\beta_{3^{\prime}}=72.11^{\circ}\right)$ is shown in Figure 4.23 in comparison to the designed velocity triangle. The 
velocities and flow properties were evaluated at a radius of $142.9 \mathrm{~mm}, 1.3 \mathrm{~mm}$ before the rotor leading edge where the flow was found to be more uniform and less affected by the distortion of the flow due to the blades. The design values were shifted from the rotor leading edge or inlet radius to this CFD evaluation location with the methods presented as Equation 3.48 through Equation 3.53. For the comparison of the velocity triangles, the design values are the meanline values from the preliminary design whereas the velocities obtained from the CFD results are the area-averaged values over the whole boundary at the CFD evaluation location. The difference between the design case and the CFD case may be attributed to the difference in flow conditions, the averaging of the fluid properties across the stage mixing plane, and the flow deviation at the nozzle trailing edge that was not accounted for in the one-dimensional design. The total flow properties at the rotor inlet were less than the design case because of losses through the volute and nozzle row. The flow properties from the CFD results at the rotor inlet with $\beta_{3^{\prime}}=-72.11^{\circ}$ are compared to the design conditions in Table 4.7.

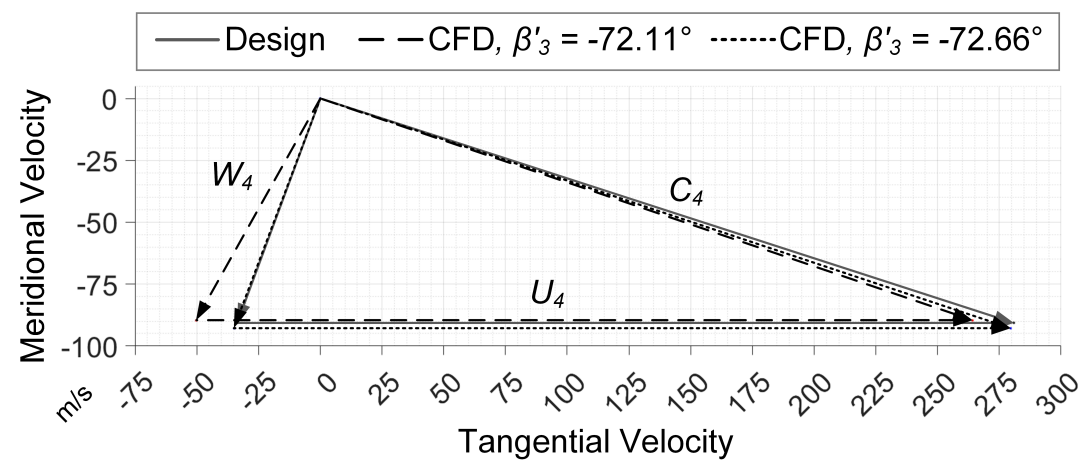

Figure 4.23: Comparison of rotor inlet velocity triangle design case and CFD results with design and adjusted nozzle metal angles.

Table 4.7: Rotor inlet flow property comparison between the design case and the CFD results after the volute and nozzles with design and adjusted nozzle trailing edge metal angles.

\begin{tabular}{lccccl}
\hline \hline Parameter & & Design & CFD & CFD \\
& & & $\beta_{3^{\prime}}=-72.11^{\circ}$ & $\beta_{3^{\prime}}=-72.66^{\circ}$ & \\
\hline Total temperature & $T_{04}$ & 631.25 & 630.57 & 630.47 & $\mathrm{~K}$ \\
Total pressure & $P_{04}$ & 23290 & 22899 & 22868 & $\mathrm{kPa}$ \\
Static density & $\rho_{4}$ & 148.19 & 150.00 & 144.70 & $\mathrm{~kg} / \mathrm{m}^{3}$ \\
\hline \hline
\end{tabular}


The desired rotor inlet relative flow angle of $\beta_{4}=-20^{\circ}$ (equivalent to $\beta_{4, C F D}=-20.3^{\circ}$ at the CFD evaluation radius), was achieved with a nozzle trailing edge metal angle of $\beta_{3^{\prime}}=$ $-72.66^{\circ}$. The rotor inlet velocity triangle resulting from $\beta_{3^{\prime}}=-72.66^{\circ}$ is also shown in Figure 4.23, where it can be seen that the relative flow angle matches the design value well. The change in the nozzle geometry from $\beta_{3^{\prime}}=-72.11^{\circ}$ to $\beta_{3^{\prime}}=-72.66^{\circ}$ was not significant in terms of the throat width which decreased from $8.6 \mathrm{~mm}$ to $8.4 \mathrm{~mm}$ and the chord length which increased from $46.4 \mathrm{~mm}$ to $47.0 \mathrm{~mm}$, therefore the nozzle design recommendations used were still valid and the design was not iterated upon further.

Overall, the one-dimensional nozzle design methods resulted in a reasonable rotor inlet velocity triangle. The adjusted nozzle row was able to take the flow provided by the volute and deliver it to the rotor at the desired relative flow angle.

\subsubsection{Rotor Metal Angle Adjustment}

With the nozzle metal angles and rotor shape and geometry finalized, the rotor trailing edge metal angle should be adjusted to account for deviation at the rotor outlet. Zero swirl at the rotor outlet is desired to reduce losses in the downstream components, but the flow is likely to deviate from the metal angle at the rotor trailing edge. This trailing edge deviation was expected due to a combination of three-dimensional flow effects including inertial effects and diffusion as the flow leaves the rotating blade path as well as the tip leakage flow which has a large component in the tangential direction (Dixon \& Hall, 2013). The meanline rotor outlet velocity triangle based on the CFD results with the one-dimensional design metal angles $\left(\beta_{5^{\prime}}=55.91^{\circ}\right)$ is shown in Figure 4.24 in comparison to the designed velocity triangle. Again, for the comparison of the velocity triangles, the design values are the meanline values from the preliminary design whereas the velocities obtained from the CFD results are the area-averaged values over the whole boundary. It is seen that the area-averaged absolute velocity, $C_{5}$, does not have zero swirl, with an angle of $\alpha_{5}=12.1^{\circ}$. In addition to downstream losses, the swirl seen at the rotor outlet means that, according to Equation 2.9 and Equation 2.11, the power delivered by the rotor would be less or more than required if the tangential component is positive or negative, respectively. The area-averaged rotor outlet swirl was found to be 
substantially reduced to $\alpha_{5}=0.8^{\circ}$ after adjusting the meanline trailing edge metal angle to $\beta_{5^{\prime}}=57.91^{\circ}$. Since, the mass flow rate is specified as a boundary condition at the rotor outlet and the rotor outlet annulus geometry is constant, it would be expected that the meridional velocity for the design case and CFD cases would be the same, but the difference that is seen may be attributed to the difference in flow properties similar to what was seen when adjusting the nozzle metal angles. Table 4.8 compares the flow properties at the rotor outlet for the design case and the CFD cases.

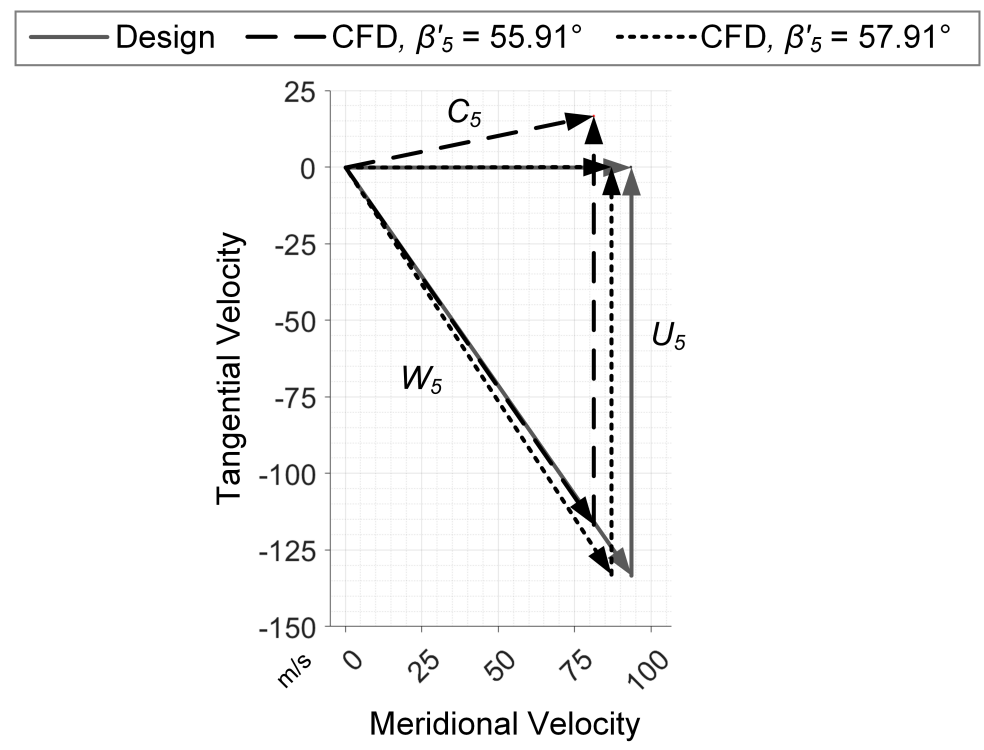

Figure 4.24: Comparison of rotor outlet velocity triangle design case and CFD results with design and adjusted rotor trailing edge metal angles.

Table 4.8: Rotor outlet flow property comparison between the design case and the CFD results with design and adjusted rotor trailing edge metal angles.

\begin{tabular}{lccccl}
\hline \hline Parameter & Design & $\begin{array}{c}\text { CFD } \\
\beta_{5^{\prime}}=55.91^{\circ}\end{array}$ & $\begin{array}{c}\text { CFD } \\
\beta_{5^{\prime}}=57.91^{\circ}\end{array}$ & Units \\
\hline Total temperature & $T_{05}$ & 535.70 & 544.98 & 537.31 & $\mathrm{~K}$ \\
Total pressure & $P_{05}$ & 8970 & 10579 & 9787 & $\mathrm{kPa}$ \\
Static density & $\rho_{5}$ & 89.09 & 104.00 & 97.25 & $\mathrm{~kg} / \mathrm{m}^{3}$ \\
\hline \hline
\end{tabular}




\subsection{On-Design Results}

The volute, nozzle, and rotor adjustments and improvements presented in the previous sections were shown to improve the performance in relative terms. However, due to the fact that the one-dimensional design was completed with an estimated isentropic efficiency and the volute and nozzles were assumed to be lossless, it is important to ensure that the performance of the three-dimensional design meets the requirements specified in Table 3.1. The final steadystate CFD results are based on a simulation with the same setup as in Section 4.6 with a single nozzle passage and a single rotor passage and nozzle inlet conditions provided by the

volute simulations. A comparison between the thermodynamic state points and performance parameters from the CFD results and the designed values is presented in Table 4.9 where a noticeable difference can be seen for some parameters. Mass averaging was used to obtain the average total conditions presented here and area averaging was used for static properties. The quoted efficiency values were obtained based on Equation 2.12 using enthalpy values obtained from REFPROP at the turbine inlet and outlet temperatures and pressures from the CFD results to avoid any interpolation errors that may have come from the use of RGP tables within the CFX post processing.

The CFD results indicate that the designed turbine is able to deliver the required power output at the design speed, mass flow, and inlet conditions, which was the main design requirement. This means that in terms of the on-design results the design is validated. However, there are noticeable differences between the CFD results and the intended design which should be addressed. First, the losses through the volute and nozzle should be investigated. The total pressure loss coefficients through these components fall within the range suggested by Japikse \& Baines (1994) as common for successful designs. Both $K$ values are actually on the lower side of the suggested range (discussed in Section 2.6.1), meaning that the losses are low. It is believed that these values may increase if the more accurate SST turbulence model could be applied to these simulations because it would capture the boundary layer and the associated profile losses better. Refining the computational meshes to allow use of the SST model and then using the SST model for similar CFD simulations is certainly something to investigate 
Table 4.9: Comparison of final steady-state CFD results with design case in terms of thermodynamic state points and performance.

\begin{tabular}{|c|c|c|c|c|}
\hline Parameter & & Design & CFD & Units \\
\hline \multicolumn{5}{|c|}{ Thermodynamic state points } \\
\hline Volute inlet total temperature & $T_{01}$ & 631.25 & 631.25 & $\mathrm{~K}$ \\
\hline Volute inlet total pressure & $P_{01}$ & 23290 & 23290 & $\mathrm{kPa}$ \\
\hline Nozzle inlet total temperature & $T_{02}$ & 631.25 & 630.95 & $\mathrm{~K}$ \\
\hline Nozzle inlet total pressure & $P_{02}$ & 23290 & 23149 & $\mathrm{kPa}$ \\
\hline Rotor inlet total temperature & $T_{04}$ & 631.25 & 630.47 & K \\
\hline Rotor inlet total pressure & $P_{04}$ & 23290 & 22868 & $\mathrm{kPa}$ \\
\hline Rotor outlet total temperature & $T_{05}$ & 535.70 & 537.31 & $\mathrm{~K}$ \\
\hline Rotor outlet total pressure & $P_{05}$ & 8970 & 9787 & $\mathrm{kPa}$ \\
\hline \multicolumn{5}{|c|}{ Velocities } \\
\hline Nozzle inlet absolute velocity & $C_{3}$ & 109.40 & 110.23 & $\mathrm{~m} / \mathrm{s}$ \\
\hline Nozzle inlet absolute flow angle & $\alpha_{3}$ & 57.2 & 56.8 & $\circ$ \\
\hline Rotor inlet absolute velocity & $C_{4}$ & 295.07 & 295.25 & $\mathrm{~m} / \mathrm{s}$ \\
\hline Rotor inlet absolute flow angle & $\alpha_{4}$ & 72.1 & 71.6 & $\circ$ \\
\hline Rotor inlet relative velocity & $W_{4}$ & 96.67 & 99.70 & $\mathrm{~m} / \mathrm{s}$ \\
\hline Rotor inlet relative flow angle & $\beta_{4}$ & -20.3 & -20.3 & $\circ$ \\
\hline Rotor outlet absolute velocity & $C_{5}$ & 93.40 & 89.62 & $\mathrm{~m} / \mathrm{s}$ \\
\hline Rotor outlet absolute flow angle & $\alpha_{5}$ & 0.0 & 0.8 & $\circ$ \\
\hline \multicolumn{5}{|c|}{ Performance } \\
\hline Mass flow rate & $\dot{m}$ & 170 & 170 & $\mathrm{~kg} / \mathrm{s}$ \\
\hline$R P M$ & & 21000 & 21000 & rpm \\
\hline Volute total pressure loss coefficient & $K_{V}$ & 0.00 & 0.12 & \\
\hline Nozzle total pressure loss coefficient & $K_{N}$ & 0.00 & 0.04 & \\
\hline Rotor total-total isentropic efficiency & $\eta_{R t t}$ & 86 & 95 & $\%$ \\
\hline Rotor power output & $\dot{W}_{R}$ & 14.72 & 14.71 & MW \\
\hline Turbine total-total isentropic efficiency & $\eta_{t t}$ & 86 & 94 & $\%$ \\
\hline Turbine power output & $\dot{W}_{t}$ & 14.72 & 14.77 & MW \\
\hline
\end{tabular}


in the future. The difference between the designed and CFD nozzle inlet total temperature and pressure were only $0.04 \%$ and $0.6 \%$, so the assumption of zero losses through the volute used in the one-dimensional design stage is acceptable. The velocity delivered by the volute according to the CFD results was $0.77 \mathrm{~m} / \mathrm{s}$ faster than the design value at an angle $0.4^{\circ}$ less and the effects of this were discussed previously with the conclusion that the flow through the nozzle was not substantially influenced so the nozzle leading edge design was not adjusted.

The flow through the nozzles was discussed previously in Section 4.6.1 to investigate the metal angles by examining Figure 4.21 and Figure 4.22. It was seen that the nozzle leading edge has a small area of separated flow at the leading edge on the suction side due to the incidence of the flow, but corrective measures were not investigated in the present work because the effect was not found to be significant for the on-design simulations. In general, the nozzle blade loading indicates a uniform loading over the chord and a steady acceleration of the flow as the static pressure decreases. At the trailing edge, the wake causes the sudden drop in static pressure as the flow diffuses out of the blade passage as expected. The losses through the volute and nozzle combined result in differences of $0.1 \%$ and $1.8 \%$ for total temperature and total pressure at the rotor inlet when comparing the CFD results to the design values. The difference in total pressure may be of concern and may lead to the implementation of stator loss estimates in future one-dimensional design methods. The ability of the nozzle to deliver the desired rotor inlet flow has been discussed in depth in Section 4.6.1, but Table 4.9 highlights that the adjusted nozzles deliver the flow at the desired relative flow angle to the rotor despite differences in total flow properties and velocity magnitudes.

The flow through the rotor may be represented by the relative Mach number distribution at $50 \%$ span as shown in Figure 4.25 and the blade loading shown in Figure 4.26. The discontinuity in the Mach number distribution in Figure 4.25 from the stator to the rotor boundary is due to the change in reference frame. A region of low Mach number is seen after the leading edge on the pressure side, but the velocity vectors indicate that no recirculation occurs. The blade loading charts support this because the effect of the negative incidence is only seen as a slight drop in pressure on the suction side and slight increase in pressure on the pressure side at about $2 \%$ of the meridional distance. The velocity vectors after the leading 
edge reflect those presented by Japikse \& Baines (1994) where the negative incidence is seen to cause an area of low speed on the pressure side. Another area of concern seen in Figure 4.25 is on the suction side at about $40 \%$ meridional distance where another area of low Mach number is seen. This is around where the blade wrap angle increases as shown in Figure 4.3, which may indicate that the wrap angle changes too quickly in that area. The blade loading charts reflect this flow structure by the change in the slope of the pressure around $40 \%$. The result of this is that the difference between the pressure on the pressure and suction sides is smaller and thus the blade loading is lower after $40 \%$. Removing this area of low Mach number from the flow by adjusting the rotor metal angle distributions would be advised as future work. Similar to the nozzle blades, an expansion occurs at the rotor trailing edge that causes the dip in static pressure seen on the blade loading charts. The rotor efficiency of $95 \%$ from the CFD results is much higher than the estimated value used for the one-dimensional design, meaning that the rotor outlet total conditions are much higher than the design values. This high rotor efficiency again may be reduced if the SST turbulence model can be implemented in the future. The rotor Power output from the CFD results is $0.01 \mathrm{MW}$ or $0.06 \%$ less than the design value and this difference may be attributed to the difference in the absolute flow angles at the rotor inlet and rotor outlet based on Equation 2.11 and Equation 2.9. Further refinement of the nozzle and rotor metal angles would allow for the power output to match the design value, but the difference seen in the present work was accepted.

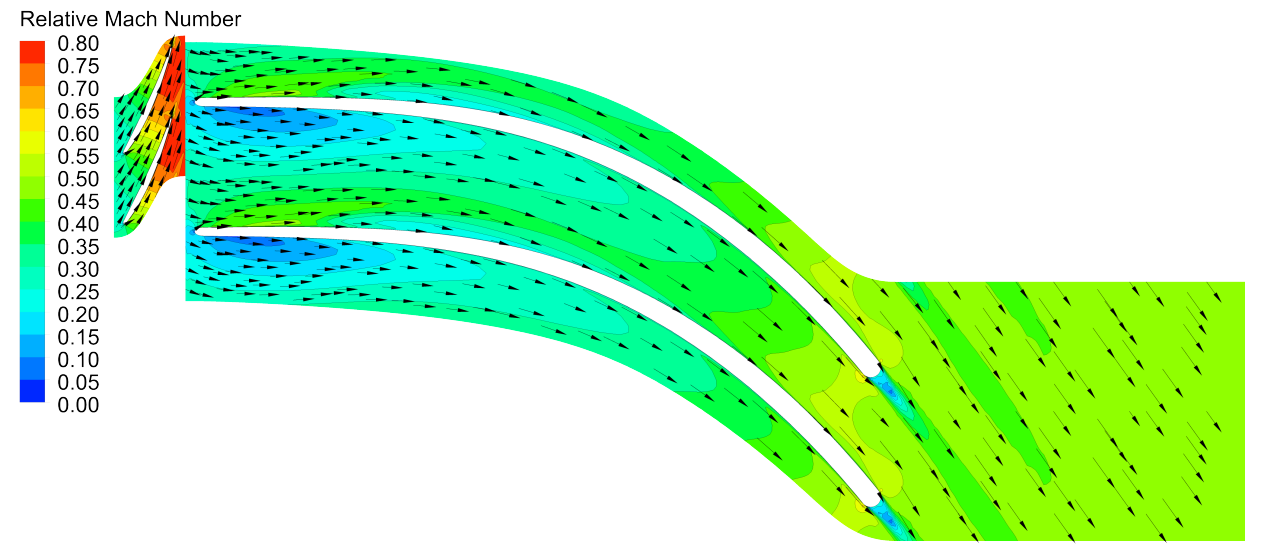

Figure 4.25: Mach number and velocity vectors through nozzles and rotor at $50 \%$ span. 


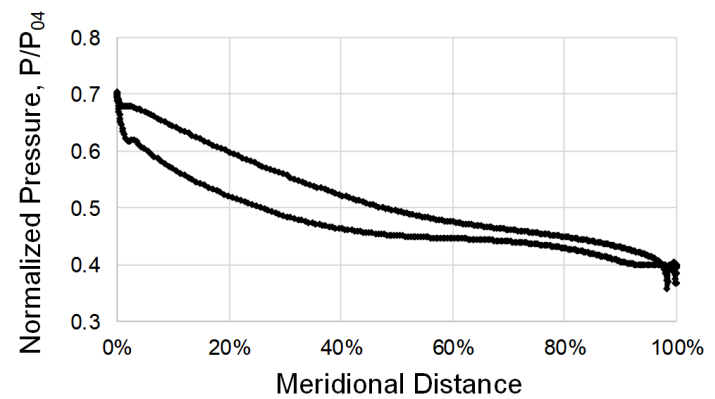

(a) $50 \%$ span.

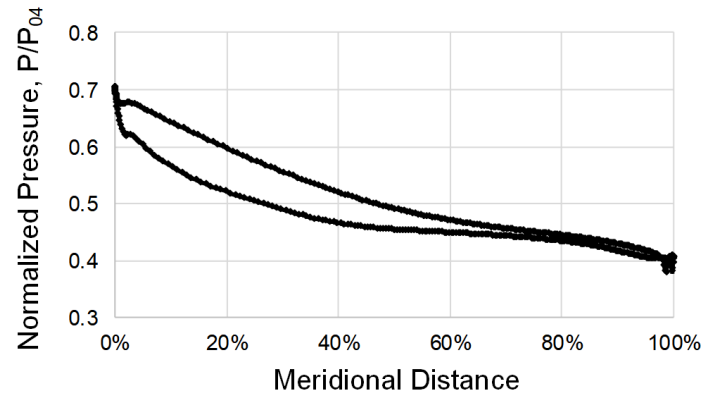

(b) $20 \%$ span.

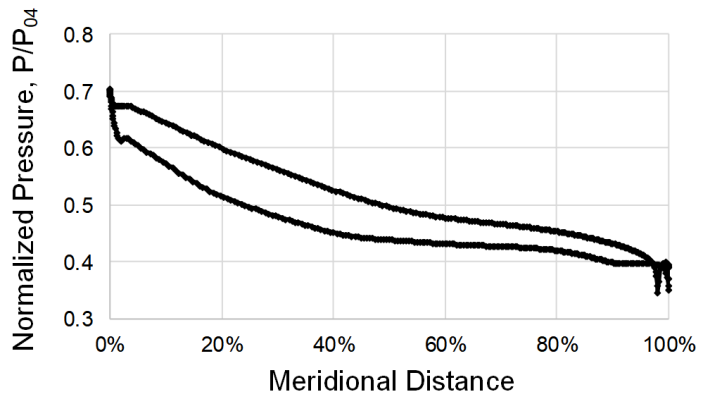

(c) $80 \%$ span.

Figure 4.26: Rotor blade loading at 20\%, 50\%, and $80 \%$ span.

Overall, the designed turbine meets the specified requirements, providing 14.71 MW with a mass flow rate of $170 \mathrm{~kg} / \mathrm{s}$ at $21000 \mathrm{rpm}$. The whole turbine efficiency of $94 \%$ was higher than the initial estimate of $86 \%$. The rotor efficiency was calculated as $95 \%$ and the volute and nozzle total pressure loss coefficients were only 0.12 and 0.04 respectively. Future work may investigate a thicker nozzle leading edge to handle the incidence better and a refined rotor wrap angle distribution to reduce the area of low Mach number seen on the suction side. Validation of the CFD results based on experimental data is not possible because experimental data for S-CO2 turbine operation at the power scale of the present work is not available. A push for experimental data is required by those investigating $\mathrm{S}-\mathrm{CO} 2$ power cycle to validate all models that may be used. 


\subsection{Importance of Real Gas Properties Implementation}

Real gas properties were implemented for the present CFD work, but it is worth understanding whether they need to be implemented for future work or if ideal gas relations are acceptable. For this reason, the final turbine design was evaluated in CFD using ideal gas properties for comparison with the results obtained using real gas properties. The simulations were setup similarly to the nozzle and rotor simulations described in Section 4.6 and with the same meshes, but with an ideal gas definition of $\mathrm{CO}_{2}$. This ideal gas definition used the NASA format to define the zero-pressure specific heat capacity $c_{\mathrm{p}_{0}}$ (ANSYS, Inc., 2017a).

As discussed in Section 2.3, the ideal gas equation of state, $\rho=P / R T$, does not accurately represent the density for some states of real gases. The percent error of the ideal gas density compared to the density obtained from REFPROP in the turbine operation region is shown in Figure 4.27. The errors at the turbine inlet, station 01, are higher than at the turbine outlet, station 05, as would be expected based on the compressibility factor, where $Z_{01}=0.995$ and $Z_{05}=0.960$. The differences in density value can result in different or erroneous fluid properties in the CFD solver, namely different velocity values. Another key difference between real gas properties and ideal gas properties is that $c_{\mathrm{p}}$ varies with both $P$ and $T$ for real gases, but only varies with $T$ for ideal gases. The effect of this difference on the accuracy of $c_{\mathrm{p}}$ in the region of operation for this turbine can be seen in Figure 4.28 where the percent error between the ideal gas and real gas definitions is plotted versus pressure and temperature. The

result of this error between the real gas and ideal gas $c_{\mathrm{p}}$ values affects the CFD calculation of the specific enthalpy, $h$, and specific entropy, $s$, directly (ANSYS, Inc., 2017b). Erroneous enthalpy and entropy values can result in the CFD solver calculating erroneous temperature, pressure, and density state points, which can then lead to erroneous velocities and other flow properties. 


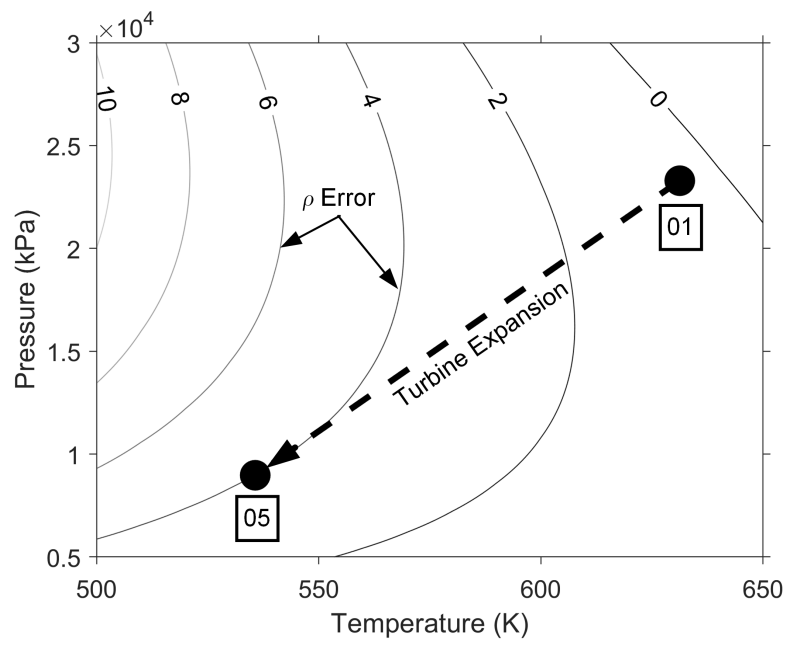

Figure 4.27: Percent error of $\rho$ for the ideal gas implementation versus REFPROP plotted against $P$ and $T$. ([REFPROP Value - Ideal Gas Value]/REFPROP Value.)

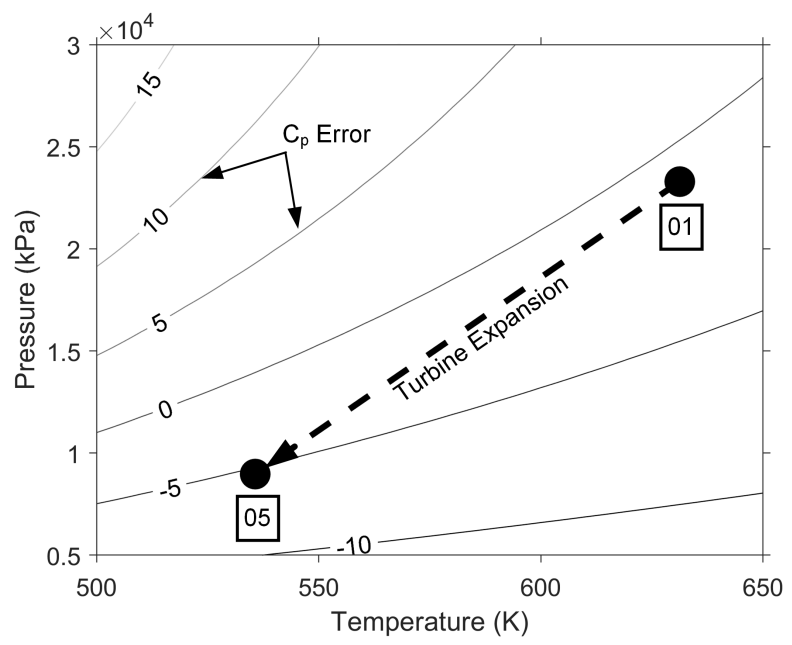

Figure 4.28: Percent error of $c_{\mathrm{p}}$ for the ideal gas implementation versus REFPROP plotted against $P$ and $T$. ([REFPROP Value - Ideal Gas Value]/REFPROP Value.)

The results of the ideal gas CFD simulations of the nozzle and rotor combination can be compared to the real gas simulations of the On-Design Results Section. Figure 4.29 and Figure 4.30 show the velocity triangles for the rotor inlet and outlet respectively, comparing the ideal gas results to the real gas results. At the rotor inlet (Figure 4.29), the nozzle trailing edge blade angle delivers the flow at the same angle in the absolute frame for both cases. The difference between the two cases is that in the ideal gas case, the magnitude of the meridional velocity component is larger than in the real gas case. This difference in meridional velocity 
magnitude can be attributed to continuity and the difference in static density. As one would expect based on Figure 4.27, the density calculated for the ideal gas case is lower than the real gas case even though the temperature and pressure state points for both cases are similar as shown in Table 4.10. The result is that the angle of the relative flow for the ideal case is $-12^{\circ}$ compared to the design value of $-20^{\circ}$ for the real gas case. A similar result is seen for the rotor outlet velocity triangle (Figure 4.30). The rotor trailing edge metal angle delivers the flow at the same angle in the relative frame for both cases. A lower calculated density for the ideal gas case results in the angle of the absolute velocity being $-6^{\circ}$ compared to the design value of $0^{\circ}$ for the real gas case due to an increased meridional velocity.

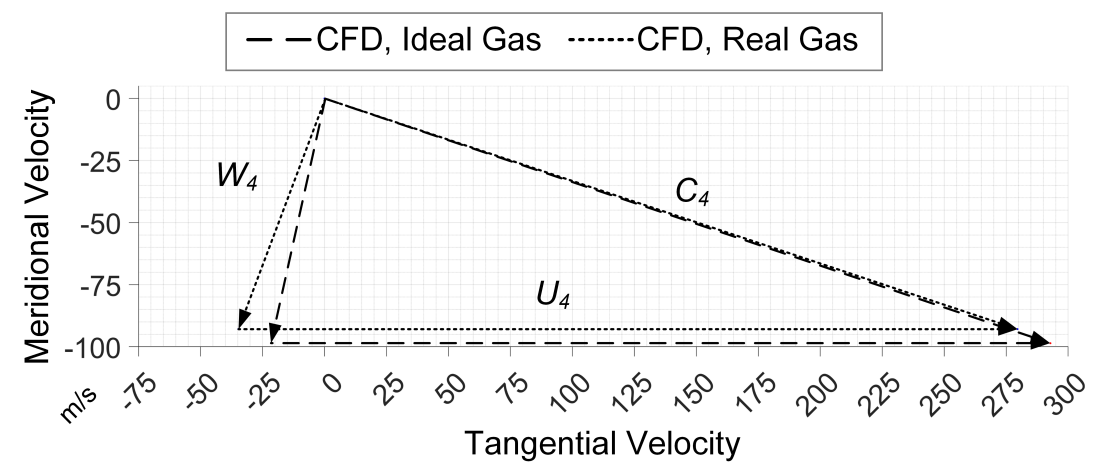

Figure 4.29: Comparison of rotor inlet velocity triangle CFD results with ideal gas and real gas properties.

Table 4.10 also compares the calculated performance in terms of the rotor efficiency and power output. It should be noted that the rotor efficiency for the ideal gas case is based on enthalpy values calculated within the CFD solver as $h=c_{p} T$ using the NASA format $c_{p}$ values which have the error shown in Figure 4.28. Nevertheless, the calculated rotor efficiency for the ideal gas case is comparable to the real gas case with only a $1 \%$ change. The rotor power output on the other hand is almost $1 \mathrm{MW}(6 \%)$ larger for the ideal gas case compared to the real gas case. This difference can be explained by Equation $2.9, \dot{W}_{t}=\dot{m}\left(h_{01}-h_{05}\right)$, because of the difference in enthalpy values due to the NASA format $c_{p}$ and it can also be explained by Equation 2.11 where the change in $U C_{\theta}$ relates to the change in work and power. Figure 4.29 and Figure 4.30 show that $C_{\theta 4}$ is more positive and $C_{\theta 5}$ is more negative for the ideal gas case. 


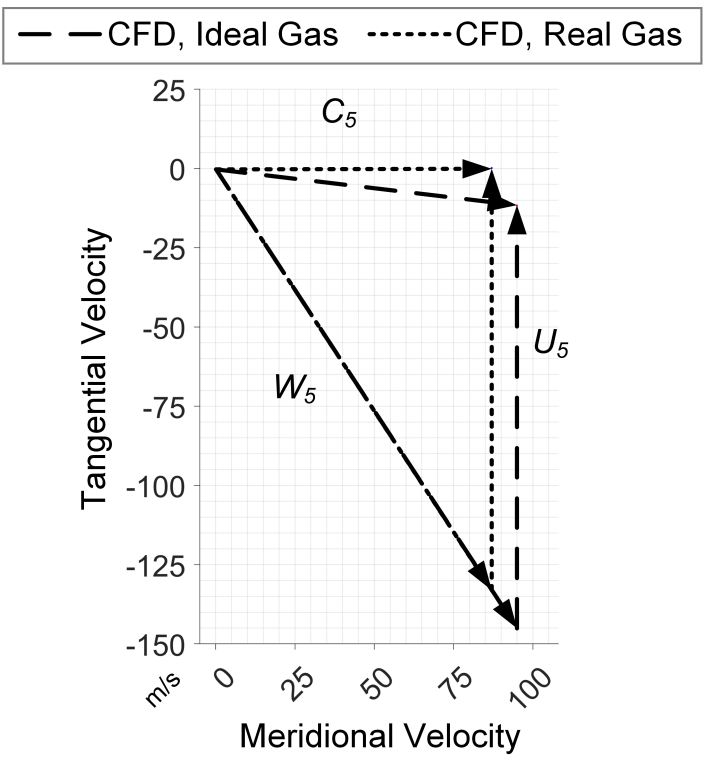

Figure 4.30: Comparison of rotor outlet velocity triangle design CFD results with ideal gas and real gas properties.

Table 4.10: Comparison of CFD results with ideal gas and real gas properties in terms of thermodynamic state points and performance.

\begin{tabular}{lcccc}
\hline \hline Parameter & \multicolumn{5}{c}{ CFD, Ideal Gas } & CFD, Real Gas & Units \\
\hline Thermodynamic state points & & \\
\hline Rotor inlet total temperature & $T_{04}$ & 630.86 & 630.47 & $\mathrm{~K}$ \\
Rotor inlet total pressure & $P_{04}$ & 22852 & 22868 & $\mathrm{kPa}$ \\
Rotor inlet static density & $\rho_{4}$ & 135.66 & 144.70 & $\mathrm{~kg} / \mathrm{m}^{3}$ \\
Rotor outlet total temperature & $T_{05}$ & 544.72 & 537.31 & $\mathrm{~K}$ \\
Rotor outlet total pressure & $P_{05}$ & 9520 & 9787 & $\mathrm{kPa}$ \\
Rotor outlet static density & $\rho_{5}$ & 88.77 & 97.25 & $\mathrm{~kg} / \mathrm{m}^{3}$ \\
\hline & Performance & & \\
\hline Rotor total-total isentropic efficiency & $\eta_{R t t}$ & 94 & 95 & $\%$ \\
Rotor power output & $\dot{W}_{R}$ & 15.62 & 14.71 & $\mathrm{MW}$ \\
\hline \hline
\end{tabular}

With the same blade speeds, $U_{4}$ and $U_{5}$, for both cases, the $\Delta U C_{\theta}$ is larger for the ideal gas case indicating more work and power.

This comparison between using ideal gas or real gas properties for this turbine design indicates that real gas properties should be implemented whenever possible for CFD solvers. 
If only ideal gas properties were used, the nozzle and rotor trailing edge metal angles would be adjusted to different angles to achieve the desired rotor inlet relative flow angle and rotor outlet absolute flow angle respectively. This would mean that the turbine would be designed to the incorrect flow conditions and have non-negligible incidences and deviations. Real gas properties should be implemented in CFD solvers to capture the finer details of the turbine design. 


\section{Chapter 5}

\section{Off-Design Modelling}

\subsection{Introduction}

This chapter covers the off-design modelling of the S-CO2 turbine developed in the present work. CFD simulations similar to those performed in the previous chapter were performed to characterize the turbine by developing performance maps. The mass flow rate and the rotational speed were varied whereas the turbine inlet pressure and temperature were held constant, using non-dimensional parameters to relate the findings to the different inlet conditions. In this chapter, the methods used to apply CFD to develop the turbine performance maps and a discussion of the results are presented.

\subsection{Turbine Performance Parameters and Characteristics}

Many authors have applied similitude theory and dimensional analysis to characterize a specific turbomachine's performance and allow for comparison between similar machines. The dimensional analysis used in the present work was based on Sjolander (2017). In the present work, the performance of the turbine may be represented by the efficiency, $\eta_{t t}$, the power output, $\dot{W}_{t}$, and the total enthalpy rise, $\Delta h_{0}$. The independent variables for the present nonideal gas turbine include the rotational speed, $N$, the rotor tip diameter, $D_{4}$, the mass flow rate, $\dot{m}$, and the turbine inlet total flow properties. The properties of the flow at the inlet can be represented by the total temperature, $T_{01}$, the total density, $\rho_{01}$, the dynamic viscosity, 
$\mu_{01}$, the specific gas constant, $R$, the compressibility factor, $Z$, and the isentropic exponent, $n$. The isentropic exponent was shown by Baltadjiev et al. (2015) to relate the specific heat ratio, $\gamma$, to $Z$ by Equation 5.1. It can be shown that the speed of sound in a non-ideal gas may be expressed as Equation 5.2 with $Z n$ replacing $\gamma$ in the ideal gas form of $a=\sqrt{\gamma R T}$.

$$
\begin{aligned}
& n=\frac{\gamma Z}{Z-P\left(\frac{\partial Z}{\partial P}\right)_{T}} \\
& a=\sqrt{Z n R T}
\end{aligned}
$$

Buckingham Pi Theorem indicates that there are five criteria of similarity for a dimensional analysis of the present scenario, of which two are $Z$ and $n$ because they are non-dimensional already. A dimensional analysis leads to the derivation of a Speed Parameter, Flow Parameter, and Reynolds Number (Viscosity Parameter). The dependent variables $\dot{W}_{t}$ and $\Delta h_{0}$ may be non-dimensionalized into a Power Coefficient and an Enthalpy Drop Parameter, and $\eta_{t t}$ is already non-dimensional. When the combination of $R T_{01}$ results from the dimensional analysis, it was replaced with $a_{01}$ which relates to $R T_{01}$ and the criteria of similarity $Z$ and $n$ according to Equation 5.2. So, $Z$ and $n$ are not "true" independent parameters because they only appear as part of the speed of sound. Sjolander (2017) discusses dimensional analysis for real gas turbomachinery in greater detail. More work may be required to ensure that highly variant real gas properties can be captured and characterized through these (or another set of) non-dimensional parameters.

$$
\begin{array}{r}
\text { Speed Parameter : } \frac{N D_{4}}{a_{01}} \\
\text { Flow Parameter }: \frac{\dot{m}}{\rho_{01} D_{4}^{2} a_{01}} \\
\text { Reynolds Number }: \frac{\rho_{01} N D_{4}^{2}}{\mu_{01}} \\
\text { Enthalpy Drop Parameter }: \frac{\Delta h_{0}}{a_{01}^{2}} \\
\text { Power Coefficient }: \frac{\dot{W}}{\rho_{01} N^{3} D_{4}^{5}}
\end{array}
$$


The Speed Parameter in the present form is the rotor blade tip Mach number, $U_{4} / a_{01}$, and the Flow Parameter is effectively the rotor inlet meridional Mach number, $C_{m 4} / a_{01}$. This realization allows further understanding of the physical meaning of the presented parameters. Similarly, the Enthalpy Drop Parameter relates to the familiar pressure ratio, $P_{01} / P_{05}$, through the efficiency. The advantage of using the Enthalpy Drop Parameter instead of the pressure ratio as a dependent parameter is that it is independent of changes in $Z, n$, and $\gamma$, whereas pressure ratio is not. This allows for the developed performance maps to be applicable for multiple fluids.

\subsection{Off-Design Modelling Methods}

The performance characterization for the present turbine was completed in CFD. Ideally, the CFD results would be validated based on experimental data, but this is not possible because experimental data for a S-CO2 turbine operating at the power scale of the present work is not available. The CFD methods applied in Section 4.4 and Section 4.6 were used for the off-design modelling as well, using the same meshes discussed in Section 4.3.

Volute off-design simulations were performed with constant inlet total pressure and temperature specified at the inlet as $P_{01}=23290 \mathrm{kPa}$ and $T_{01}=631.25 \mathrm{~K}$ with the flow direction normal to the cross-section. The volute inlet turbulence was also held constant at $k=0.2$ $\mathrm{m}^{2} / \mathrm{s}^{2}$ and $\epsilon=500 \mathrm{~m}^{2} / \mathrm{s}^{3}$. The only varied boundary condition was the outlet mass flow rate. Mass flow rates of $70,90,130,170,190$, and $210 \mathrm{~kg} / \mathrm{s}$ were simulated through the volute.

The nozzle and rotor were simulated in tandem with a stage (mixing plane) interface between the stationary and rotating frame. The rotor speed was varied to $80 \%, 100 \%$, and $120 \%$ of the design RPM. Again, the mass flow rate was specified at the rotor outlet except at higher pressure ratios where a rotor outlet static pressure is specified to resolve the asymptotic behaviour usually seen where only small changes in mass flow rate or Speed Parameter are associated with large changes in pressure ratio or Enthalpy Drop Parameter. The nozzle inlet conditions were obtained from the volute off-design simulations at the same location used in Section 4.6 by importing profile data from the volute simulation corresponding to the 
desired mass flow rate. When a rotor outlet static pressure was specified instead of a mass flow rate, the resulting mass flow rate occasionally did not match one of the simulated volute mass flow rates. The closest mass flow rate was used in these cases. The effect of this on the results was assumed to be negligible because only small changes in volute outlet (nozzle inlet) total properties due to changes in volute mass flow were seen as shown in Table 5.1. The variation in nozzle inlet flow angle is more pronounced due to the change in mass flow rate as the meridional velocity component will change directly according to continuity and Equation 3.72. If the tangential velocity is assumed to be constant, an increase in mass flow rate and meridional velocity would decrease the flow angle and vice versa. The effect of this was also assumed to be negligible but may be something to investigate or correct in the future.

Table 5.1: Percent change of average total properties and change in flow angle at volute outlet (Station 2) for different mass flow rates with the design value $(170 \mathrm{~kg} / \mathrm{s})$ as the baseline.

\begin{tabular}{lccc}
\hline \hline$\dot{m}[\mathrm{~kg} / \mathrm{s}]$ & $P_{02}$ & $T_{02}$ & $\alpha_{2}$ \\
\hline 70 & $0.23 \%$ & $0.01 \%$ & $0.36^{\circ}$ \\
90 & $0.20 \%$ & $0.01 \%$ & $0.34^{\circ}$ \\
130 & $0.11 \%$ & $0.01 \%$ & $0.21^{\circ}$ \\
170 (Baseline) & - & - & - \\
190 & $-0.11 \%$ & $-0.01 \%$ & $-0.16^{\circ}$ \\
210 & $-0.23 \%$ & $-0.01 \%$ & $-0.35^{\circ}$ \\
\hline \hline
\end{tabular}

\subsection{Results}

The turbine performance maps resulting from the off-design CFD simulations are shown in Figure 5.1 and Figure 5.2. The curves fit to the presented maps are further explained in Appendix E. These fitted curves are extrapolated past the limits of the CFD data further into the stall and choke regions of the machine where the extrapolation may not be the most accurate. The range of the CFD data was limited by convergence of the simulations because 
as the turbine began operating in the stall and choke regions, more unsteady and computationally challenging flow structures developed. In the stall and choke regions convergence was occasionally limited to root mean square residuals of $10^{-5}$ instead of the desired $10^{-6}$, but solutions were accepted if the other monitored parameters converged. Figure 5.1 shows the turbine performance in terms of Flow Parameter versus Enthalpy Drop Parameter. Choking causes the asymptotic relation at high Flow Parameter values where the turbine cannot handle higher mass flow rates. At lower flow rates, decreases in Flow Parameter are shown to cause only small changes in Enthalpy Drop Parameter due to stalling of the rotor blades.

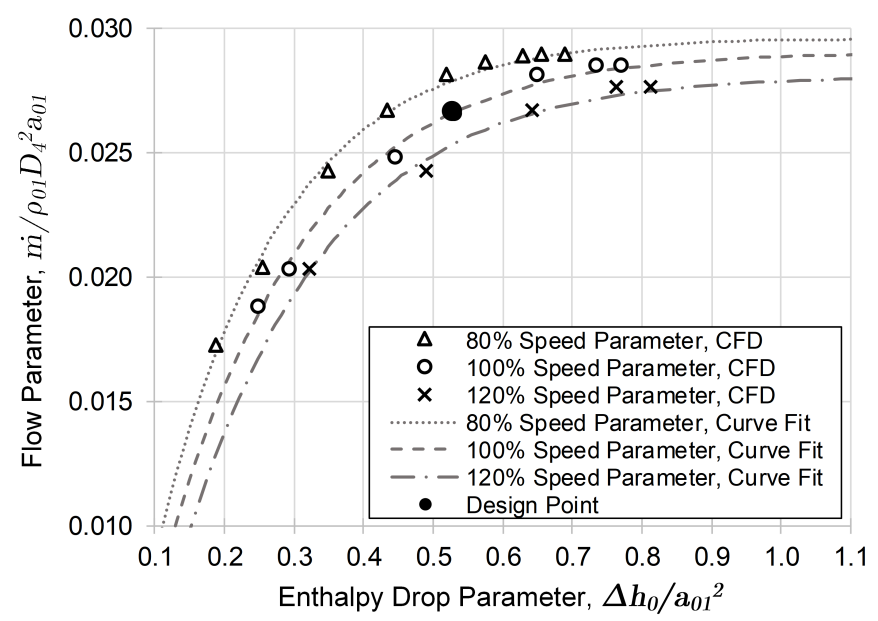

Figure 5.1: Flow Parameter versus Enthalpy Drop Parameter for Speed Parameters of 80\%, $100 \%$, and $120 \%$ of the design speed.

The different choking mass flow rates shown in Figure 5.1 indicate that rotor choking is occurring. If only nozzle choking was present, the speed lines would converge at the same choking mass flow rate, independent of the rotational speed. To further investigate this, the average Mach numbers at the nozzle trailing edge, rotor leading edge, and rotor trailing edge are shown in Figure 5.3. The average properties presented were evaluated outside of the blade passage for each location as shown in Table 5.2 (reproduced from Table 4.6). This simplification means that the listed Mach numbers may be lower than, and do not necessarily represent, the throat Mach number, but the trend was seen to be similar. Based on Figure 5.3a, supersonic Mach numbers at the nozzle trailing edge and therefore nozzle choking is only seen for the $80 \%$ 


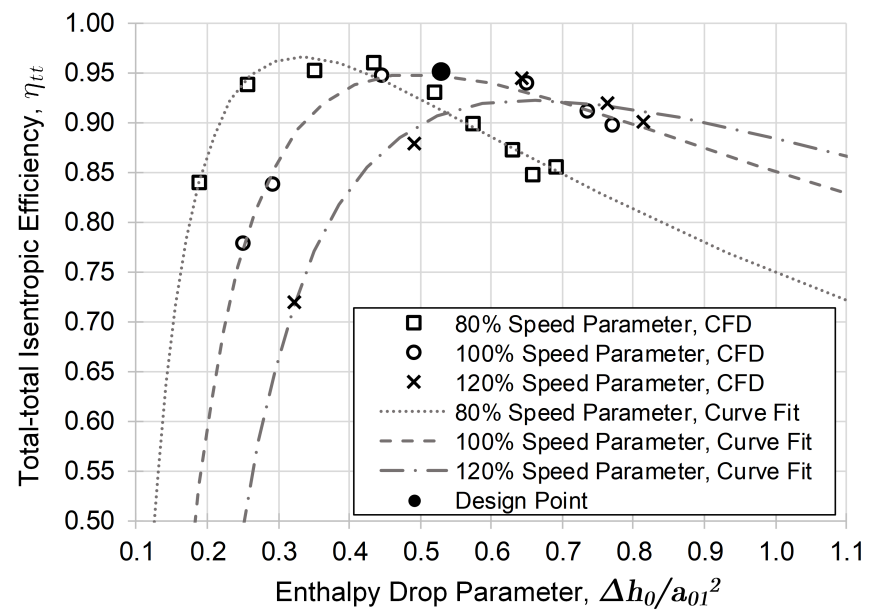

Figure 5.2: Total-total Isentropic Efficiency versus Enthalpy Drop Parameter for Speed Parameters of $80 \%, 100 \%$, and $120 \%$ of the design speed.

Speed Parameter case. Figure 5.3c shows that choking occurs at the rotor trailing edge for all Speed Parameter cases due to the higher rotational speed and relative velocity. Figure 5.3b confirms that supersonic flow and choking only occurs at the rotor trailing edge and not at the leading edge for the $100 \%$ and $120 \%$ Speed Parameter cases.

Table 5.2: Property evaluation locations offset from blade leading and trailing edges.

\begin{tabular}{lccccc}
\hline \hline Location & \multicolumn{2}{c}{ Physical Location } & \multicolumn{2}{c}{ CFD Evaluation Location } \\
& Axial, $z$ & Radial, $r$ & Axial, $z$ & Radial, $r$ \\
\hline Nozzle Trailing Edge & 0 to $b_{4}$ & $r_{3}$ & 0 to $b_{4}$ & $0.99 r_{3}$ \\
Rotor Leading Edge & 0 to $b_{4}$ & $r_{4}$ & 0 to $b_{4}$ & $1.01 r_{4}$ \\
Rotor Trailing Edge & $L_{R}$ & $r_{h 5}$ to $r_{t 5}$ & $1.05 L_{R}$ & $r_{h 5}$ to $r_{t 5}$ \\
\hline \hline
\end{tabular}

The difference between nozzle and rotor choking is shown in Figure 5.4. At the low rotational speed, $80 \%$ Speed Parameter, the nozzle is seen to choke first as shown by the supersonic flow through the nozzle throat. The rotor trailing edge Mach number at this low rotational speed remains subsonic. Conversely, at the higher rotational speed, 120\% Speed Parameter, the rotor trailing edge chokes before the nozzles. 


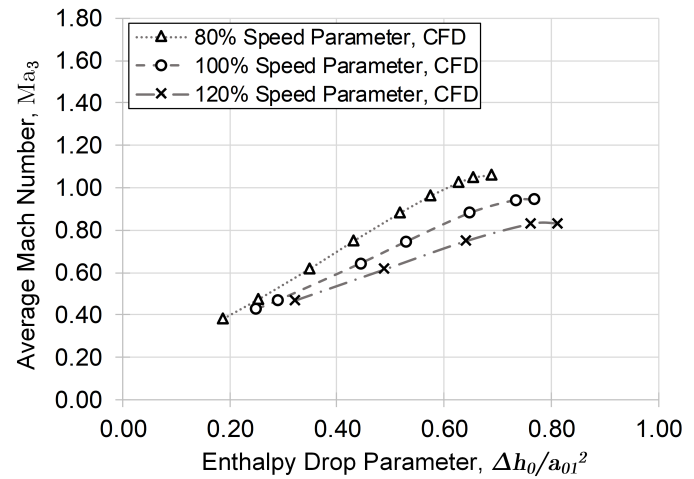

(a) Nozzle trailing edge.

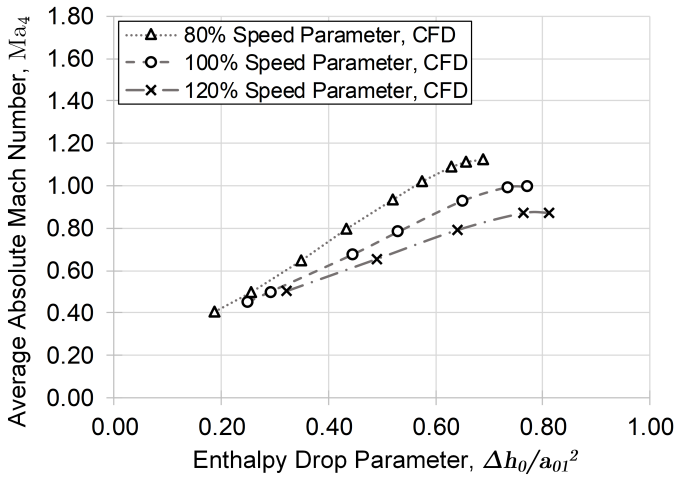

(b) Rotor leading edge.

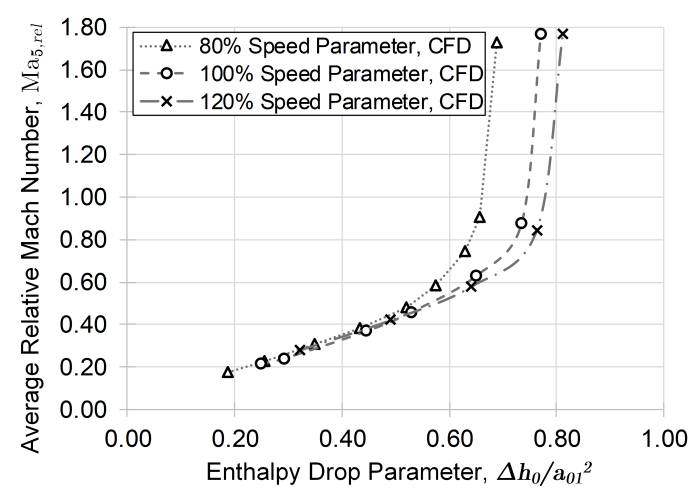

(c) Rotor trailing edge.

Figure 5.3: Average Mach number at critical locations versus Enthalpy Drop Parameter for Speed Parameters of $80 \%, 100 \%$, and $120 \%$ of the design speed.

Stalling was seen on the rotor blades at lower Flow Parameter values indicated by the smaller changes in Enthalpy Drop Parameter with large changes in Flow Parameter in Figure 5.1. The stalling can be attributed to the change in rotor inlet relative flow angle due to the change in mass flow rate and therefore meridional velocity. Figure 5.5 shows the increase in $\beta_{4}$ with Flow Parameter and shows that the angle can quickly deviate from the design value. The effect of off-design $\beta_{4}$ is shown in Figure 5.6 where the higher negative incidence is seen to cause a larger separation zone and recirculation at the rotor leading edge. 

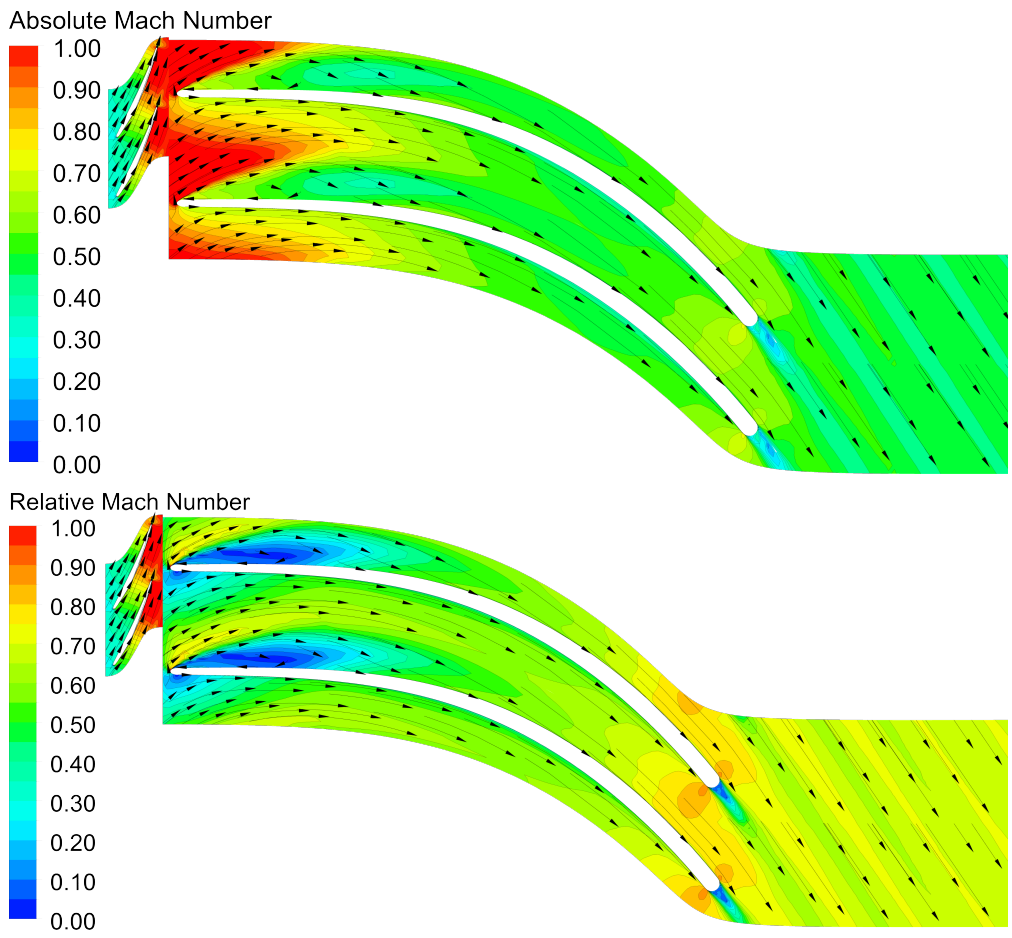

(a) Nozzle choking example. Flow Parameter $=2.86 \times 10^{-2}$, Speed Parameter $=80 \%$ of design.
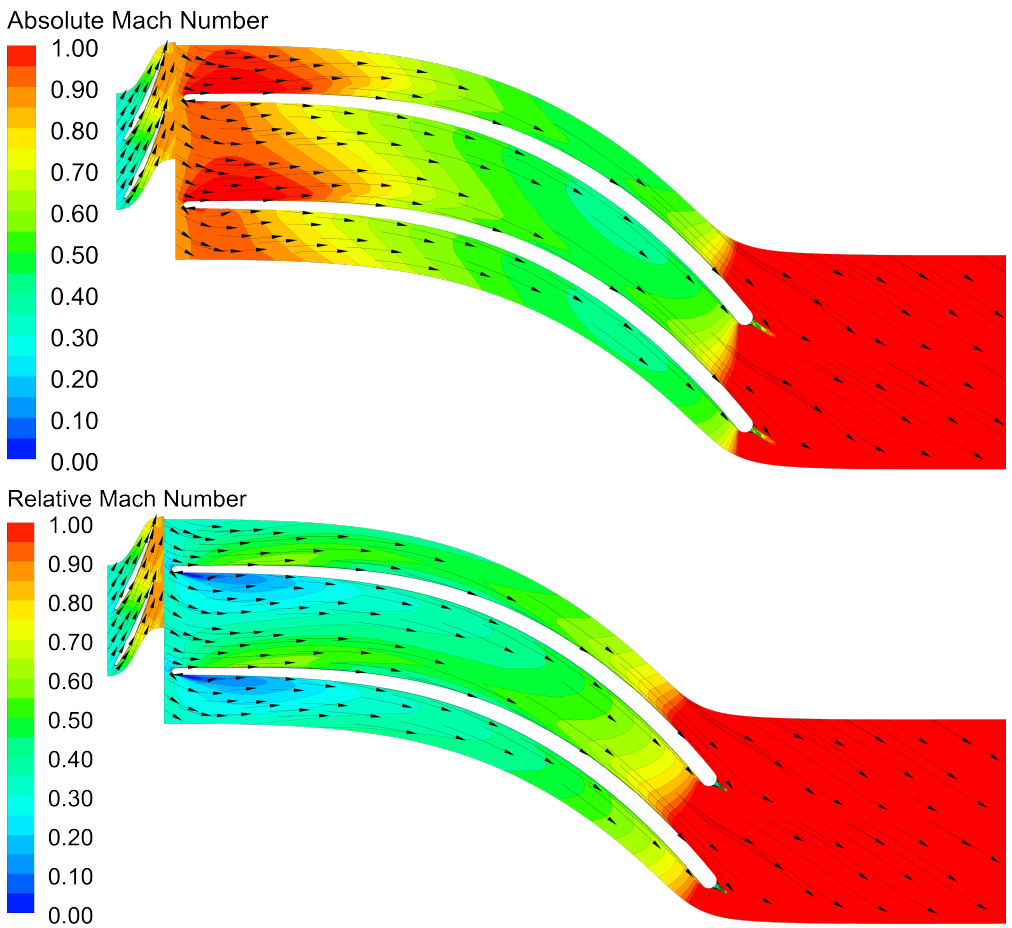

(b) Rotor choking example. Flow Parameter $=2.77 \times 10^{-2}$, Speed Parameter $=120 \%$ of design.

Figure 5.4: Mach number and velocity vectors through nozzles and rotor at $50 \%$ span for choking Flow Parameters and Speed Parameters of $80 \%$ and $120 \%$ of the design speed. 


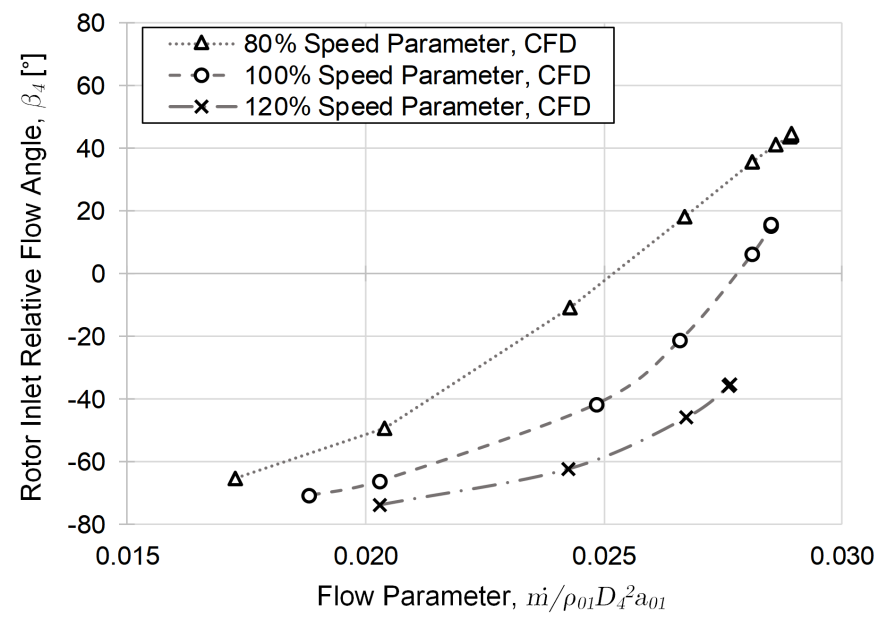

Figure 5.5: Rotor inlet relative flow angle versus Flow Parameter for Speed Parameters of $80 \%, 100 \%$, and $120 \%$ of the design speed.

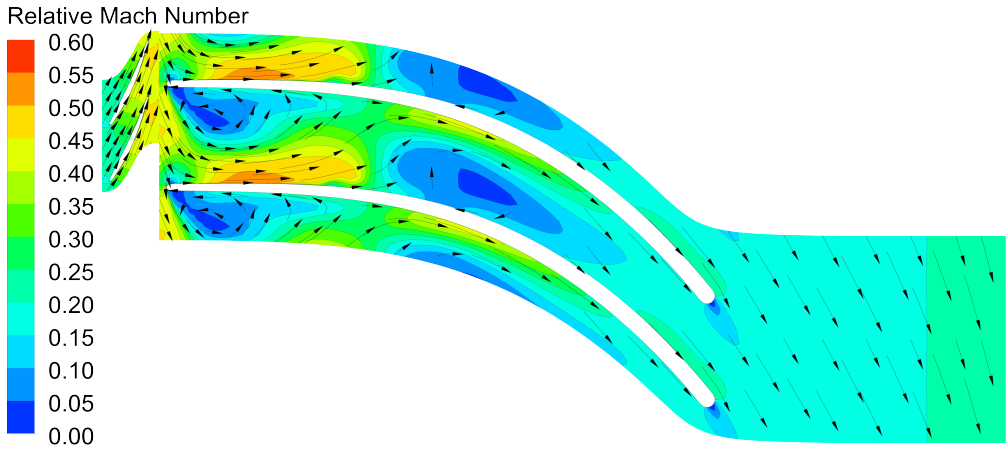

Figure 5.6: Mach number and velocity vectors through nozzles and rotor at $50 \%$ span for a Flow Parameter of $1.88 \times 10^{-2}$ and design Speed Parameter. 
Overall, the off-design performance of the designed turbine may need to be refined in the future to allow for a wider range of operation with respect to mass flow rate. Currently, at the design speed, the rotor chokes at a mass flow rate only $7 \%$ higher than design. At the design speed and a mass flow rate $24 \%$ less than design, the efficiency drops by $11 \%$ due to stalling of the rotor blades. Depending on the cycle design and operation these off-design limitations may not be acceptable, so this is something to be further investigated in the future. 


\section{Chapter 6}

\section{Summary, Conclusions, and Recommendations}

\subsection{Summary}

The aerodynamic design of a radial inflow turbine for a $10 \mathrm{MW}_{\mathrm{e}}$ supercritical $\mathrm{CO}_{2}$ power cycle has been completed in the present work. A one-dimensional design method was applied, using recommendations from a variety of literature sources. The one-dimensional design was then implemented in three-dimensions to allow for evaluation with CFD at the design point and to characterize the design in terms of off-design performance. The key contribution of the present work was the development of one-dimensional design methods and computational evaluation methods for radial turbines operating with non-ideal gases.

The potential benefits of S-CO2 power cycles was discussed in the Literature Review. Specifically, the high density of $\mathrm{CO}_{2}$ near the critical point allows for low compression work requirements and therefore higher potential cycle efficiencies. This benefit of operating in the supercritical region also presents difficulties when modelling the fluid properties due to the large variations that can be seen with only small changes in state. The Literature Review also covered these interesting fluid property variations and methods for modelling them.

A radial inflow turbine was selected for the cycle in the present work and a one-dimensional design methodology for them was detailed in Chapter 3. Following the discussion in the Literature Review on the importance of using accurate fluid property models, the onedimensional design method was developed to account for real gas properties through equations of state. Specifically, REFPROP was used when an equation of state was required in 
the one-dimensional design method in the present work. One-dimensional volute, nozzle, and rotor designs were developed for the present work and presented in Chapter 3 also.

The one-dimensional volute, nozzle, and rotor designs were implemented in threedimensions in Chapter 4 to allow for evaluation in CFD. Fluid properties were modelled in CFD using refined RGP tables created with data from REFPROP. The volute design was improved in terms of the Swirl Coefficient, choosing the value that provided the best performance according to the CFD results. Similarly, the nozzle and rotor metal angles and camberlines were varied and evaluated in CFD to match the desired design velocity triangle and determine which delivered the best performance. The final design turbine was shown to exceed the expected performance with an efficiency of $94 \%$ while delivering the desired power output at the design operating conditions.

Finally, the off-design performance of the designed turbine was characterized through CFD by developing performance maps. Plots of non-dimensional parameters including Isentropic Efficiency, Flow Parameter, Enthalpy Drop Parameter, and Speed Parameter were presented based on CFD data points with adequate fitted curves to extrapolate the trends. The choking and stalling regions for the present design were identified showing that the present design may be limited in terms of off-design operating range.

\subsection{Conclusions and Recommendations}

The present work provided a complete turbine aerodynamic design that met the design requirements. However, there are some limitations to the work that should be addressed with recommendations for future work.

The present one-dimensional design method provides a quick design that could meet the given design requirements. However, implementation of loss models should allow for better prediction of efficiency before requiring full CFD evaluation, so this should be pursued in the future. Loss models were not implemented in the present work to allow for quicker preliminary design and due to uncertainty about the accuracy of models from literature for non-ideal gases 
such as S-CO2. It is recommended that the accuracy of radial turbine loss models be further evaluated and developed for non-ideal gases.

Although S-CO2 turbines may operate with compressibility factors close to ideal gas $(0.95$ to 0.99 ), the implementation of real gas properties when evaluating them with CFD was shown to be necessary to capture detailed aspects of the turbine design. The nozzle and rotor trailing edge deviation was found to be affected when only ideal gas properties were used in the CFD solver resulting in rotor incidence and exit swirl angle error of $8^{\circ}$ and $6^{\circ}$ respectively. It is assumed that the same limitations of ideal gas seen in the comparison of the CFD results would be applicable at the preliminary design stage as well. If erroneous density values are used in the preliminary design stage due to using ideal gas properties, the result would be an erroneous rotor and turbine sizing. However, the magnitude and impact of these errors has not been confirmed and could be further investigated.

The use of the $k-\epsilon$ model in the present work may have resulted in unrealistically high efficiency values if the scalable wall functions underestimated the viscous effects in the boundary layer. The $k-\omega$ SST model is said to better resolve the boundary layer, however, it was difficult to implement it in the present work due to computational difficulties from mesh requirements. Therefore, it is recommended that the effect of using the $k-\epsilon$ model versus more accurate models such as the $k-\omega$ SST model should be characterized in terms of effect on calculated performance. This would require more work creating a mesh with acceptable $y^{+}$values that could be resolved computationally. This may require more computing power than what was available for the present work or the creation of a custom mesh, in ICEM for example, with acceptable refinement near walls but less in the freestream to limit the mesh size.

The examined range of off-design operation for the designed turbine should be extended to further understand the choking and stalling limitations. The present work was limited in that only Flow Parameters in the range of $70 \%$ to $107 \%$ of the design value were examined. The choking and stalling limits should be investigated further to determine if any design changes can be implemented to extend the off-design operation range. The nozzle blade thickness and camber profiles may be further investigated to improve the handling of off-design incidence. 
Also, further evaluation of the best rotor leading edge incidence may lead to better performance by limiting the separation at the leading edge and potentially delaying stall onset.

Finally, the lack of experimental data for $\mathrm{S}-\mathrm{CO} 2$ turbine operation at the scale of the present work meant that the computational results could not be validated. It is strongly recommended that a full-scale S-CO2 power cycle test facility be developed to allow for validation of designs methods such as those presented here. 


\section{References}

Allison, T., Moore, J., Pelton, R., Wilkes, J., \& Ertas, B. (2017). Turbomachinery. In K. Brun, P. Friedman, \& R. Dennis (Eds.), Fundamentals and Applications of Supercritical Carbon Dioxide $\left(\mathrm{sCO}_{2}\right)$ Based Power Cycles (pp. 147-215). Woodhead Publishing.

Ameli, A., Afzalifar, A., Turunen-Saaresti, T., \& Backman, J. (2017). Effects of real gas model accuracy and operating conditions on supercritical co2 compressor performance and flow field. In ASME Turbo Expo 2017: Turbomachinery Technical Conference and Exposition (pp. V009T38A011-V009T38A011).: American Society of Mechanical Engineers.

Angelino, G. (1968). Carbon dioxide condensation cycles for power production. Journal of Engineering for Power, 90(3), 287-295.

Angelino, G. (1969). Real gas effects in carbon dioxide cycles. In ASME 1969 Gas Turbine Conference and Products Show: American Society of Mechanical Engineers.

ANSYS, Inc. (2017a). ANSYS CFX-Solver Modeling Guide. ANSYS, Inc., release 18.2 edition. ANSYS, Inc. (2017b). ANSYS CFX-Solver Theory Guide. ANSYS, Inc., release 18.2 edition.

Aungier, R. H. (2006). Turbine Aerodynamics: Axial-Flow and Radial-Flow Turbine Design and Analysis. ASME Press.

Baltadjiev, N. D., Lettieri, C., \& Spakovszky, Z. S. (2015). An investigation of real gas effects in supercritical co2 centrifugal compressors. Journal of Turbomachinery, 137(9), 091003.

Bidkar, R., Mann, A., Singh, R., Sevincer, E., Cich, S., Day, M., Kulhanek, C., Thatte, A., Peter, A., Hofer, D., et al. (2016a). Conceptual designs of 50 mwe and 450 mwe supercritical 
co2 turbomachinery trains for power generation from coal. part 1: cycle and turbine. In 5th International Symposium-Supercritical CO, volume 2 (pp. 28-31).

Bidkar, R., Musgrove, G., Day, M., Kulhanek, C., Allison, T., Peter, A., Hofer, D., \& Moore, J. (2016b). Conceptual designs of 50 mwe and 450 mwe supercritical co2 turbomachinery trains for power generation from coal. part 2: compressors. In 5th International SymposiumSupercritical CO, volume 2 (pp. 28-31).

Cengel, Y. A. (2007). Introduction to thermodynamics and heat transfer. McGraw-Hill New York.

Crespi, F., Gavagnin, G., Sánchez, D., \& Martínez, G. (2017). Supercritical carbon dioxide cycles for power generation: A review. Journal of Applied Energy, 195, 152-183.

Csanady, G. T. (1964). Theory of turbomachines. McGraw-Hill.

Dixon, S. L. \& Hall, C. (2013). Fluid mechanics and thermodynamics of turbomachinery. Butterworth-Heinemann, 6th edition.

Dostal, V. (2004). A supercritical carbon dioxide cycle for next generation nuclear reactors. Ph.D. thesis, Massachusetts Institute of Technology, Department of Nuclear Engineering.

Feher, E. G. (1968a). Investigation of supercritical (feher) cycle. Astropower Laboratory, Missile 8 Space Systems Division.

Feher, E. G. (1968b). The supercritical thermodynamic power cycle. Energy conversion, 8(2), $85-90$.

Fiaschi, D., Innocenti, G., Manfrida, G., \& Maraschiello, F. (2016). Design of micro radial turboexpanders for orc power cycles: From 0d to 3d. Applied Thermal Engineering, 99, 402-410.

Finney, B. \& Jacobs, M. (2010). Carbon dioxide pressure-temperature phase diagram. Creative Commons via Wikimedia. Available at: https://upload.wikimedia.org/wikipedia/ 
commons/1/13/Carbon_dioxide_pressure-temperature_phase_diagram.svg. Accessed August 22, 2017.

Friedman, P. \& Anderson, M. (2017). Thermodynamics. In K. Brun, P. Friedman, \& R. Dennis (Eds.), Fundamentals and Applications of Supercritical Carbon Dioxide ( $\left.\mathrm{sCO}_{2}\right)$ Based Power Cycles (pp. 41-66). Woodhead Publishing.

Futral, S. M. \& Holeski, D. E. (1970). Experimental results of varying the blade-shroud clearance in a 6.02-inch radial-inflow turbine. Technical report, NASA.

Galindo, J., Hoyas, S., Fajardo, P., \& Navarro, R. (2013). Set-up analysis and optimization of cfd simulations for radial turbines. Engineering Applications of Computational Fluid Mechanics, 7(4), 441-460.

Held, T. J. (2015). Supercritical co2 cycles for gas turbine combined cycle power plants. Power Gen International.

Iwai, Y., Itoh, M., Morisawa, Y., Suzuki, S., Cusano, D., \& Harris, M. (2015). Development approach to the combustor of gas turbine for oxy-fuel, supercritical co2 cycle. In ASME Turbo Expo 2015: Turbine Technical Conference and Exposition (pp. V009T36A013V009T36A013).: American Society of Mechanical Engineers.

Jahn, I. (2015). Radial Inflow Turbine Meshing Rev. 1. Technical report, School of Mechanical and Mining Engineering, The University of Queensland. Mechanical Engineering Technical Report 2015/03.

Japikse, D. \& Baines, N. C. (1994). Introduction to Turbomachinery. Oxford University Press, Oxford.

Johnson, G. A., McDowell, M. W., OâĂŹConnor, G. M., Sonwane, C. G., \& Subbaraman, G. (2012). Supercritical co2 cycle development at pratt and whitney rocketdyne. In ASME turbo expo 2012: turbine technical conference and exposition (pp. 1015-1024).: American Society of Mechanical Engineers. 
Jones, A. C. (1994). Design and test of a small, high pressure ratio radial turbine. In ASME 1994 International Gas Turbine and Aeroengine Congress and Exposition (pp. V001T01A045-V001T01A045).: American Society of Mechanical Engineers.

Kimball, K. J. \& Clementoni, E. M. (2012). Supercritical carbon dioxide brayton power cycle development overview. In ASME Turbo Expo 2012: Turbine Technical Conference and Exposition (pp. 931-940).: American Society of Mechanical Engineers.

Kofskey, M. G. \& Wasserbauer, C. A. (1966). Experimental performance evaluation of a radial-inflow turbine over a range of specific speeds. Technical report, NASA. NASA TN D-3742.

Lemmon, E. W., Huber, M. L., \& McLinden, M. O. (2013). NIST Standard Reference Database 23: Reference Fluid Thermodynamic and Transport Properties-REFPROP, Version 9.1, National Institute of Standards and Technology.

MacLean, C. A. (2012). Effects of real fluid properties on axial turbine meanline design and off-design analysis. M.A.Sc. thesis, Carleton University.

Mazzoccoli, M., Bosio, B., \& Arato, E. (2012). Analysis and comparison of equations-of-state with p- $\rho$-t experimental data for co2 and co2-mixture pipeline transport. Energy Procedia, $23,274-283$.

McDowell, M., Eastland, A., Huang, M., \& Swingler, C. (2015). Advanced turbomachinery for sco2 power cycles. In Atlanta, GA: University Turbine Systems Research Workshop.

Moore, J., Brun, K., Evans, N., \& Kalra, C. (2015). Development of 1 mwe supercritical co2 test loop. In ASME Turbo Expo 2015: Turbine Technical Conference and Exposition (pp. V009T36A015-V009T36A015).: American Society of Mechanical Engineers.

Musgrove, G. \& Wright, S. (2017). Introduction and background. In K. Brun, P. Friedman, \& R. Dennis (Eds.), Fundamentals and Applications of Supercritical Carbon Dioxide $\left(\mathrm{sCO}_{2}\right)$ Based Power Cycles (pp. 1-22). Woodhead Publishing. 
Nusbaum, W. \& Kofskey, M. (1969). Cold performance evaluation of 4.97-inch radial-inflow turbine design for single-shaft Brayton cycle space-power system. Technical report, NASA. NASA TN D-5090.

Poling, B. E., Prausnitz, J. M., O'Connell, J. P., et al. (2001). The properties of gases and liquids, vol. 5. McGraw-Hill, New York.

Qi, J., Reddell, T., Qin, K., Hooman, K., \& Jahn, I. H. (2017). Supercritical co2 radial turbine design performance as a function of turbine size parameters. Journal of Turbomachinery, 139(8), 081008.

Qiang, X., Teng, J., \& Du, Z. (2010). Influence of various volute designs on volute overall performance. Journal of thermal science, 19(6), 505-513.

Rohlik, H. E. (1968). Analytical determination of radial inflow turbine design geometry for maximum efficiency. Technical report, NASA. NASA TN D-4384.

Sauret, E. (2012). Open design of high pressure ratio radial-inflow turbine for academic validation. In ASME 2012 International Mechanical Engineering Congress and Exposition (pp. 3183-3197).: American Society of Mechanical Engineers.

Sauret, E. \& Gu, Y. (2014). Three-dimensional off-design numerical analysis of an organic rankine cycle radial-inflow turbine. Applied Energy, 135, 202-211.

Saxena, S., Mallina, R., Moraga, F., \& Hofer, D. (2017). Numerical approach for real gas simulations: Part ii: Flow simulation for supercritical co2 centrifugal compressor. In ASME Turbo Expo 201\%: Turbomachinery Technical Conference and Exposition (pp. V009T38A005-V009T38A005).: American Society of Mechanical Engineers.

Simpson, A., Spence, S., \& Watterson, J. (2013). Numerical and experimental study of the performance effects of varying vaneless space and vane solidity in radial turbine stators. Journal of Turbomachinery, 135(3), 031001.

Sjolander, S. (2017). Mech 4305: Fluid machinery - supplementary course notes. Department of Mechanical and Aerospace Engineering, CArleton University. 
Span, R. \& Wagner, W. (1996). A new equation of state for carbon dioxide covering the fluid region from the triple-point temperature to $1100 \mathrm{k}$ at pressures up to $800 \mathrm{mpa}$. Journal of physical and chemical reference data, 25(6), 1509-1596.

Thimsen, D. (2013). Program on technology innovation: modified brayton cycle for use in coal-fired power plants. Electric Power Research Institute (EPRI).

Watanabe, I., Ariga, I., \& Mashimo, T. (1971). Effect of dimensional parameters of impellers on performance characteristics of a radial-inflow turbine. Journal of Engineering for Power, 93(1), 81-102.

White, C. W. \& Weiland, N. T. (2017). Evaluation of property methods for modeling directsupercritical co2 power cycles. In ASME Turbo Expo 201\%: Turbomachinery Technical Conference and Exposition (pp. V009T38A017-V009T38A017).: American Society of Mechanical Engineers.

White, M. (2015). The design and analysis of radial inflow turbines implemented within low temperature organic Rankine cycles. Ph.D. thesis, City University London.

Whitfield, A. (1989). The preliminary design of radial inflow turbines. In ASME 1989 International Gas Turbine and Aeroengine Congress and Exposition (pp. V001T01A049V001T01A049).: American Society of Mechanical Engineers.

Whitfield, A. \& Baines, N. C. (1990). Design of radial turbomachines. New York, NY (USA); John Wiley and Sons Inc.

Wilkes, J. C., Wade, J., Rimpel, A., Moore, J., Swanson, E., Grieco, J., \& Brady, J. (2016). Impact of bearing clearance on measured stiffness and damping coefficients and thermal performance of a high-stiffness generation 3 foil journal bearing. In ASME Turbo Expo 2016: Turbomachinery Technical Conference and Exposition (pp. V07BT31A011-V07BT31A011).: American Society of Mechanical Engineers.

Wood, H. J. (1963). Current technology of radial-inflow turbines for compressible fluids. Journal of Engineering for Power, 85(1), 72-83. 
Wright, S. A., Radel, R. F., Vernon, M. E., Rochau, G. E., \& Pickard, P. S. (2010). Operation and analysis of a supercritical co2 brayton cycle. Sandia Report, No. SAND2010-0171.

Wright, T. \& Gerhart, P. (2009). Fluid machinery: application, selection, and design. CRC Press.

Zhang, J., Gomes, P., Zangeneh, M., \& Choo, B. (2017). Design of a centrifugal compressor stage and a radial-inflow turbine stage for a supercritical co2 recompression brayton cycle by using 3d inverse design method. In ASME Turbo Expo 2017: Turbomachinery Technical Conference and Exposition (pp. V009T38A023-V009T38A023).: American Society of Mechanical Engineers. 


\section{Appendix A}

\section{Peng-Robinson Equation of State}

The Peng-Robinson equation of state (EoS) is a cubic equation of state that allows for modelling of the real gas properties of fluids. Cubic equations of state are less computationally costly than the models in REFPROP (White, 2015), so they are favourable for CFD analysis. The Peng-Robinson EoS has been shown to perform the best out of the readily available equations of state in ANSYS CFX (Mazzoccoli et al., 2012). Cubic equations of state model a fluid's pressure, $P$, in terms of its temperature, $T$, and specific volume, $\nu=1 / \rho$ (ANSYS, Inc., 2017b). The Peng-Robinson equation of state takes the following form:

$$
P=\frac{R T}{\nu-b}-\frac{a(T)}{\nu^{2}+2 b \nu-b^{2}}
$$

where

$$
\begin{aligned}
a(T) & =a_{0}\left(1+n\left(1-\sqrt{\frac{T}{T_{c}}}\right)\right)^{2} \\
a_{0} & =0.45724 \frac{R^{2} T_{c}^{2}}{P_{c}} \\
n & =0.37464+1.54226 \omega-0.26993 \omega^{2} \\
b & =0.0778 \frac{R T_{c}}{P_{c}}
\end{aligned}
$$

where $R$ is the specific gas constant of the fluid, $T_{c}$ is the critical temperature, $P_{c}$ is the critical pressure, and $\omega$ is the acentric factor. The specific gas constant, critical temperature, critical 
pressure, and acentric factor of $\mathrm{CO}_{2}$ are $188.9 \mathrm{~J} / \mathrm{kgK}, 304.13 \mathrm{~K}, 7.377 \mathrm{MPa}$, and 0.22394, respectively (Span \& Wagner, 1996).

In order to complete the fluid property model, enthalpy and entropy relations are required. More detail on the internal calculation are available in the ANSYS CFX-Solver Theory Guide by ANSYS, Inc. (2017b). However, White (2015) described the equations in a more accessible way. The change in entropy and enthalpy can be related to the change in internal energy which may be determined by considering three thermodynamic processes from a reference point $\left(T_{r e f}, \nu_{r e f}\right)$ to any other point (White, 2015). The three thermodynamic processes to get to the desired state point $(P, T)$ are:

1. Constant temperature expansion from the reference point to an infinite volume point $\left(P_{1}=0, T_{1}=T_{\text {ref }}\right) ;$

2. Constant volume heating to the known/desired temperature $\left(P_{2}=0, T_{2}=T\right)$;

3. Constant temperature compression to the known/desired pressure $\left(P_{3}=P, T_{3}=T\right)$.

The change in internal energy as the result of these three processes is expressed as:

$$
u-u_{r e f}=\int_{\nu_{r e f}}^{\infty}\left(T\left(\frac{d P}{d T}\right)_{\mathrm{v}}-P\right) d \nu_{T_{r e f}}+\int_{T_{r e f}}^{T} c_{\mathrm{v}_{0}} d T-\int_{\nu}^{\infty}\left(T\left(\frac{d P}{d T}\right)_{\mathrm{v}}-P\right) d \nu_{T}
$$

With the internal energy calculated, the enthalpy may be solved for as:

$$
h=u+P \nu
$$

Similar to the change in internal energy, the change in entropy may be calculated as:

$$
s-s_{\text {ref }}=\int_{\nu_{r e f}}^{\infty}\left(\frac{d P}{d T}\right)_{\mathrm{v}} d \nu_{T_{r e f}}+\int_{T_{r e f}}^{T} \frac{c_{\mathrm{p}_{0}}}{T} d T-R \ln \left(\frac{P}{P_{r e f}}\right)-\int_{\nu}^{\infty}\left(\frac{d P}{d T}\right)_{\mathrm{v}} d \nu_{T}
$$

where $c_{\mathrm{p} 0}$ is the zero-pressure specific heat capacity which may be defined within ANSYS CFX by a fourth order polynomial shown in Equation A.6 with coefficients for $\mathrm{CO}_{2}$ obtained from 
Poling et al. (2001) as listed in Table A.1.

$$
c_{\mathrm{p}_{0}} / R=a_{0}+a_{1} T+a_{2} T^{2}+a_{3} T^{3}+a_{4} T^{4}
$$

Table A.1: Zero-pressure specific heat coefficients for $\mathrm{CO}_{2}$ related to Equation A.6 (Obtained from Poling et al., 2001).

\begin{tabular}{cccccc}
\hline \hline T Range & $a_{0}$ & $a_{1}$ & $a_{2}$ & $a_{3}$ & $a_{4}$ \\
\hline $50-1000 \mathrm{~K}$ & 3.259 & $1.356 \times 10^{-3}$ & $1.502 \times 10^{-5}$ & $-2.374 \times 10^{-8}$ & $1.506 \times 10^{-11}$ \\
\hline \hline
\end{tabular}

To determine if the presented implementation of the Peng-Robinson equation of state would be suitable for the present work, the Peng-Robinson calculated (from CFD results) enthalpy values were compared with the REFPROP calculated enthalpy values at the turbine's inlet and outlet pressures and temperatures from a CFD simulation. Pressure and temperature state points from 62 on-design and off-design simulations, with the ranges shown in Table A.2, were used to compare these property calculation methods. The data from the CFD simulations was averaged over the inlet and outlet boundary with mass-averaging for total conditions and area-averaging for static conditions such that excessive outliers were omitted. The enthalpy and entropy values were compared in terms of the relative change from inlet to outlet to eliminate the difference between reference state between the two property methods, whereas the static density values were compared directly at the inlet and outlet states. Statistics regarding the percentage deviation of the Peng-Robinson values compared to the REFPROP values are shown in Table A.3. The maximum percentage deviation for the static and total enthalpy values calculated with the Peng-Robinson equation of state in CFX compared to the REFPROP values was $3.9 \%$ and $8.9 \%$, and for the inlet and outlet density values was $2.0 \%$ and $3.0 \%$. The percentage deviation of the entropy with a maximum of $39 \%$ can be attributed to inadequate coefficients for the $c_{\mathrm{p} 0}$ polynomial because the entropy depends directly on $c_{\mathrm{p} 0}$ as shown in Equation A.6. This accuracy could be improved by sampling data in the turbine operating region from REFPROP and fitting a polynomial to this data, but using REFPROP to create RGP tables was easier and assumed to not be less accurate. 
Table A.2: Pressure and temperature values of the 81 state points used for comparison of Peng-Robinson and REFPROP property calculation.

\begin{tabular}{lcccccccc}
\hline \hline & $\begin{array}{c}P_{4} \\
{[\mathrm{kPa}]}\end{array}$ & $P_{5}$ & $P_{04}$ & $P_{05}$ & $T_{4}$ & $T_{5}$ & $T_{04}$ & $T_{05}$ \\
& {$[\mathrm{kPa}]$} & {$[\mathrm{kPa}]$} & {$[\mathrm{kPa}]$} & {$[\mathrm{K}]$} & {$[\mathrm{K}]$} & {$[\mathrm{K}]$} & {$[\mathrm{K}]$} \\
\hline Maximum & 22636 & 20831 & 23285 & 21141 & 628 & 620 & 631 & 622 \\
Minimum & 12253 & 1370 & 23240 & 4692 & 558 & 363 & 631 & 475 \\
Average & 17107 & 11600 & 23271 & 12465 & 594 & 548 & 631 & 561 \\
Standard Deviation & 2890 & 4365 & 12 & 3963 & 19 & 53 & 0 & 36 \\
\hline \hline
\end{tabular}

Table A.3: Statistics for percentage deviation of calculated enthalpy, entropy, and density values using the presented Peng-Robinson implementation compared to REFPROP.

\begin{tabular}{lccccc}
\hline \hline & $\Delta h$ & $\Delta h_{0}$ & $\Delta s$ & $\rho_{4}$ & $\rho_{5}$ \\
\hline Maximum & $3.9 \%$ & $8.9 \%$ & $39 \%$ & $2.0 \%$ & $3.0 \%$ \\
Average & $2.5 \%$ & $2.4 \%$ & $17 \%$ & $0.7 \%$ & $0.5 \%$ \\
Standard Deviation & $0.7 \%$ & $1.7 \%$ & $22 \%$ & $0.3 \%$ & $0.6 \%$ \\
\hline \hline
\end{tabular}




\section{Appendix B}

\section{Aungier's Rotor Meridional Profiles}

Aungier (2006) recommends specific contours for the rotor hub and shroud profiles to provide a reasonable passage area distribution and to limit the effects of curvature on the flow distribution. A circular-arc contour is used for the hub profile and a power-law contour is used for the shroud.

The circular-arc contour for the hub is defined with the largest possible circular-arc for the rotor dimensions. This circular-arc radius, $R_{C}$, is the smaller of either $\left(r_{4}-r_{h 5}\right)$ or $L_{R}$ (Equation B.1). Then a linear segment is added to either the inlet section or outlet section to complete the hub profile. These linear segments are shown as $L_{4}$ and $L_{5}$ in Figure B.1 and defined by Equation B.2 and Equation B.3.

$$
\begin{gathered}
R_{C}=\left\{\begin{array}{cc}
\left(r_{4}-r_{h 5}\right), & \left(r_{4}-r_{h 5}\right) \leq L_{R} \\
L_{R}, & \left(r_{4}-r_{h 5}\right)>L_{R}
\end{array}\right. \\
L_{4}=\left\{\begin{array}{cc}
0, & \left(r_{4}-r_{h 5}\right) \leq L_{R} \\
r_{4}-r_{h 5}-R_{C}, & \left(r_{4}-r_{h 5}\right)>L_{R}
\end{array}\right. \\
L_{5}=\left\{\begin{array}{cc}
L_{R}-R_{C}, & \left(r_{4}-r_{h 5}\right) \leq L_{R} \\
0, & \left(r_{4}-r_{h 5}\right)>L_{R}
\end{array}\right.
\end{gathered}
$$




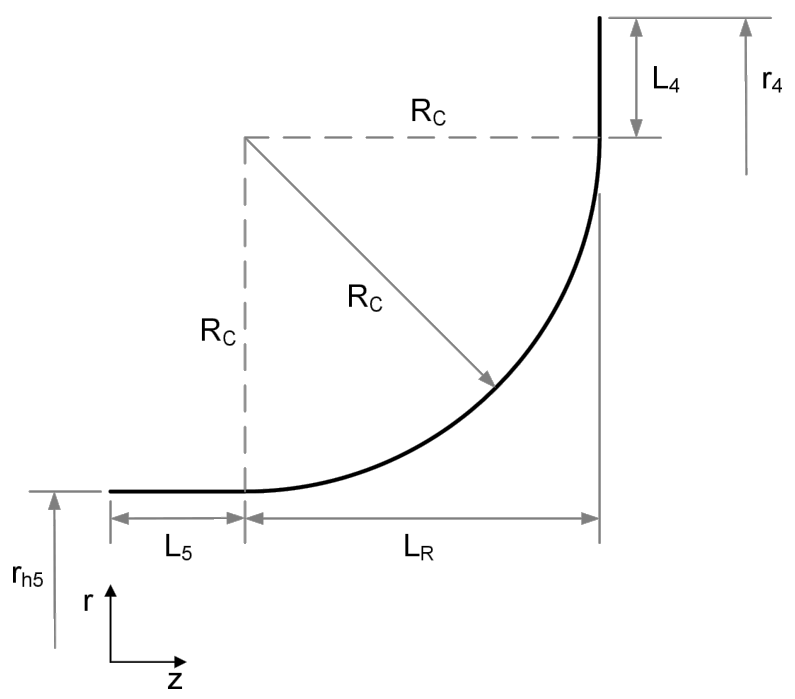

Figure B.1: Circular-arc hub contour (Adapted from Aungier, 2006).

The shroud profile is defined by a power-law contour given by Equation B.4 and shown in Figure B.2. The value of $n$ is varied from 2 to 9 to find which value results in a mean passage area, $A_{m}$, as close to the average of $A_{4}$ and $A_{5}$. The effect of the variation in $n$ on the shroud profile can be seen in Figure B.3.

$$
r(z)=r_{t 5}+\left(r_{4}-r_{t 5}\right)\left(\frac{z}{L_{R}-b_{4}}\right)^{n}
$$




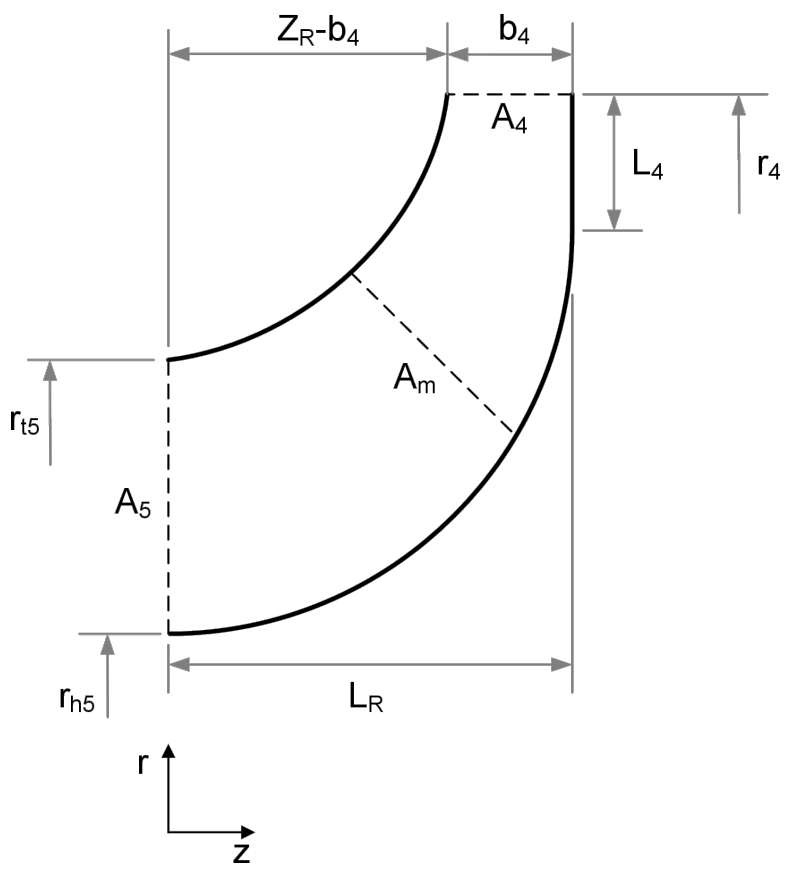

Figure B.2: Power-law shroud contour (Adapted from Aungier, 2006).
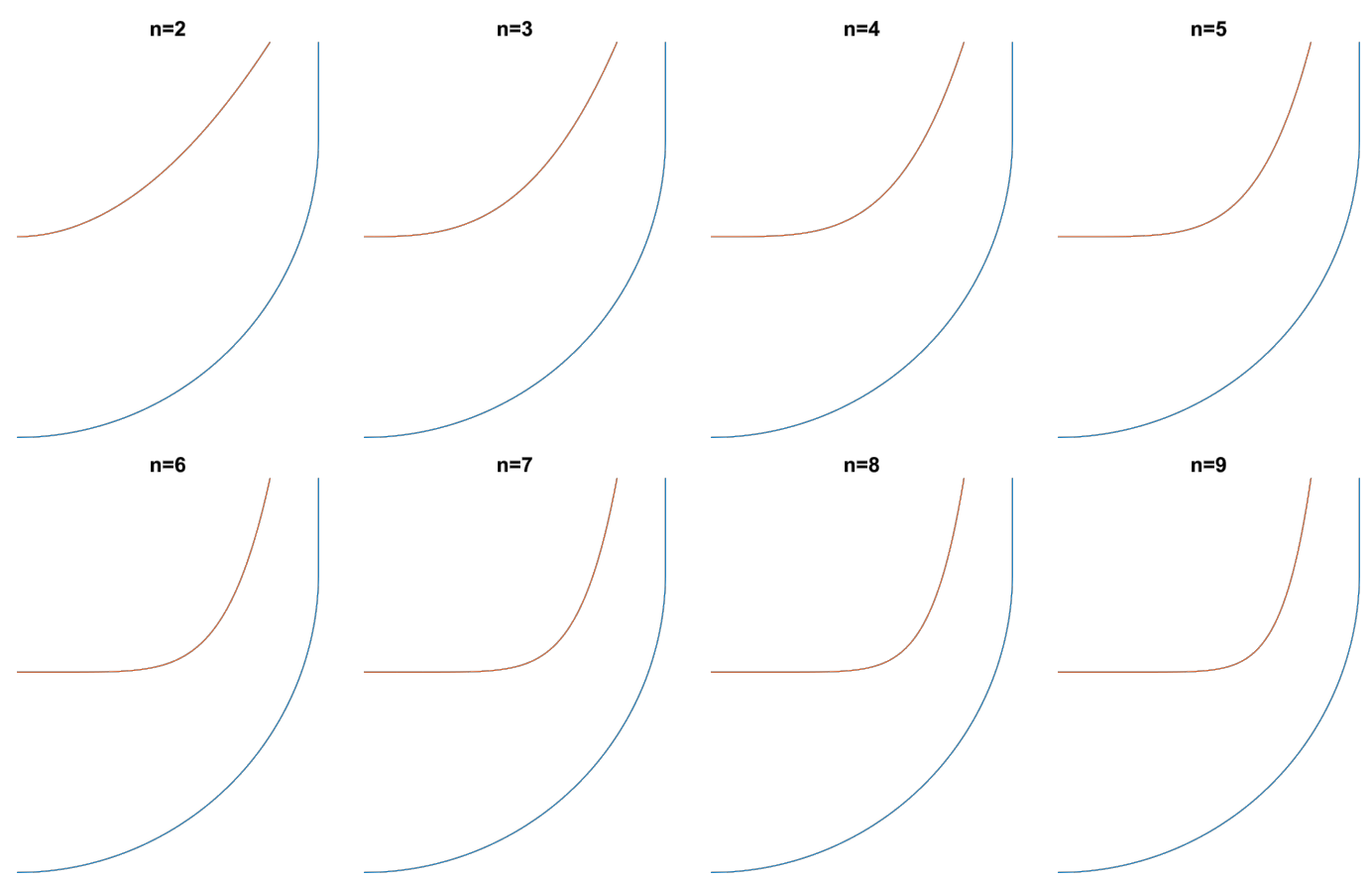

Figure B.3: Variation in shroud contour with $n$ values. 


\section{Appendix C}

\section{Computational Mesh Detail}

This Appendix includes figures to show the detail used in the computational meshes for the present work. 


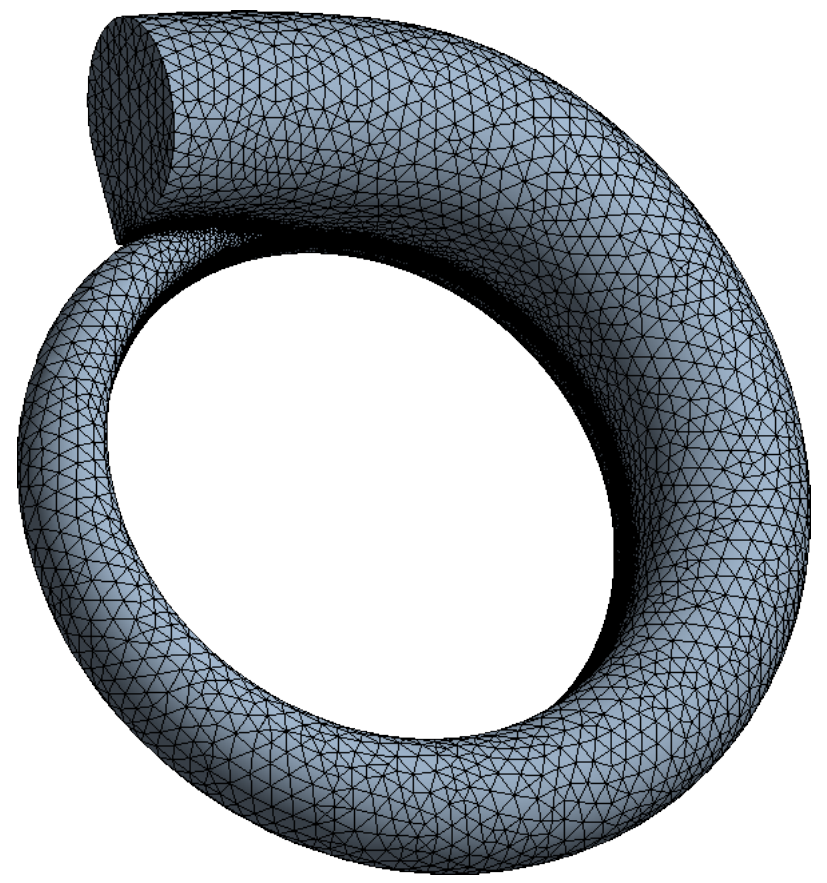

(a) Full domain.

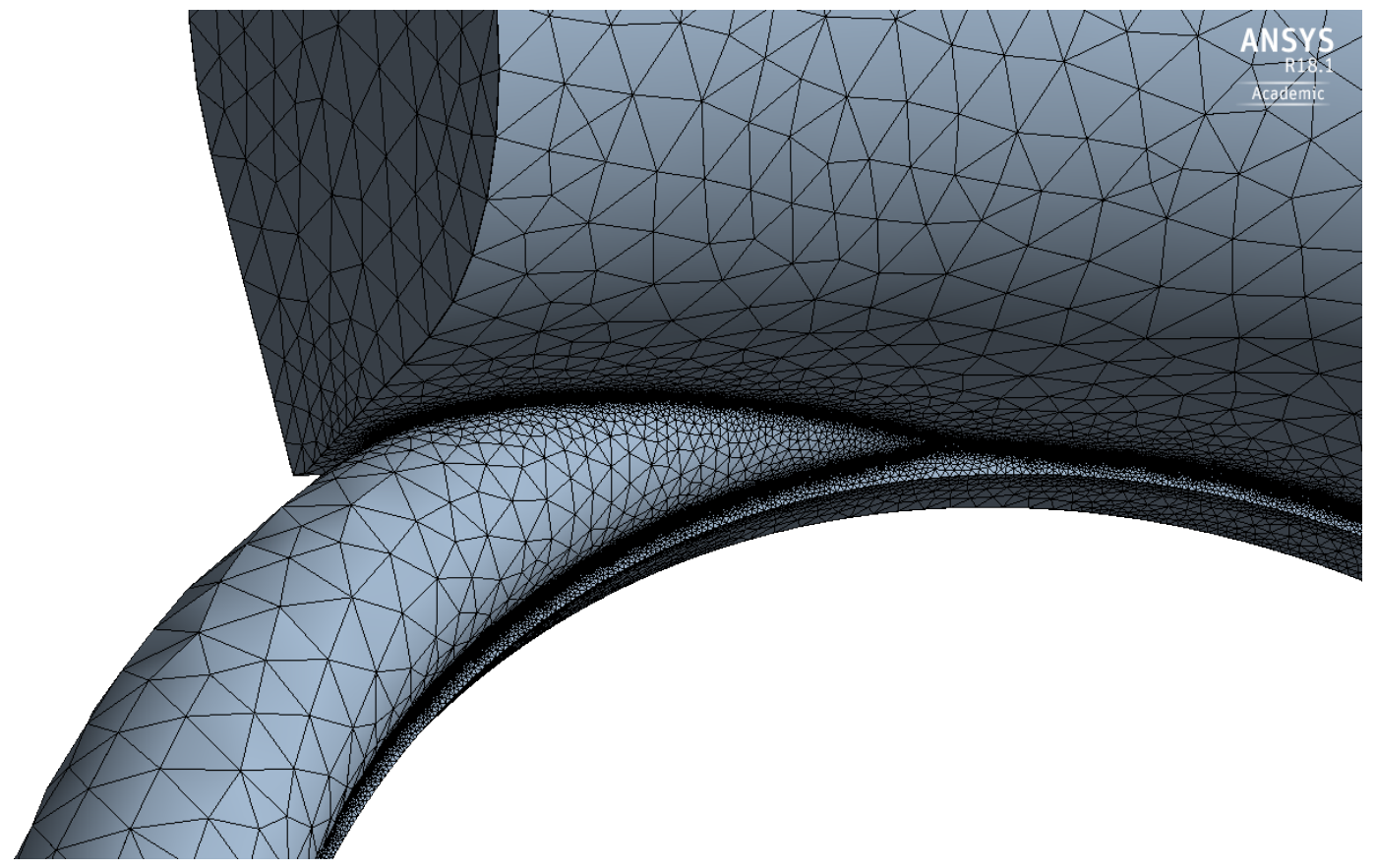

(b) Inlet and tongue location.

Figure C.1: Volute mesh detail. 


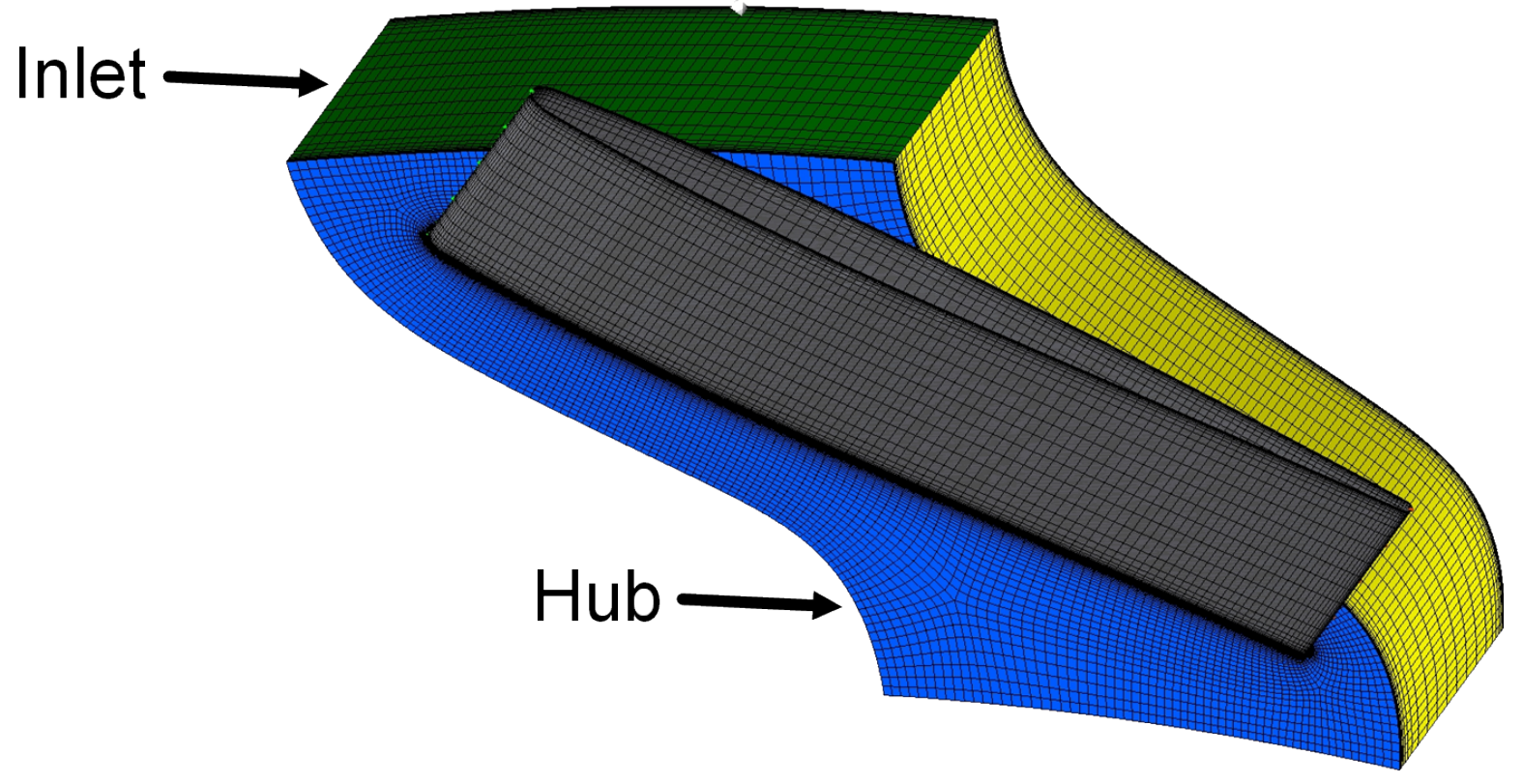

(a) Full domain.

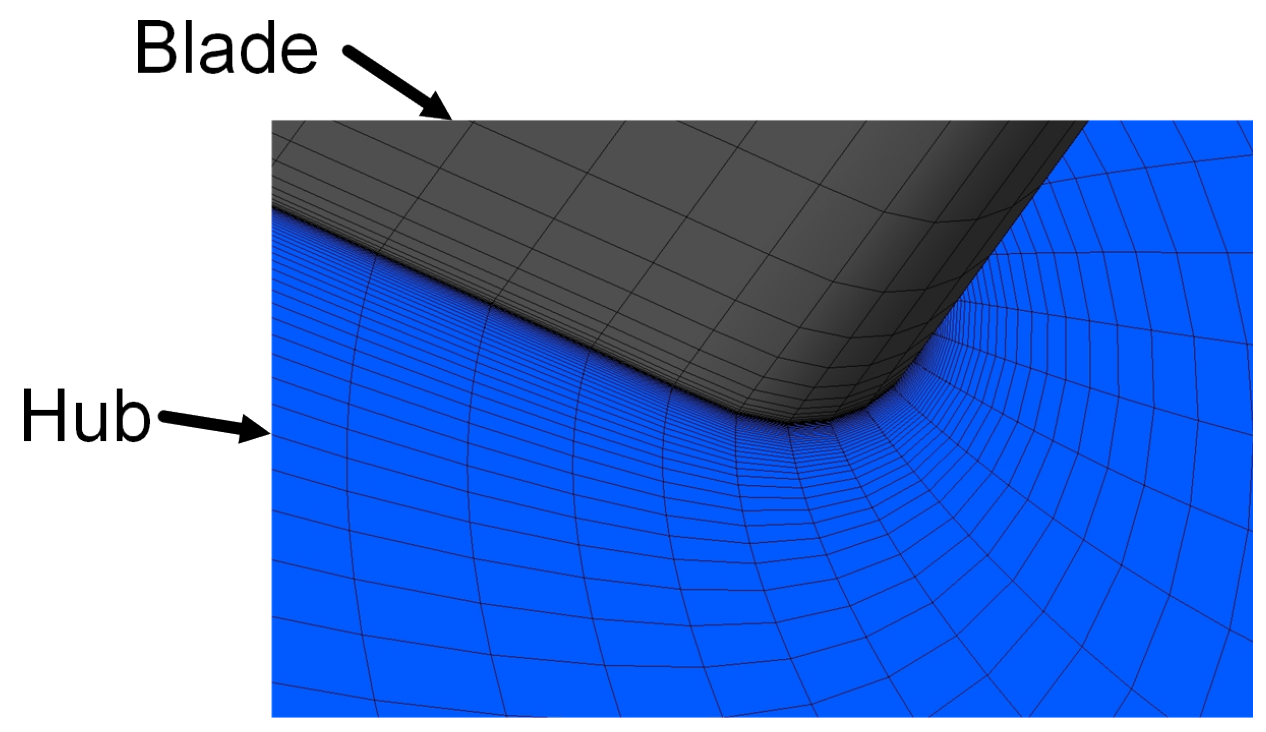

(b) Trailing edge hub location.

Figure C.2: Nozzle mesh detail. 


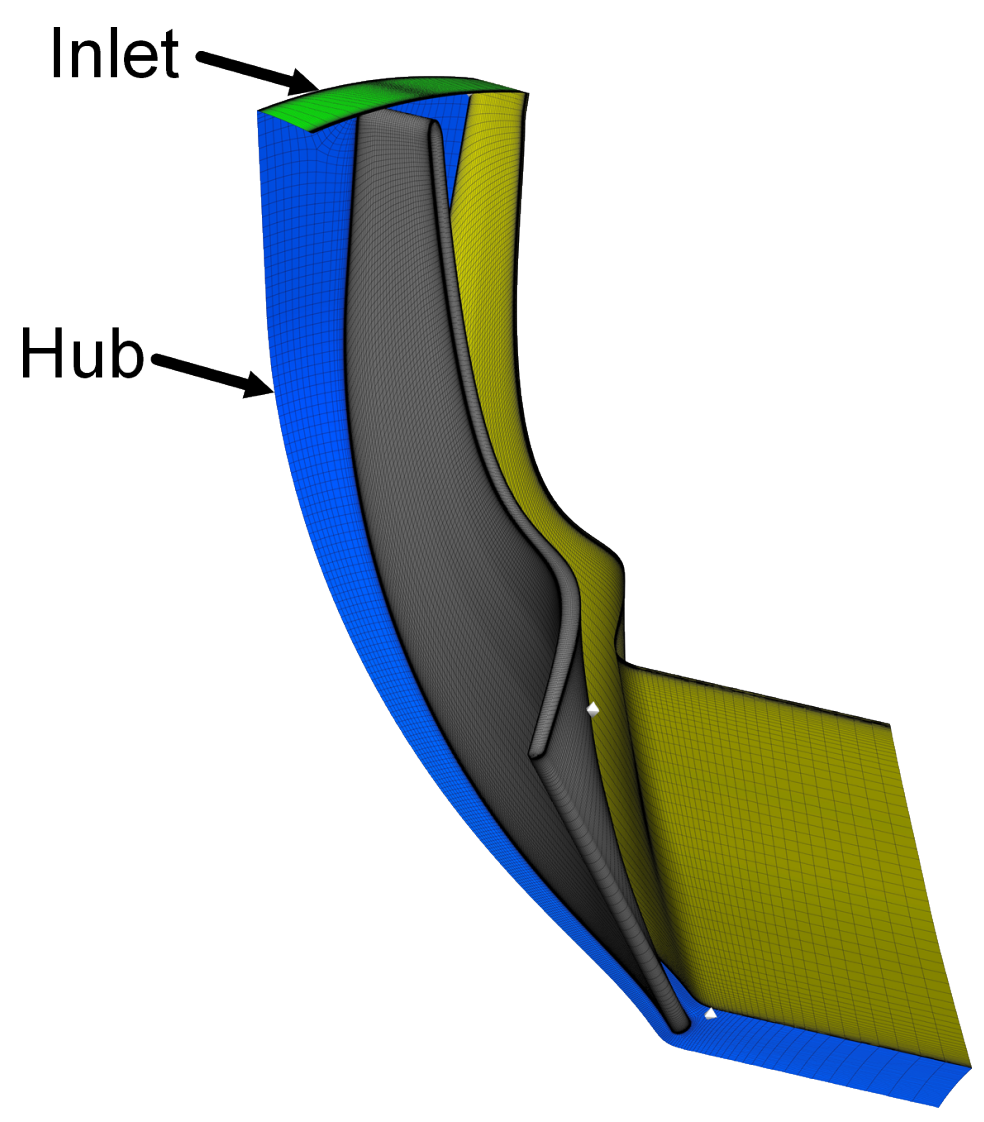

(a) Full domain.

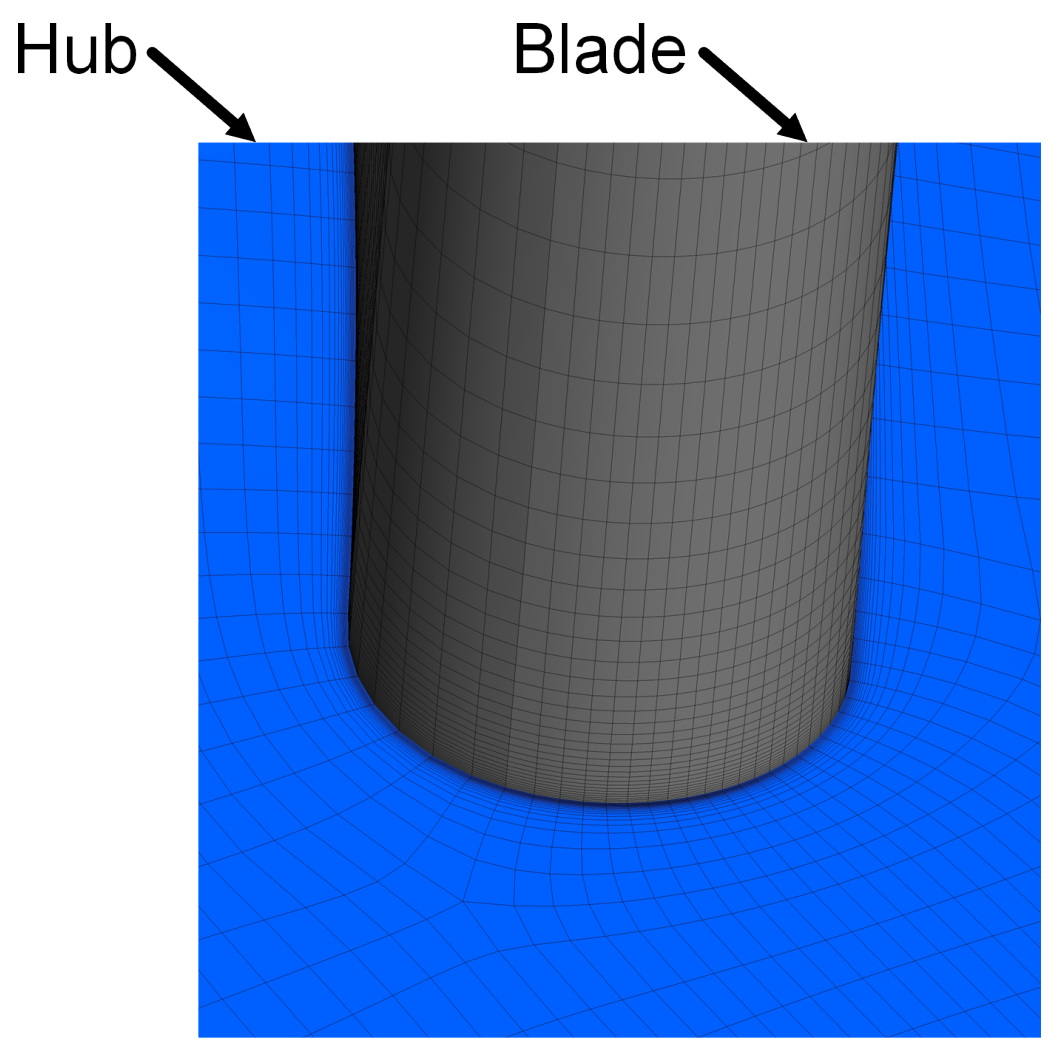

(b) Trailing edge hub location. 


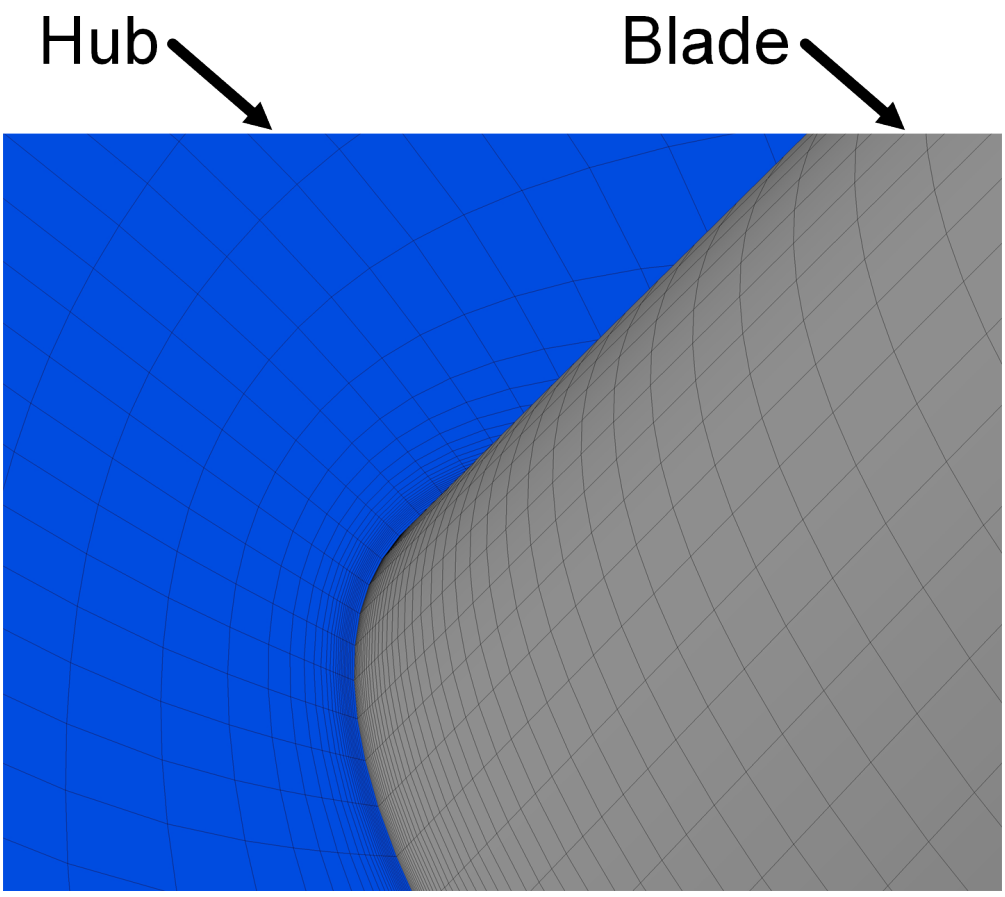

(c) Leading edge hub location.

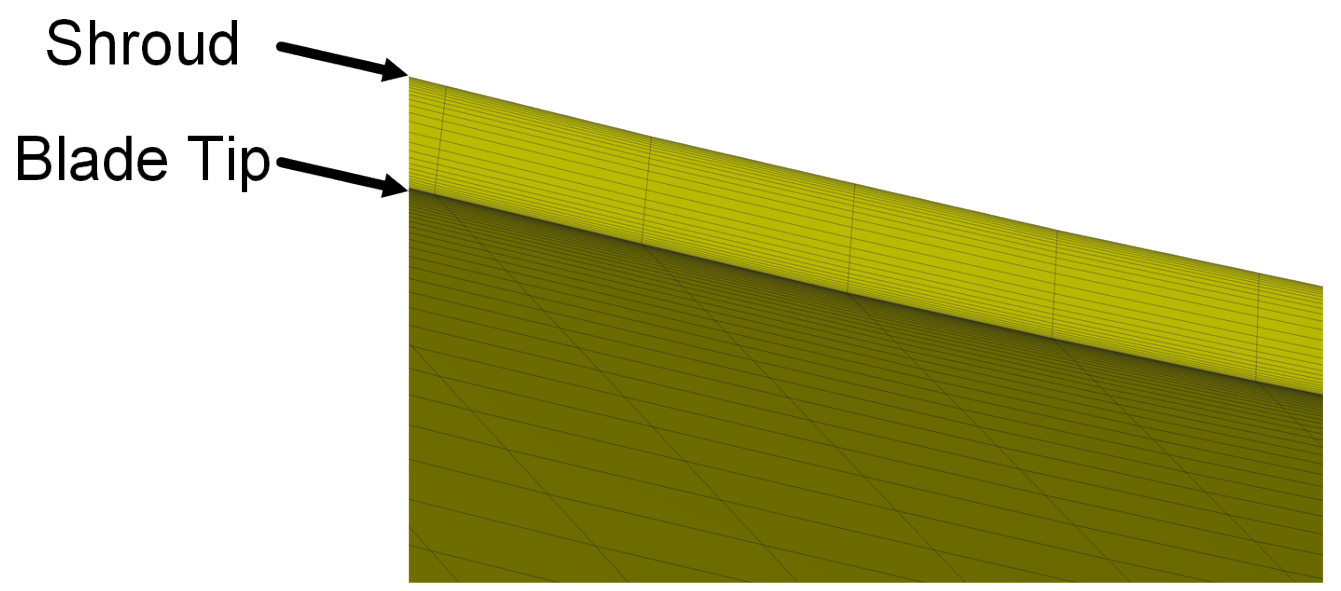

(d) Blade tip clearance location.

Figure C.3: Rotor mesh detail. 


\section{Appendix D}

\section{RGP Table Generation}

The RGP table generation script and resulting RGP table grids are presented in this Appendix.

\section{D.1 Script from ANSYS}

The following Python script was provided to the Gas Turbine research group at Carleton University by Robin Steed of ANSYS Inc. It is to be run within the terminal of the afd.bat Windows Batch File located in the ANSYS installation folder tree (for example, in C: $\backslash$ Program Files $\backslash$ ANSYS Student $\backslash v 182 \backslash A F D \backslash$ bin). The script creates RGP fluid property tables for $\mathrm{CO}_{2}$ in the temperature and pressure range specified by Tmin and Tmax, and Pmin and Pmax. This range should be large enough to account for all possible total and static conditions that may be seen in the CFD simulation. The tableResolution structure, resolution variable, and eps variable are used to specify the acceptable accuracy level in terms of the maximum error. For example, if resolution was specified as 'medium', then the specified maximum error, eps, would be $1 \times 10^{-4}$ according to the present script. The RGP is refined at every grid point until the acceptable error level is reached throughout when comparing property values interpolated from the RGP tables with values from REFPROP.

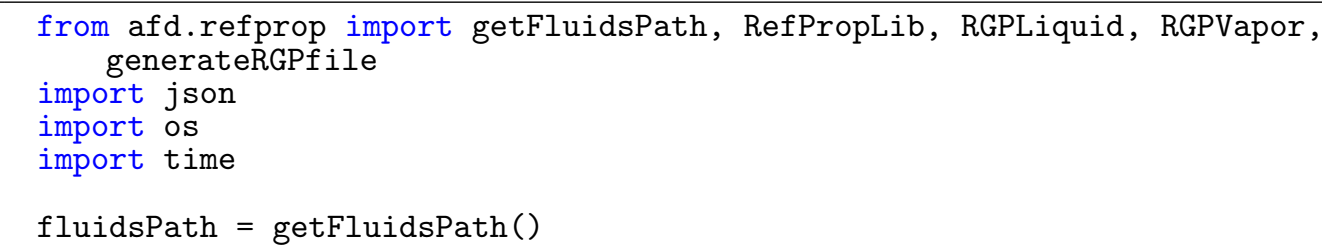




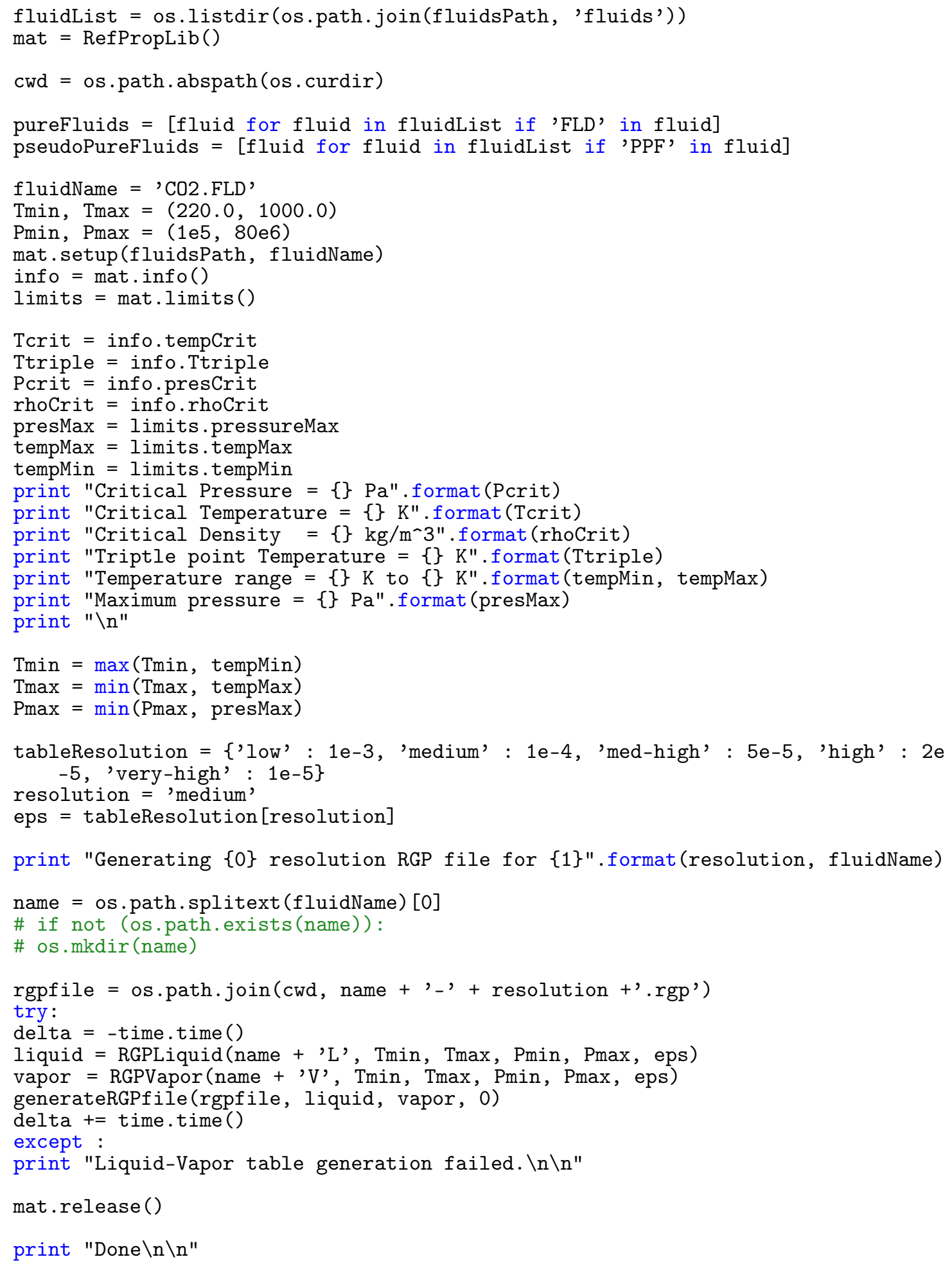




\section{D.2 RGP Tables}

Medium resolution tables were used in the present work. The operation of the turbine is in an area of the supercritical region where $\mathrm{CO}_{2}$ acts close to an ideal gas with a minimum compressibility factor around $Z=0.96$. This fact combined with property variation plots such as Figure 1.3 which indicate that the properties do not vary much at the states where the turbine operates led to the assumption that a maximum interpolation error of $1 \times 10^{-4}$ would be adequate for the present work.

The RGP tables used for the present work were built in the pressure and temperature range of $0.1 \mathrm{MPa}$ to $80 \mathrm{MPa}$ and $220 \mathrm{~K}$ to $1000 \mathrm{~K}$. This large was used to account for initial solution iteration where the flow calculations may reach those extremes, particularly in the tip clearance. The final RGP table grids used are shown in Figure D.1 through Figure D.9 where dependent variables associated with each table are indicated in Table D.1.

Table D.1: Dependent variable for the nine RGP tables.

\begin{tabular}{ll}
\hline \hline Table & Dependent Variable \\
\hline 1 & Specific enthalpy \\
2 & Speed of sound \\
3 & Specific volume \\
4 & Specific heat at constant volume \\
5 & Specific heat at constant pressure \\
6 & Partial derivative of pressure with \\
& respect to specific volume at \\
7 & constant temperature \\
8 & Specific entropy \\
9 & Dynamic viscosity \\
\hline \hline
\end{tabular}




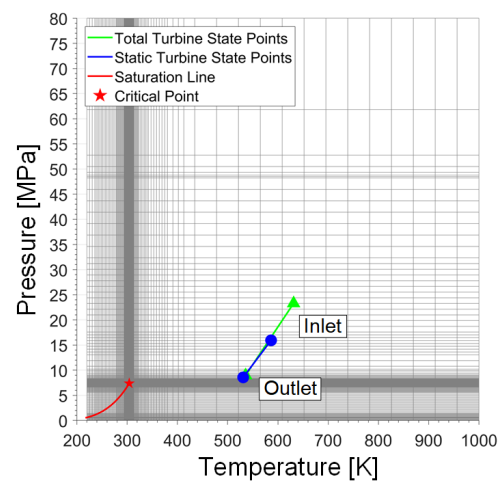

(a) Complete grid.

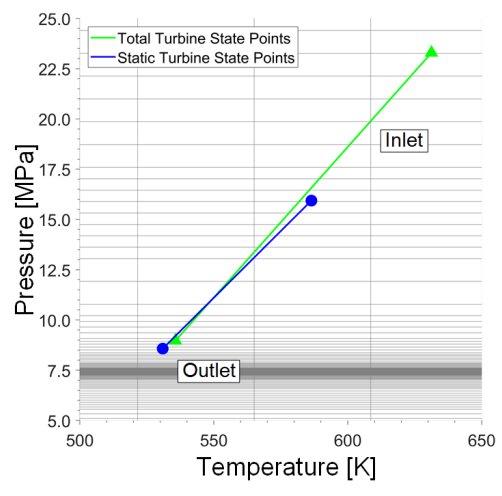

(b) Grid in turbine operating region.

Figure D.1: Pressure and temperature grids of Table 1 of RGP file used for the present work.

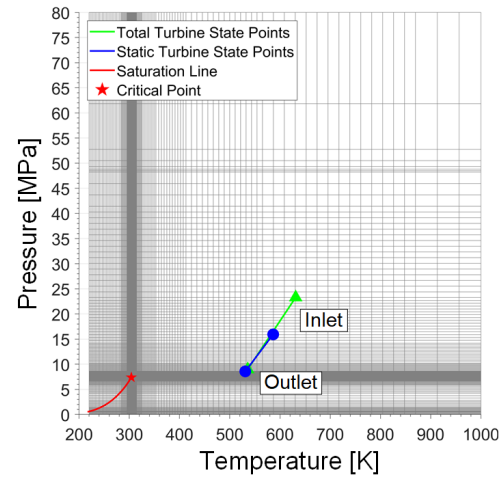

(a) Complete grid.

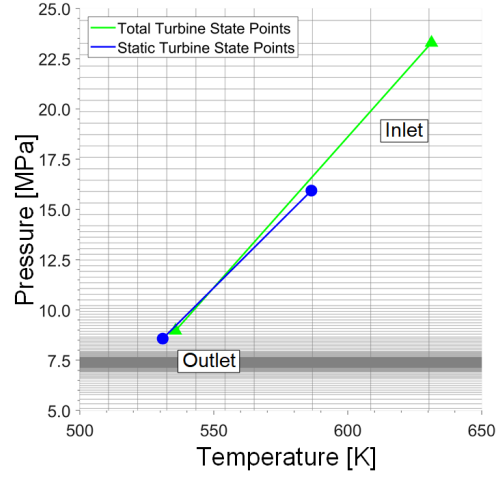

(b) Grid in turbine operating region.

Figure D.2: Pressure and temperature grids of Table 2 of RGP file used for the present work.

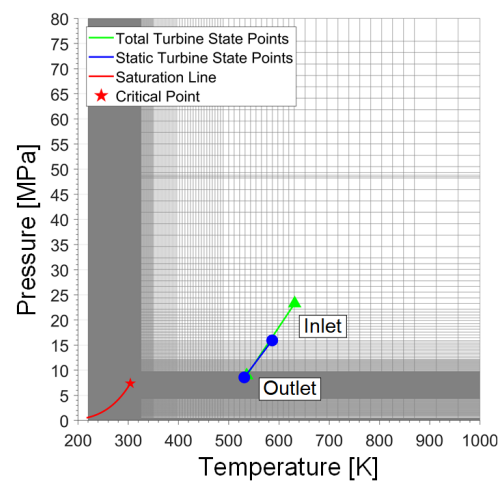

(a) Complete grid.

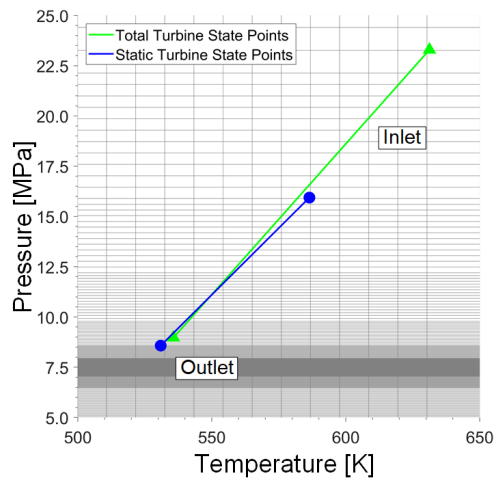

(b) Grid in turbine operating region.

Figure D.3: Pressure and temperature grids of Table 3 of RGP file used for the present work. 


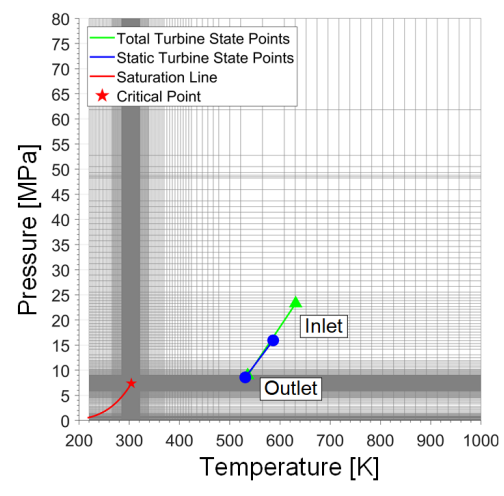

(a) Complete grid.

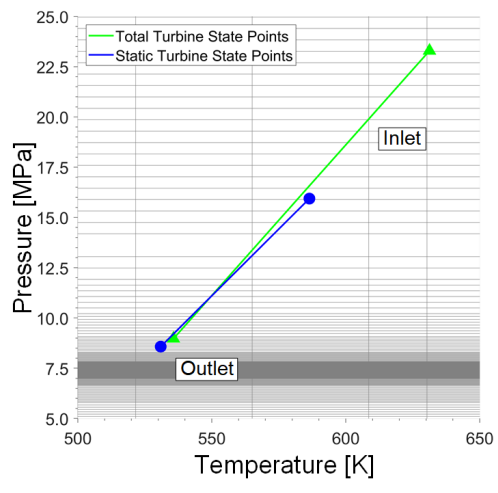

(b) Grid in turbine operating region.

Figure D.4: Pressure and temperature grids of Table 4 of RGP file used for the present work.

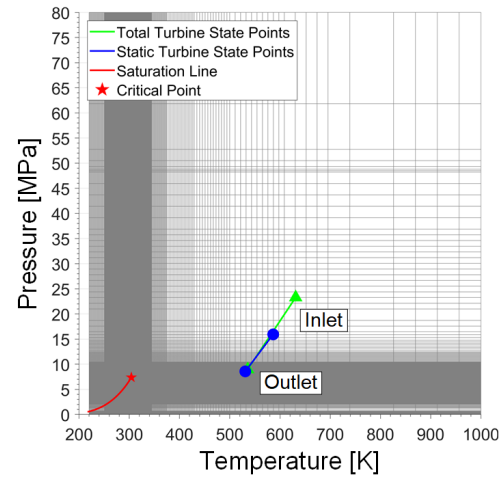

(a) Complete grid.

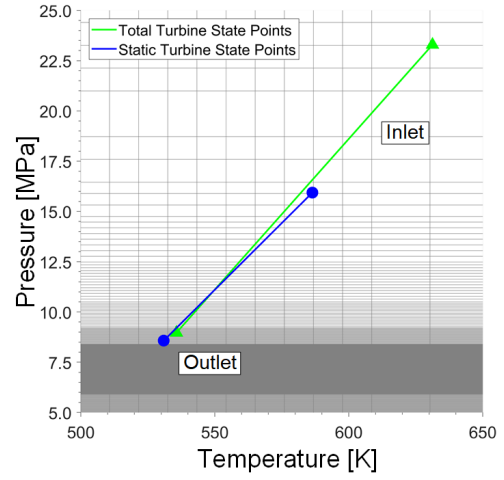

(b) Grid in turbine operating region.

Figure D.5: Pressure and temperature grids of Table 5 of RGP file used for the present work.

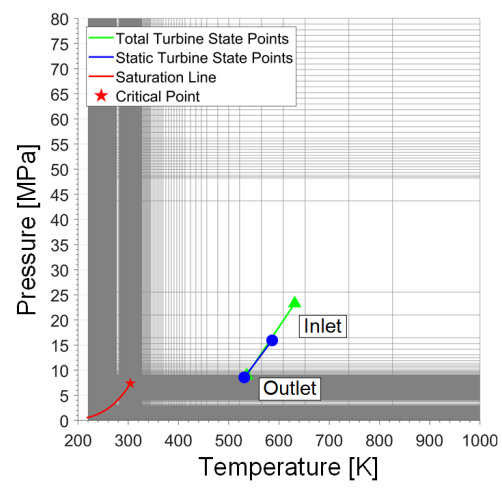

(a) Complete grid.

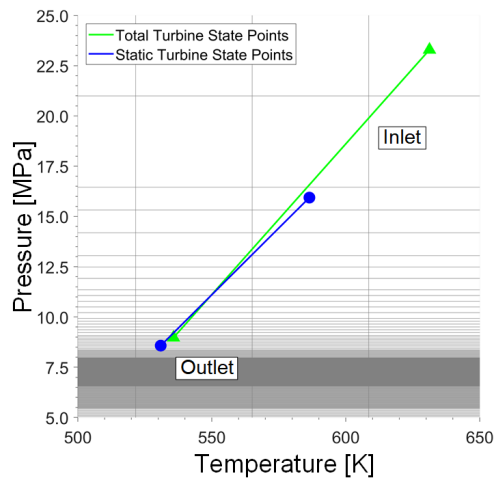

(b) Grid in turbine operating region.

Figure D.6: Pressure and temperature grids of Table 6 of RGP file used for the present work. 


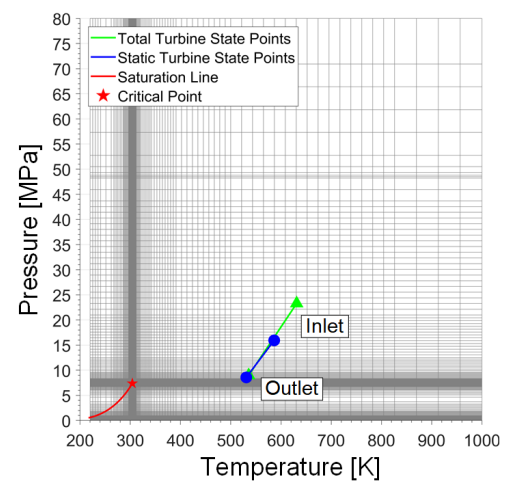

(a) Complete grid.

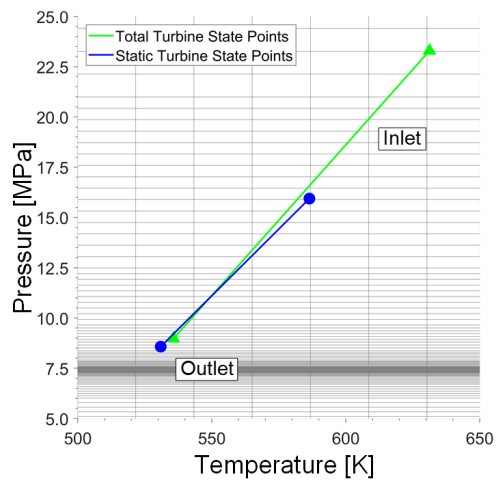

(b) Grid in turbine operating region.

Figure D.7: Pressure and temperature grids of Table 7 of RGP file used for the present work.

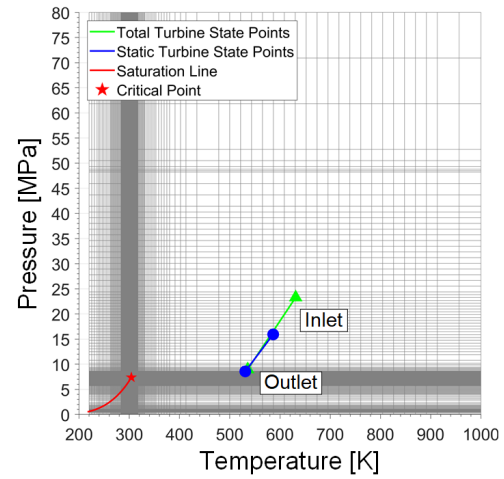

(a) Complete grid.

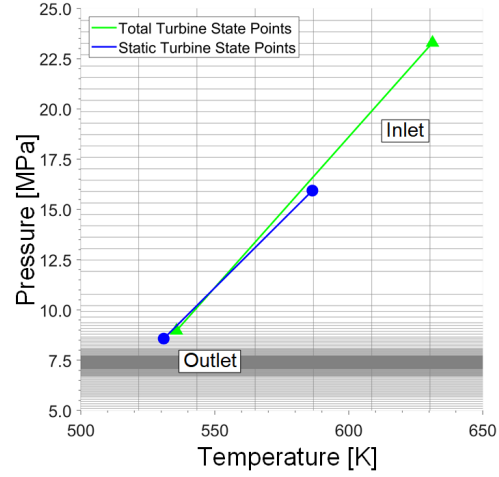

(b) Grid in turbine operating region.

Figure D.8: Pressure and temperature grids of Table 8 of RGP file used for the present work.

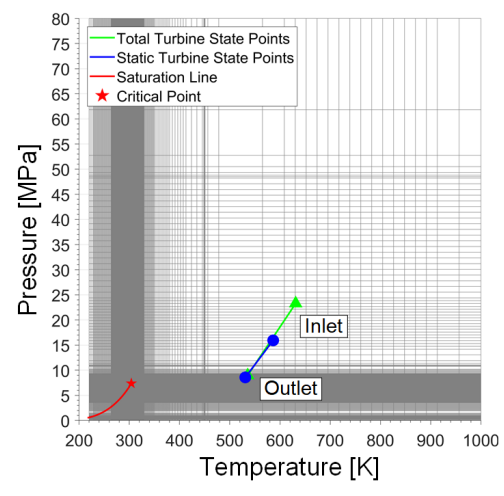

(a) Complete grid.

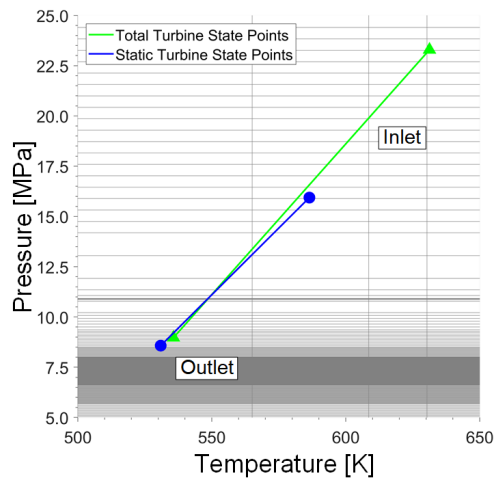

(b) Grid in turbine operating region.

Figure D.9: Pressure and temperature grids of Table 9 of RGP file used for the present work. 


\section{Appendix E}

\section{Performance Map Curve Fit Methods}

The curves fit to the turbine performance maps were based on methods used by White (2015). White (2015) based their Enthalpy Drop Parameter on the isentropic enthalpy drop, $\Delta h_{s s}=h_{01}-h_{5 s s}$, whereas it was based on the total enthalpy drop, $\Delta h_{0}=h_{01}-h_{05}$, in the present work. The relationship between the Flow Parameter and Enthalpy Drop Parameter was estimated by Equation E.1, where the coefficients $a, b$, and $c$ were determined by fitting the curve to the available CFD data. Figure E.1 shows the resulting curve fits. It was seen that the choking region (high Flow Parameter region) was not represented by the curve well because an asymptote below a Flow Parameter of 0.03 was suggested by the CFD data points but not by the curve. Therefore, a different curve fit method was investigated for the Flow Parameter to Enthalpy Drop Parameter relationship. It was found that the power law relation better represented the relationship between the Flow Parameter and the enthalpy ratio, $h_{01} / h_{05}$, as described by Equation E.2. The enthalpy ratio was converted into an enthalpy drop, $\Delta h_{0}$, with the known inlet enthalpy, $h_{01}$. The improved curve fit is shown in Figure E.2.

$$
\begin{aligned}
& \frac{\dot{m}}{\rho_{01} D_{4}^{2} a_{01}}=a\left(\frac{\Delta h_{0}}{a_{01}^{2}}\right)^{b}+c \\
& \frac{\dot{m}}{\rho_{01} D_{4}^{2} a_{01}}=a\left(\frac{h_{01}}{h_{05}}\right)^{b}+c
\end{aligned}
$$

White (2015) used a second order polynomial to relate the total-static efficiency to $\nu_{t s}=$ $U_{4} / \sqrt{2 \Delta h_{s s}}$. Whereas, in the present work, a second order polynomial was used to relate the 


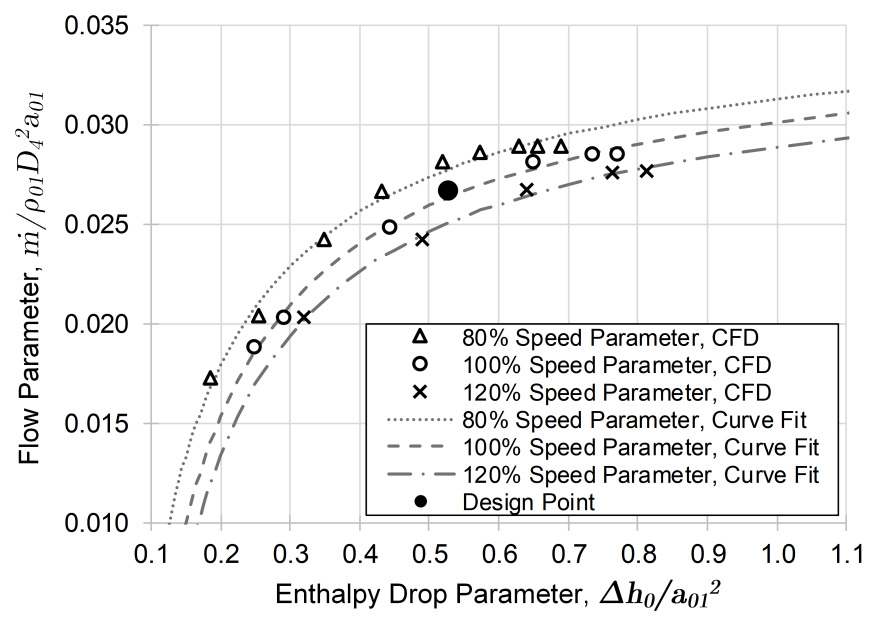

Figure E.1: Flow Parameter versus Enthalpy Drop Parameter for Speed Parameters of 80\%, $100 \%$, and $120 \%$ of the design speed with curve fit relationship from White (2015).

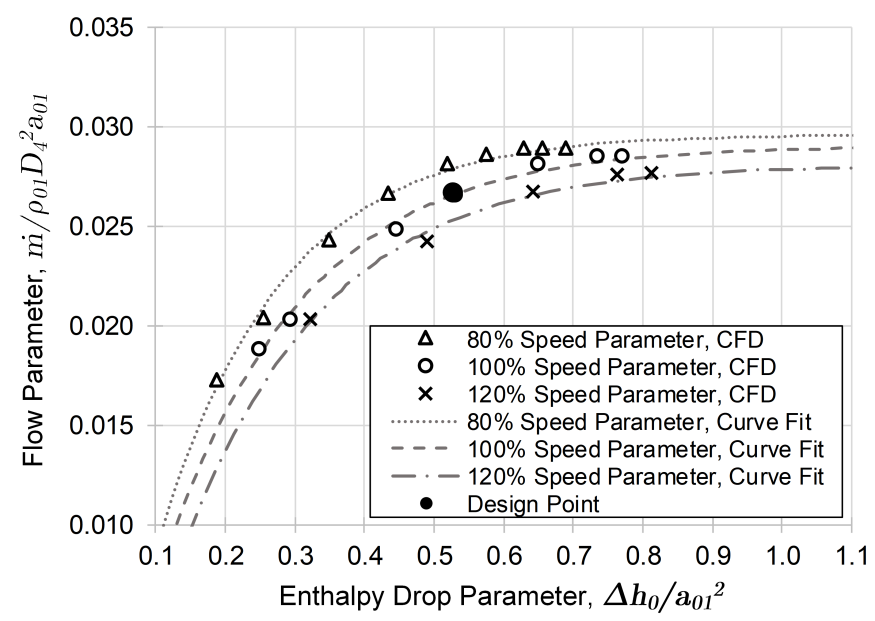

Figure E.2: Flow Parameter versus Enthalpy Drop Parameter for Speed Parameters of 80\%, $100 \%$, and $120 \%$ of the design speed with improved curve fit relationship.

total-total efficiency to $\nu_{t t}=U_{4} / \sqrt{2 \Delta h_{0}}$ (Equation E.3) which then implied the relationship between $\eta_{t t}$ and $\Delta h_{0}$. The resulting curves fit the data well as shown in Figure E.3. Again, the coefficients $d, e$, and $f$ were found by fitting the curve to the CFD data for a specific Speed Parameter.

$$
\eta_{t t}=d \nu_{t t}^{2}+e \nu_{t t}+f
$$




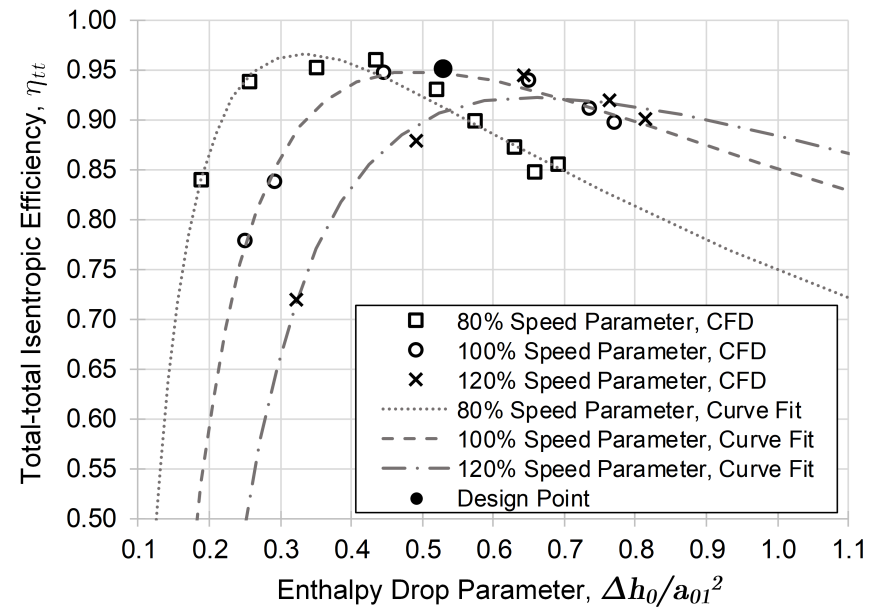

Figure E.3: Total-total Isentropic Efficiency versus Enthalpy Drop Parameter for Speed Parameters of $80 \%, 100 \%$, and $120 \%$ of the design speed. 